SACLAY-T06/001

$\mathrm{ECT}^{*}-06-01$

RBRC-581

\title{
Diffusive scaling and the high-energy limit of deep inelastic scattering in QCD at large $N_{c}$
}

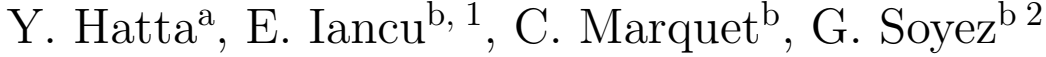 \\ and D.N. Triantafyllopoulos ${ }^{\mathrm{c}}$ \\ a RIKEN BNL Research Center, Brookhaven National Laboratory, Upton, NY 11973, USA \\ b Service de Physique Theorique de Saclay, CEA/DSM/SPhT, F-91191 Gif-sur-Yvette, France \\ c ECT*, Villa Tambosi, Strada delle Tabarelle 286, I-38050 Villazzano (TN), Italy
}

\begin{abstract}
Within the limits of the large $-N_{c}$ approximation (with $N_{c}$ the number of colors), we establish the high-energy behaviour of the diffractive and inclusive cross-sections for deep inelastic scattering at fixed impact parameter. We demonstrate that for sufficiently high energies and up to very large values of $Q^{2}$, well above the proton average saturation momentum $\left\langle Q_{s}^{2}\right\rangle$, the cross-sections are dominated by dense fluctuations in the target wavefunction, that is, by the relatively rare gluon configurations which are at saturation on the resolution scale $Q^{2}$ of the virtual photon. This has important physical consequences, like the emergence of a new, diffusive, scaling, which replaces the 'geometric scaling' property characteristic of the mean field approximation. To establish this, we shall rely on a dipole version of the Good-Walker formula for diffraction (that we shall derive here in the context of DIS), together with the high-energy estimates for the dipole scattering amplitudes which follow from the recently established evolution equations with Pomeron loops and include the relevant fluctuations. We also find that, as a consequence of fluctuations, the diffractive cross-section at high energy is dominated by the elastic scattering of the quarkantiquark component of the virtual photon, up to relatively large virtualities $Q^{2} \gg\left\langle Q_{s}^{2}\right\rangle$.
\end{abstract}

1 Membre du Centre National de la Recherche Scientifique (CNRS), France.

2 On leave from the Fundamental Theoretical Physics group of the University of Liège. 


\section{Contents}

1 Introduction $\quad 3$

2 A dipole picture for deep inelastic scattering at high energy 9

2.1 Dipole factorization for the diffractive cross-section 12

2.2 Justifying the dipole factorization 19

2.3 Evolution equations in the mean field approximation 26

3 Dipole amplitudes at high energy: Fluctuations \& Diffusive scaling 29

3.1 The event-by-event dipole amplitude 29

3.2 Front dispersion through fluctuations 33

$4 \quad$ Fluctuation effects on deep inelastic scattering at high energy 39

4.1 The general set-up 39

4.2 The $q \bar{q}$ component 43

4.3 The $q \bar{q} g$ component $\quad 54$

5 Conclusions $\quad 63$

A The virtual photon wavefunction 65

B The diffractive cross-section near $\beta=1 \quad 66$

$\begin{array}{ll}\text { References } & 67\end{array}$ 


\section{Introduction}

In this paper, we shall present the first analysis of the physical consequences of the fluctuations in the high-energy evolution in QCD for the phenomenology of lepton-hadron deep inelastic scattering (DIS). By "fluctuations" we mean in general the correlations in the gluon distribution of an energetic hadron (the target in DIS) which are generated through the radiative processes contributing to its evolution with increasing energy. More precisely, we shall be interested here in the gluon-number fluctuations, which have been recently shown [1-4] to play an essential role in the evolution of the elastic $S$-matrix for dipole-hadron scattering towards the unitarity limit. The "dipole" is a quark-antiquark pair in a colorless state, so like the $q \bar{q}$ excitation through which the virtual photon couples to the hadronic target in DIS. As demonstrated in Refs. [2-4], both the energy dependence of the characteristic scale for the onset of unitarization - the target saturation momentum $Q_{s}$ - and the functional form of the scattering amplitudes at high energy, are strongly modified, and to a large extent even determined, by such fluctuations. Motivated by these observations, and by the importance played by dipole configurations for the DIS processes at high energy, we shall provide a detailed analysis of the influence of gluon number fluctuations on the inclusive and diffractive DIS cross-sections. As we shall discover, the effects of fluctuations are in fact overwhelming: For sufficiently high energy, and within a wide kinematical range around the (average) saturation momentum, they wash out the corresponding predictions of the mean field approximation, and replace them with a new kind of universal behaviour, which is relatively simple.

A priori, one may expect the gluon-number fluctuations not to be important for the problem of the high-energy evolution, since the latter is characterized by a high-density environment (especially in the saturation regime at transverse momenta below $Q_{s}$ ), where the gluon occupation numbers are large. And as a matter of facts, such fluctuations have been left out by the modern approaches to non-linear evolution in QCD, namely, the Balitsky equations for the eikonal scattering amplitudes [5] and the (functional) JIMWLK equation [6-8] for the evolution of the 'color glass condensate' [8,9]. These formalisms do include non-trivial correlations - as obvious from the fact that they generate infinite hierarchies of equations - , but these are purely color correlations (like the exchange of a single gluon between two dipole amplitudes), and as such they die away when $N_{c} \rightarrow \infty$ (with $N_{c}$ the number of colors). In this limit, the Balitsky-JIMWLK hierarchy reduces to a single non-linear equation ${ }^{3}$ — the Balitsky-Kovchegov (BK) equation $[5,10]$-, which is often referred to as the mean field approximation (MFA). But in so far as the gluonnumber fluctuations of interest here are concerned, the Balitsky-JIMWLK formalism as a whole is a kind of MFA - the generalization of the BK equation to finite $N_{c}$.

Anticipated by early studies [11] (mostly numerical) within Mueller's "color dipole picture" [12], the importance of the gluon-number fluctuations for the high-energy evolution in QCD has been fully appreciated only recently, over the last couple of years [1-4]. Rare fluctuations associated with gluon splitting have been shown to control the formation of

3 More precisely, they reduce to a simplified hierarchy which is equivalent to the BK equation for uncorrelated initial conditions, and which does not generate new correlations in the course of the evolution; see the discussion in Ref. [4]. 
higher-point correlations in the dilute tail of the gluon distribution at relatively large transverse momenta (above $Q_{s}$ ). Once initiated through fluctuations, such correlations are rapidly amplified by the BFKL evolution [13], and then play a crucial role in the growth of the gluon distribution and its eventual saturation: they are at the origin of the saturation and unitarization effects included in the Balitsky-JIMWLK equations. Thus, while they are correctly describing the role of the higher-point correlations in providing saturation, the Balitsky-JIMWLK equations fail to also include the physical source for such correlations, namely, the gluon-number fluctuations in the dilute regime.

Soon after this failure has been first realized [4], new equations have been proposed [4, 14, 15] which correct the Balitsky-JIMWLK hierarchy by including the proper source terms (the relevant fluctuations) within the limits of the large $-N_{c}$ approximation. The latter is necessary since the dynamics leading to fluctuations in the dilute tail is described in the framework of the dipole picture $[12,16]$. There is currently an intense, ongoing, effort aiming at generalizing these equations to finite $N_{c}$ [17-24], but in spite of some interesting discoveries - like the recognition $[18,25]$ of a powerful self-duality of the high-energy evolution, and the construction of an effective Hamiltonian which is explicitly self-dual $[20,23]$ - , the general evolution equations valid for arbitrary $N_{c}$ are not yet known. This is the main reason for our restriction to the large $-N_{c}$ approximation in what follows.

The new equations in Refs. [4,14] form an infinite hierarchy for the $N$-dipole amplitudes $\left\langle T^{(N)}\right\rangle$ with $N \geq 1$ which describe the scattering between a system of $N$ color dipoles and an energetic hadronic target. For reasons to be briefly mentioned in Sect. 2.1, we shall refer to these equations as the 'Pomeron loop equations'. They are currently under active investigation [25-32], and although their general solutions are not known, a lot of information is already available about their asymptotic behaviour at high energy, via the correspondence $[3,4,33]$ between high-energy QCD and statistical physics (see the discussion below and also Sect. 3 for more details). This is the information that we shall use in our analysis of deep inelastic scattering throughout this paper.

Previous studies of DIS at high energy, or small "Bjorken- $x$ " [34-54] (see also the review papers [55-57]), have shown that diffractive DIS represents a privileged framework to study both gluon saturation - since the diffractive cross-section $\sigma_{\text {diff }}$ appears to be dominated by relatively large $q \bar{q}$ configurations, with size $r \sim 1 / Q_{s}\left(Q_{s}\right.$ is the target saturation momentum, and increases with the energy as $\left.Q_{s}^{2}(x) \sim 1 / x^{\lambda}\right)$ - and fluctuations since, as known since long $[58,59]$, the inelastic part of $\sigma_{\text {diff }}$ is a measure of the dispersion of the virtual photon wavefunction over its various Fock space components (like $q \bar{q}, q \bar{q} g$, etc.). Note, however, that the "fluctuations" which appear in the standard discussion of diffraction $[58,59]$ refer to the wavefunction of the projectile - in DIS, the virtual photon and its various hadronic excitations - , and not to that of the target (the proton, or more generally some hadron $h$ ). In such previous studies, the target has always been treated in the spirit of the mean field approximation, like an optical potential or some fixed gluon distribution, off which scatter the partonic components of the projectile. However, our main emphasis here will be precisely on the gluon-number fluctuations in the target wavefunction, which are the relevant fluctuations for a study of the high-energy limit, and which influence all the DIS observables, and not only those associated with diffraction. Indeed, as we shall see in Sect. 2, the calculation of both inclusive and diffractive cross-sections 


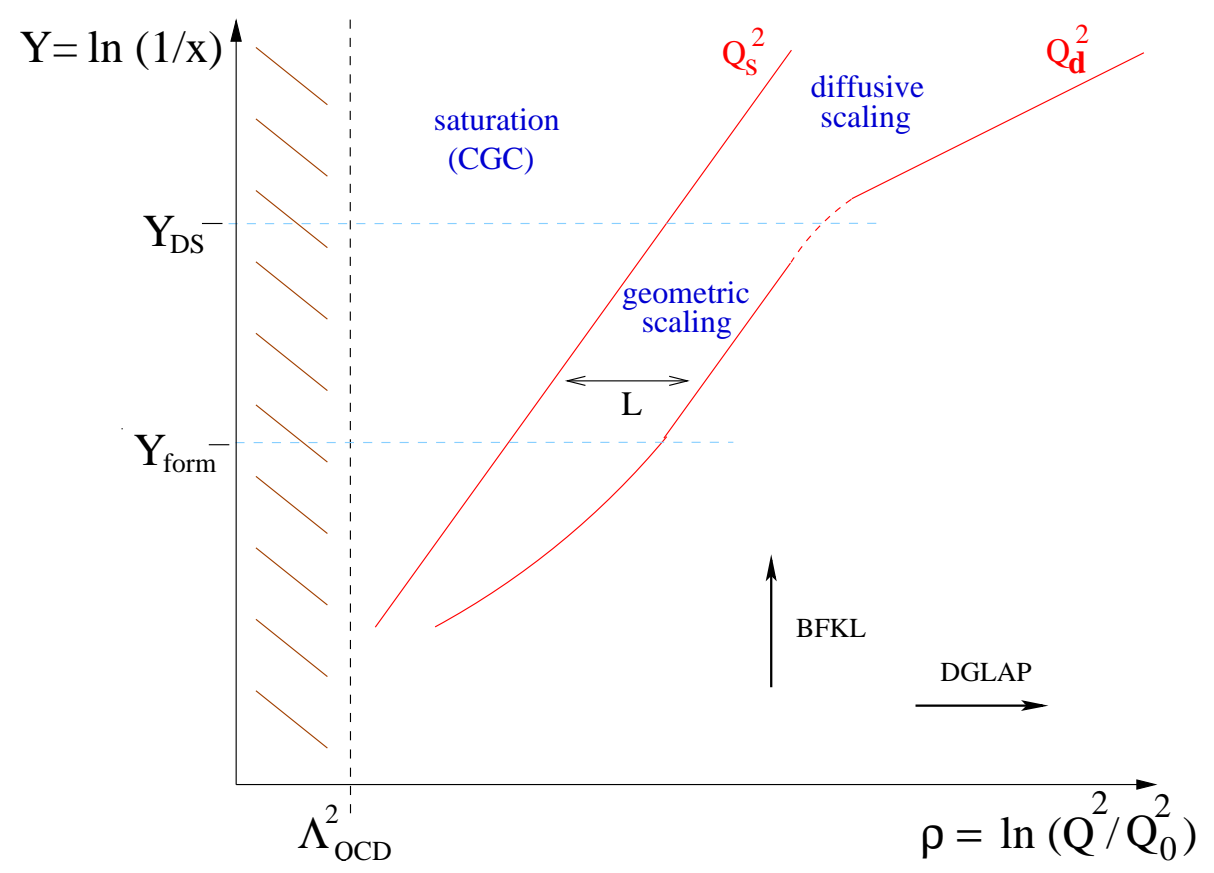

Fig. 1. "Phases" of the hadronic wavefunction in the kinematical plane $Y-\ln \left(Q^{2} / Q_{0}^{2}\right)$. When $Y \lesssim Y_{\text {DS }}$ the gluon distribution scales with momentum in a 'geometric' way, i.e. it is a function of $Q^{2} / Q_{s}^{2}$. When $Y \gtrsim Y_{\mathrm{DS}}$ it scales in a 'diffusive' way, i.e. it becomes a function of $\ln \left(Q^{2} / Q_{s}^{2}\right) / \sqrt{Y}$.

in DIS at high energy and large $N_{c}$ naturally involves the amplitudes for dipole-target scattering, as determined by the Pomeron loop equations alluded to above.

Let us clarify here what we shall mean by the 'high-energy regime of DIS' in what follows. This is the regime where, when increasing the energy (or decreasing $x$ ), the resolution scale $Q^{2}$ of the virtual photon is simultaneously increased (although not as fast as the total energy, of course !), in such a way that $Q^{2}$ remains comparable, or even larger, than the target saturation momentum $Q_{s}^{2}(x)$. Indeed, the strict high-energy limit, in which the energy is increased at a fixed value for $Q^{2}$ (and for a fixed impact parameter $b$ ), is less interesting, since conceptually clear: for sufficiently high energy, the cross-section reaches its unitarity (or 'black disk') limit, in which the projectile is completely absorbed by the target. (This behaviour will be manifest in the theoretical formalism that we shall use, which preserves unitarity.) On the other hand, the behaviour at relatively large $Q^{2}$, such that $Q^{2} \gg Q_{s}^{2}(x)$, is considerably more interesting, since in that region the scattering is weak, yet - as we shall discover - it is strongly influenced by the physics of saturation, within a large window in $Q^{2}$ whose width is increasing with $1 / x$. Moreover, with increasing energy, the effects of fluctuations in the evolution of the target gluon distribution become more and more pronounced, and the interplay between fluctuations and saturation leads to a rich structure in DIS at high energy and $Q^{2} \gtrsim Q_{s}^{2}(x)$, that we now explain.

To that aim, let us refer to Fig. 1, which represents the kinematical plane $Y-\rho$ (with $Y \equiv \ln (1 / x)$ and $\rho \equiv \ln \left(Q^{2} / Q_{0}^{2}\right)$, where $Q_{0}^{2}$ is a fixed reference scale) for DIS scattering, and also the physical phase-space for the QCD evolution of the hadronic target. Within the latter interpretation, $Y$ is the target rapidity and $Q^{2}$ can be assimilated to either the transverse momentum of a gluon inside the target $\left(k_{\perp} \sim Q\right)$, or the transverse size 
of a dipole within the projectile $(r \sim 1 / Q)$. The straight line denoted as " $Q_{s}^{2}$ " in Fig. 1 represents the average saturation line in the target: $\left\langle\rho_{s}\right\rangle_{Y} \equiv \ln \left(\left\langle Q_{s}^{2}\right\rangle / Q_{0}^{2}\right) \simeq \lambda Y$, with $\lambda$ the 'saturation exponent'. This is an 'average' since, in the presence of fluctuations, the saturation momentum becomes a random quantity: the stochastic evolution of the target gives rise to a statistical ensemble of gluon configurations, and the value of $Q_{s}^{2}$ can vary from one configuration to another. The dispersion $\sigma^{2} \equiv\left\langle\rho_{s}^{2}\right\rangle-\left\langle\rho_{s}\right\rangle^{2}$ which characterizes this variation will play a fundamental role in what follows. This stochastic process turns out to be a random walk in $\rho_{s} \equiv \ln \left(Q_{s}^{2} / Q_{0}^{2}\right)$, hence the dispersion rises linearly with $Y$ [3] $\sigma^{2}(Y) \simeq D_{\mathrm{fr}} Y$, with $D_{\mathrm{fr}}$ the 'front diffusion coefficient' (see Sect. 3). The second line in Fig. 1, denoted as " $Q_{d}^{2}$ ", will be shortly explained.

The most interesting region for us here is the scaling window in between these two lines. At lower values of $Q^{2}$, to the left of the saturation line, the gluons form a color glass condensate with large occupation numbers $\sim 1 / \alpha_{s}$, the dipole amplitude reaches the unitarity limit $\langle T\rangle=1$, and the mean field description is well justified. At much larger $Q^{2}$, on the right of the scaling region, one finds the standard perturbative regime, where the gluon system is very dilute, the dipole amplitude shows 'color transparency', $\langle T(r)\rangle \propto r^{2}$, and neither saturation, nor fluctuations, do play any role. But in the scaling region at intermediate $Q^{2}$, the gluon occupation numbers are relatively low and the scattering is weak, $\langle T\rangle \ll 1$, yet the dynamics is generally influenced by both saturation and fluctuations, in proportions which depend upon the actual value of $Y$.

The crucial scale for the present discussion is the rapidity $Y_{\mathrm{DS}}$ which separates between an intermediate energy regime at $Y<Y_{\mathrm{DS}}$, where $\sigma^{2} \ll 1$ and the mean-field picture roughly applies (with some important limitations, though, as we shall discuss in Sect. 3), and a high-energy regime at $Y>Y_{\mathrm{DS}}$, where $\sigma^{2} \gg 1$ and the target expectation values are dominated by rare fluctuations up to very large values of $\rho$. (Parametric estimates for $Y_{\mathrm{DS}}$ and the other scales which appear in Fig. 1 will be given in Sect. 3.)

Specifically, the lower part of the scaling region at $Y<Y_{\mathrm{DS}}$ is the window for geometric scaling [33, 60-63]. In that region, the dipole amplitude and the gluon occupation number 'scale' as functions of the dimensionless variable $Q^{2} /\left\langle Q_{s}^{2}\right\rangle$; e.g.,

$$
\langle T(r)\rangle_{Y} \approx\left(r^{2}\left\langle Q_{s}^{2}\right\rangle\right)^{\gamma_{0}} \equiv \exp \left[-\gamma_{0}\left(\rho-\left\langle\rho_{s}\right\rangle\right)\right], \quad \text { with } \quad \gamma_{0} \simeq 0.63
$$

This behaviour is particularly interesting as it may explain a similar scaling observed in the HERA data for DIS at $x \leq 0.01$ and up to rather large $Q^{2}\left(Q^{2} \lesssim 400 \mathrm{GeV}^{2}\right)[60]$.

But in the high-energy regime at $Y>Y_{\mathrm{DS}}$, the geometric scaling is progressively washed out by fluctuations [2] and eventually replaced (when $Y \gg Y_{\mathrm{DS}}$ ) by a new type of scaling $[3,4]$, for which we propose the name of diffusive scaling: the dipole amplitude scales as a function of the dimensionless variable $\left(\rho-\left\langle\rho_{s}\right\rangle\right) / \sigma$. This scaling extends up to very large values of $\rho$, namely, it holds so long as $\rho-\left\langle\rho_{s}\right\rangle \ll \sigma^{2}$. The borderline of this domain at $\rho_{d}(Y) \equiv\left\langle\rho_{s}\right\rangle+\sigma^{2} \simeq\left(\lambda+D_{\text {fr }}\right) Y$ represents the upper part of the line " $Q_{d}^{2}$ " in Fig. 1 . The functional form of the dipole amplitude is known within this entire domain [4] (see Sect. 3). In particular, deeply within the scaling window in Fig. 1, this is roughly a Gaussian:

$$
\langle T(\rho)\rangle_{Y} \approx \frac{\sigma}{\rho-\left\langle\rho_{s}\right\rangle} \exp \left\{-\frac{\left(\rho-\left\langle\rho_{s}\right\rangle\right)^{2}}{\sigma^{2}}\right\} \quad \text { for } \quad \sigma \ll \rho-\left\langle\rho_{s}\right\rangle \ll \sigma^{2} .
$$


The physics behind this asymptotic form is remarkably simple and universal (i.e., insensitive to both the initial condition at low energy and the details of the evolution with increasing energy). It relies on just two basic facts: (i) saturation exists - meaning that, in the event-by-event description, $T(\rho)=1$ for $\rho$ smaller than some $\rho_{s}$, and (ii) the saturation scale $\rho_{s}$ is a random variable distributed according to a Gaussian law, with expectation value $\left\langle\rho_{s}\right\rangle$ and dispersion $\sigma^{2}$ which both increase linearly with $Y$. Then, the scaling behaviour and the Gaussian shape manifest in Eq. (1.2) are a direct reflection of the corresponding properties of the probability distribution for $\rho_{s}$. These properties transmit so directly from the probability law to the average amplitude since, within the range indicated in Eq. (1.2), the expectation value $\langle T(\rho)\rangle$ is small, $\langle T(\rho)\rangle \ll 1$, yet it is dominated by gluon configurations which are at saturation at the scale $\rho$ of interest (i.e., for which $\rho_{s} \gtrsim \rho \gg\left\langle\rho_{s}\right\rangle$, and thus $T=1$ ) : Indeed, although relatively rare, such configurations yield contributions of order one, whereas the respective contributions of the typical configurations, for which $\rho_{s} \sim\left\langle\rho_{s}\right\rangle$, are exponentially suppressed (cf. Eq. (1.1)).

We thus arrive at the important conclusion that, even though the scattering is weak on the average, meaning that the target looks typically dilute when probed on the scale $r \sim$ $1 / Q$, the average amplitude is nevertheless controlled by "black spots" within the target wavefunction, i.e., by rare fluctuations with unusually large gluon density. At sufficiently high energy and up to extremely large values of $Q^{2}$ (much larger than $\left\langle Q_{s}^{2}\right\rangle$ ), the physical quantities are dominated by saturated gluon configurations. This conclusion will be further substantiated by our analysis of the DIS cross-sections at high energy.

Specifically, by using estimates like that in Eq. (1.2) for the dipole amplitudes $\left\langle T^{(N)}\right\rangle$ (the general formulae of this type will be presented in Sect. 3), together with appropriate, 'dipole', factorization fomualæ for the inclusive and the diffractive DIS cross-sections (that we shall discuss in Sect. 2), we shall analytically compute in Sect. 4 the dominant behaviour of the respective cross-sections in the high-energy regime - by which we shall more precisely mean the diffusive scaling regime at $\sigma^{2} \gg 1$ and $\rho-\left\langle\rho_{s}\right\rangle \ll \sigma^{2}$ (see Fig. $1)$. The main conclusion of this analysis can be concisely formulated as follows: All the physical properties (like the diffusive scaling or the Gaussian decrease in Eq. (1.2)) that have been previously noticed at the level of the dipole amplitudes translate literally to the DIS cross-sections. This is so since, as we shall demonstrate in Sect. 4, the various convolutions which relate the DIS cross-sections to the dipole amplitudes - namely, the convolutions with the BFKL kernel which enter the high-energy evolution of the projectile, and those with the wavefunction of the virtual photon - are dominated by small dipole sizes $r \sim 1 / Q$ (within the kinematic regime of interest here).

This last point is perhaps surprising, especially in relation with the diffractive sector, where the cross-section at large $Q^{2}\left(Q^{2} \gg Q_{s}^{2}\right)$ was known to be dominated by relatively large dipoles, with $r \sim 1 / Q_{s}[34,35,40,68]$. This standard picture is correct indeed, but at high energy it applies only in the event-by-event description, and not also on the average. Indeed, even for $Q^{2} \gg\left\langle Q_{s}^{2}\right\rangle$, the statistical ensemble representing the target does still contain gluon configurations ('black spots') for which $Q_{s}^{2} \sim Q^{2}$; although rare, such configurations will dominate the (inclusive and diffractive) cross-sections, because the photon wavefunction strongly favors the small dipole sizes $r \sim 1 / Q$.

Another surprise of our analysis is that, in the whole 'high-energy regime' defined as above 
- i.e., up to rather large values of $Q^{2}$, such that $Q^{2} \gg\left\langle Q_{s}^{2}\right\rangle-$, the diffractive crosssection is dominated by its elastic component, that is, by the elastic scattering between the onium as a whole and the hadronic target. (The 'onium' represents the wavefunction of the projectile at the time of scattering, as produced via the BFKL evolution of the original $q \bar{q}$ pair; see Sect. 2 for details.) Whereas expected in the 'black disk' regime at $Q^{2} \lesssim\left\langle Q_{s}^{2}\right\rangle$ $[56,58,59]$, this property may look surprising in the high $-Q^{2}$ regime at $Q^{2} \gg\left\langle Q_{s}^{2}\right\rangle$ (e.g., it contradicts the corresponding expectations of the MFA $[40,47,50]$ ), but it is in fact natural in view of our present wisdom: even for such high values of $Q^{2}$, the cross-section is dominated by 'black spots' which are already in the 'black disk' regime.

The dominance of the elastic scattering has interesting consequences for the dependence of the diffractive cross-section upon the rapidity gap $Y_{\text {gap }}$ : in the high-energy regime, the differential cross-section per unit rapidity gap is strongly peaked near $Y_{\text {gap }}=Y$. Alternatively — and this is the language that we shall prefer in this paper - the crosssection integrated over all the values of the rapidity gap $Y_{\text {gap }}$ from a minimal value $Y_{\text {gap }}^{\min }$ up to $Y$ is independent of the lower limit $Y_{\text {gap }}^{\min }$, so long as the latter is not too close to $Y$. This second language will be more convenient for us here since the approximations that we shall employ — a "leading logarithmic approximation" with respect to $\ln (1 / \beta) \equiv Y-Y_{\text {gap }}$ - will allow us to compute the integrated cross-section, but not also to control the details of the differential cross-section near $\beta=1$.

Let us mention here some limitations of our approach (besides the high-energy and large$N_{c}$ approximations already discussed). We shall not be able to describe the impact parameter dependence of the scattering amplitudes; that is, we shall follow their evolution with increasing $Y$ at a fixed impact parameter $b$. There are two reasons for that: First, the asymptotic solutions to the Pomeron loop equations are known only after a 'coarse-graining' in impact parameter space, which eliminates the dependence upon $b$ [4]. Second, more generally, we do not expect perturbation theory to properly describe the $b$-dependence of the scattering amplitudes, especially near the edge of the hadron, where the gluon density is low and the dynamics is sensitive to confinement. Because of that, in perturbation theory one can study the 'blackening' of the hadron disk, but not also the radial expansion of the 'black disk', i.e., the problem of the Froissart bound $[64,65]$.

Furthermore, in our study of diffraction, we shall not be able to describe processes with arbitrary large diffractive mass $M_{X}$, or arbitrary small values of $\beta$ (see kinematics in Fig. 2 ). Indeed, the formalism for diffraction that we shall develop in Sect. 2 can accommodate saturation effects in the wavefunction of the target (the hadron), but not also in that of the projectile (the virtual photon). This implies an upper limit on the rapidity $\ln (1 / \beta)$ of the projectile, which is however parametrically large: $\ln (1 / \beta) \ll\left(1 / \bar{\alpha}_{s}\right) \ln \left(1 / \alpha_{s}^{2}\right)$. Hence, in the case of diffraction, the 'high-energy limit' that we shall consider is the limit in which the total rapidity $Y$ and the rapidity gap $Y_{\text {gap }}$ can be arbitrarily large, but such that their difference $Y-Y_{\text {gap }}=\ln (1 / \beta)$ remains finite and constrained as indicated above.

This paper is organized as follows: In the first half of it, which covers Sects. 2 and 3, we shall develop the formalism which is required for a calculation of the DIS cross-sections in the high-energy regime and for large $N_{c}$, and in the second half (i.e., Sect. 4), we shall explicitly compute these cross-sections, and compare to the respective mean-field results. 
Specifically, in Sect. 2 we shall develop a factorization scheme for the diffractive dipolehadron scattering at high energy (the 'dipole' being, of course, the $q \bar{q}$ pair produced via the dissociation of the virtual photon in DIS). Our final formula turns out to be an interesting combination between the 'color dipole picture' by Mueller [12] and an early formula for hadron-hadron diffraction by Good and Walker [58]: Within the frame in which the target rapidity coincides with the minimal rapidity gap $Y_{\text {gap }}^{\min }$, the projectile is described as an onium - a collection of dipoles produced via the BFKL evolution of the original $q \bar{q}$ pair over a rapidity interval $Y-Y_{\text {gap }}^{\min }-$, and the diffractive crosssection is expressed as the onium expectation value of the elastic cross-sections for all the dipole configurations within the onium. Whereas the emergence of such a formula is not unexpected (as the dipoles fulfill the main requirement of the analysis in Ref. [58], namely, they are eigenstates of the $S$-matrix operator), our explicit derivation has the merit to clarify the importance of properly choosing the Lorentz frame in order for this formula to be valid. The relation to previous approaches $[36,46,50]$, in particular to a non-linear evolution equation proposed by Kovchegov and Levin [44], will be also discussed.

The aforementioned factorization formula involves the average dipole amplitudes $\left\langle T^{(N)}\right\rangle$ evaluated at the target rapidity $Y_{\text {gap }}$, which in our formalism can be arbitrarily large. In Sect. 3 we shall describe the calculation of these amplitudes in the high-energy regime, with the purpose of justifying expressions like Eq. (1.2) and completing the physical picture developed before, in relation with Fig. 1. The presentation in Sect. 3 is based on recent developments in Refs. [3,14], but it also contains some new elements and conceptual clarifications, like a parametric estimate for $Y_{\mathrm{DS}}$, the concept of diffusive scaling, and the recognition of an interesting 'rigidity' property for the average dipole amplitude in the presence of fluctuations.

Sect. 4 is our main section, in which we establish the dominant behaviour of the DIS crosssections in the high-energy regime. Remarkably, it turns out that this behaviour can be computed from the scattering amplitude $\langle T(r)\rangle_{Y}$ for a single dipole - the original $q \bar{q}$ fluctuation of the virtual photon. This is a priori clear for the inclusive cross-section, but it is true for the diffractive one (at high energy), because of the dominance of the elastic scattering, as aforementioned. Our calculation will be organized as follows: First, we shall consider the $q \bar{q}$ component alone and compute the respective contributions to $\sigma_{\text {tot }}$ and $\sigma_{\text {diff }}$. Then, we consider the additional contributions associated with the $q \bar{q} g$ component and demonstrate that, in the regime of diffusive scaling, the inelastic contribution is parametrically suppressed as compared to the elastic one. To better emphasize the effects of fluctuations, we shall compare our results to the corresponding predictions of the MFA.

Sect. 5 summarizes our results and presents our conclusions and some perspectives.

\section{A dipole picture for deep inelastic scattering at high energy}

As explained in the Introduction, we would like to provide a theoretical description for diffractive deep inelastic scattering at high energy and in the multi-color limit $N_{c} \rightarrow \infty$. By 'diffractive events' we shall understand the DIS events $\gamma^{*} h \rightarrow X p$ which contain an elastically scattered hadron $h$ in their final state together with a 'diffractive hadronic 


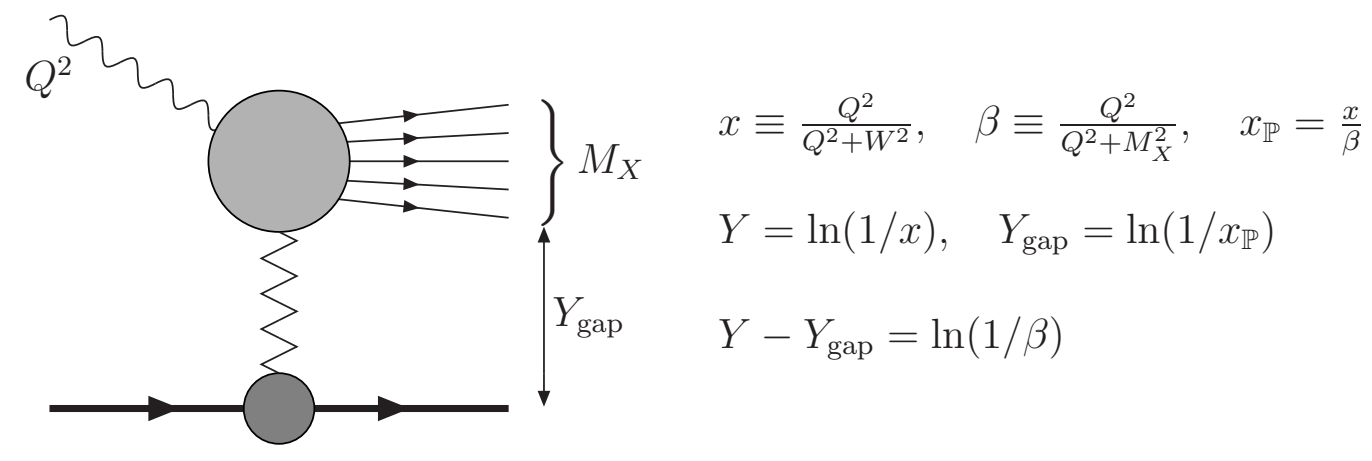

Fig. 2. Kinematics for diffractive DIS at high energy, or small Bjorken- $x$ : $Q^{2}$ is the virtuality of $\gamma^{*} ; W$ is the center-of-mass energy of the $\gamma^{*} h$ system (with $W^{2} \gg Q^{2}$ ); $M_{X}^{2}$ is the invariant mass squared of the diffractive system.

state' $X$ which is separated by a rapidity gap $Y_{\text {gap }}$ from the scattered hadron. (We use here standard notations in the context of DIS, as summarized in Fig. 2. See, e.g., Refs. $[55,56]$ for review papers on the phenomenology of diffraction and more details about the kinematics.) In the high energy regime at $W^{2} \gg Q^{2}, M_{X}^{2}$, the existence of a gap $Y_{\text {gap }} \simeq$ $\ln \left[W^{2} /\left(Q^{2}+M_{X}^{2}\right)\right]$ follows automatically from the condition that the proton undergoes elastic scattering together with the relevant kinematics. We shall be mainly interested here in the high-energy limit, defined as the limit $W^{2} \rightarrow \infty$ at fixed values for $Q^{2}$ and $M_{X}^{2}$. This means that the total rapidity difference $Y=\ln (1 / x)$ between the projectile $\left(\gamma^{*}\right)$ and the target $(h)$, as well as the rapidity gap $Y_{\text {gap }}=\ln \left(1 / x_{\mathbb{P}}\right)$ can increase arbitrarily large, but such that their difference $Y-Y_{\text {gap }}=\ln (1 / \beta)$ remains finite, and not too large (see Eq. (2.13) below for the precise condition). Note that our definitions for either diffraction or its high energy limit are not the most general possible ones (e.g., one could consider diffractive processes in which the target too breaks up after the collision), but they do cover interesting physical situations and, besides, they are constrained by the limitations of the subsequent theoretical developments.

As also mentioned in the Introduction, we shall directly compute the diffractive crosssection integrated over the rapidity gap from some minimal value $Y_{\text {gap }}^{\text {min }}$ up to $Y$. For convenience, from now on, we shall reserve the simpler notation $Y_{\text {gap }}$ for this minimal rapidity gap, as this quantity will appear very often, and we shall measure the actual rapidity by the corresponding $\beta$-variable, cf. Fig. 2 . We have, clearly, the following relation

$$
\frac{\mathrm{d} \sigma_{\text {diff }}^{\gamma}}{\mathrm{d}^{2} b}\left(Y, Y_{\text {gap }}, Q^{2}\right)=\frac{\mathrm{d} \sigma_{\text {el }}^{\gamma}}{\mathrm{d}^{2} b}\left(Y, Q^{2}\right)+\int_{0}^{Y-Y_{\text {gap }}} \mathrm{d} \ln (1 / \beta) \frac{\mathrm{d} \sigma_{\text {diff }}^{\gamma}}{\mathrm{d}^{2} b \mathrm{~d} \ln (1 / \beta)}\left(Y, \ln (1 / \beta), Q^{2}\right)
$$

between the integrated quantity in the l.h.s., that we shall explicitly compute in what follows, and the differential cross-section per unit rapidity - which appears under the integral sign in the r.h.s. and is the quantity usually considered in phenomenological 
studies of diffraction. As also indicated by the notations in Eq. (2.1), we shall consider cross-sections at fixed impact parameter $\boldsymbol{b}$. The first, "elastic", term in the r.h.s. of the above equation is simply the boundary term for the integration at $\beta=1$, or $Y_{\text {gap }}=Y$ :

$$
\frac{\mathrm{d} \sigma_{\mathrm{el}}^{\gamma}}{\mathrm{d}^{2} b}\left(Y, Q^{2}\right) \equiv \frac{\mathrm{d} \sigma_{\mathrm{diff}}^{\gamma}}{\mathrm{d}^{2} b}\left(Y, Y, Q^{2}\right)
$$

and the reason why we refer to it as "elastic" is because, within the "leading-logarithmic approximation' in $\ln (1 / \beta)$ that we shall use, it corresponds to the elastic scattering between the dissociation products of the virtual photon and the target (see Sect. 2.1 below for details and Appendix B for results going beyond the leading-logarithmic approximation alluded to above). Note however that the scattering is not elastic from the viewpoint of the virtual photon itself (see the discussion at the end of Sect. 2.1 and also Fig. 8).

Let us briefly describe here the physical picture underlying our subsequent developments. In a convenient 'dipole' frame in which the hadron $h$ carries most of the total energy, the scattering between the virtual photon $\gamma^{*}$ and the target proceeds as follows: Long before the scattering (say, at time $t_{0} \rightarrow-\infty$ ), the virtual photon dissociates into a quarkantiquark pair in a color singlet state (a 'color dipole'), which then evolves through soft gluon radiation until it meets the hadron (at time $t=0$ ) and scatters off the color fields therein. At high energy, the color dipole and the accompanying soft gluons are eigenstates of the $S$-matrix operator - the collision acts merely as a color rotation on these states - , and the original picture of diffraction by Good and Walker [58] can be taken over: The diffractive process $\gamma^{*} h \rightarrow X h$ consists in the elastic scattering between the various Fock space components of the projectile and the target. More precisely, we shall argue below that this simple picture holds only in a well-tuned frame, in which the rapidity $Y_{0}$ of the target coincides with the (minimal) rapidity gap $Y_{\text {gap }}$ in the final state ${ }^{4}$. Such a special choice of the frame is necessary in order to avoid the explicit treatment of the final state interactions, i.e., the gluon emissions and absorptions which take place in the wavefunction of the projectile after the time of scattering, and whose detailed description goes beyond the dipole picture.

As implicit in the above picture, we shall restrict ourselves to the leading logarithmic approximation at high energy, in which the evolution consists in the emission of 'small$x$ gluons', that is, gluons which carry only a small fraction $x \ll 1$ of the longitudinal momentum of their parent parton. So long as the energy is not too high, the evolution remains linear and is described by the BFKL formalism [13], which leads to a rapid growth of the gluon occupation numbers. But for sufficiently high energy, saturation effects which tame this growth start to be important $[9,68,69]$, and introduce non-linear effects in the evolution equations $[4-8,10,14]$. In the physical situations that we shall consider, the projectile will be always dilute and thus evolve linearly. On the other hand, we shall allow for arbitrarily high energies (and thus for saturation effects) on the side of the target.

The high-energy evolution will be further simplified through approximations valid at large $N_{c}$. On the projectile side, the combination of the BFKL evolution with the large-

${ }^{4}$ In practice, the identification of $Y_{0}$ with $Y_{\text {gap }}$ must hold within the accuracy of the leading logarithmic approximation: $Y_{0}$ and $Y_{\text {gap }}$ can differ by an amount $\mathrm{d} Y$ such that $\bar{\alpha}_{s} \mathrm{~d} Y \ll 1$. 
$N_{c}$ approximation leads to Mueller's 'color dipole picture' [12] : When $N_{c} \rightarrow \infty$, a gluon can be effectively replaced with a pointlike quark-antiquark pair in a color octet state, and a soft gluon emission from a color dipole can be described as the splitting of the original dipole into two new dipoles with a common leg. In this picture, the original $q \bar{q}$ pair produced by the dissociation of the virtual photon evolves through successive dipole splittings and becomes an onium - i.e., a collection of dipoles — at the time of scattering. Strictly speaking, the dipole picture provides only the norm of the onium wavefunction, that is, the probability distribution for the dipole configurations within the onium $[12,16]$. But, as we shall see, this knowledge is indeed sufficient to compute diffraction within the present formalism. On the target side, the large $-N_{c}$ approximation turns out to be essential for the successful construction of evolution equations for the amplitudes describing the scattering between the projectile dipoles and the target gluons $[4,14,15]$.

\subsection{Dipole factorization for the diffractive cross-section}

To develop our formalism, let us ignore for a while the electromagnetic process $\gamma^{*} \rightarrow q \bar{q}$ (at high energy, this process factorizes out $[70,71]$ and can be easily reintroduced later; see the beginning of Sect. 4.2), and focus on the onium-hadron $(\mathcal{O} h)$ scattering. The quantity that we would like to compute is the probability $P_{\text {diff }}\left(\boldsymbol{x}, \boldsymbol{y} ; Y, Y_{\text {gap }}\right)$ for the diffractive process $\mathcal{O} h \rightarrow X h$ in which the hadron $h$ undergoes elastic scattering while the onium $\mathcal{O}$ dissociates into some arbitrary hadronic state $X$ which is separated from the outgoing hadron by a rapidity gap equal to, or larger than, $Y_{\text {gap }}$ (with $Y_{\text {gap }} \leq Y$, of course). In this definition, $\boldsymbol{x}$ and $\boldsymbol{y}$ are the transverse coordinates of the quark and, respectively, the antiquark in the original $q \bar{q}$ pair, the one which evolves into the onium.

The probability $P_{\text {diff }}\left(\boldsymbol{x}, \boldsymbol{y} ; Y, Y_{\text {gap }}\right)$ is, of course, frame-independent, but in what follows we shall derive an explicit expression for it by working in the specific frame in which $Y_{\text {gap }}$ coincides with the rapidity $Y_{0}$ of the target (and where the projectile has therefore a rapidity $\left.{ }^{5} Y-Y_{0}=Y-Y_{\text {gap }}\right)$. To avoid redundant notations, we shall replace everywhere $Y_{\text {gap }} \rightarrow Y_{0}$ and write, e.g., $P_{\text {diff }}\left(\boldsymbol{x}, \boldsymbol{y} ; Y, Y_{0}\right)$. The final formula that we shall arrive at (see Sect. 2.2 for a derivation, and Figs. 3 and 4 for graphical representations) reads

$$
P_{\text {diff }}\left(\boldsymbol{x}, \boldsymbol{y} ; Y, Y_{0}\right)=\sum_{N=1}^{\infty} \int \mathrm{d} \Gamma_{N} P_{N}\left(\left\{\boldsymbol{z}_{i}\right\} ; Y-Y_{0}\right)\left|\langle 1-S(1) S(2) \cdots S(N)\rangle_{Y_{0}}\right|^{2}
$$

with notations to be explained now:

i) $P_{N}\left(\left\{\boldsymbol{z}_{i}\right\} ; Y-Y_{0}\right)$ is the probability density to produce a given configuration of $N$ dipoles after a rapidity evolution $Y-Y_{0}$ starting with an original dipole $(\boldsymbol{x}, \boldsymbol{y})$. The configuration is specified by $N-1$ transverse coordinates $\left\{\boldsymbol{z}_{i}\right\}=\left\{\boldsymbol{z}_{1}, \boldsymbol{z}_{2}, \ldots \boldsymbol{z}_{N-1}\right\}$, which physically represents the coordinates of the $N-1$ emitted gluons, and in terms of which the coordinates of the $N$ dipoles are $\left(\boldsymbol{z}_{0}, \boldsymbol{z}_{1}\right),\left(\boldsymbol{z}_{1}, \boldsymbol{z}_{2}\right), \ldots,\left(\boldsymbol{z}_{N-1}, \boldsymbol{z}_{N}\right)$, with $\boldsymbol{z}_{0} \equiv \boldsymbol{x}$ and

5 Within the leading-log approximation w.r.t. $\ln (1 / \beta)=Y-Y_{0}$, we can ignore the difference in rapidity between the quark and the antiquark components of the original $q \bar{q}$ pair and treat that pair as a 'particle' with unambiguous rapidity $Y-Y_{0}$. In Appendix B, we shall go beyond this leading-log approximation for the case where $\beta$ is close to one. 


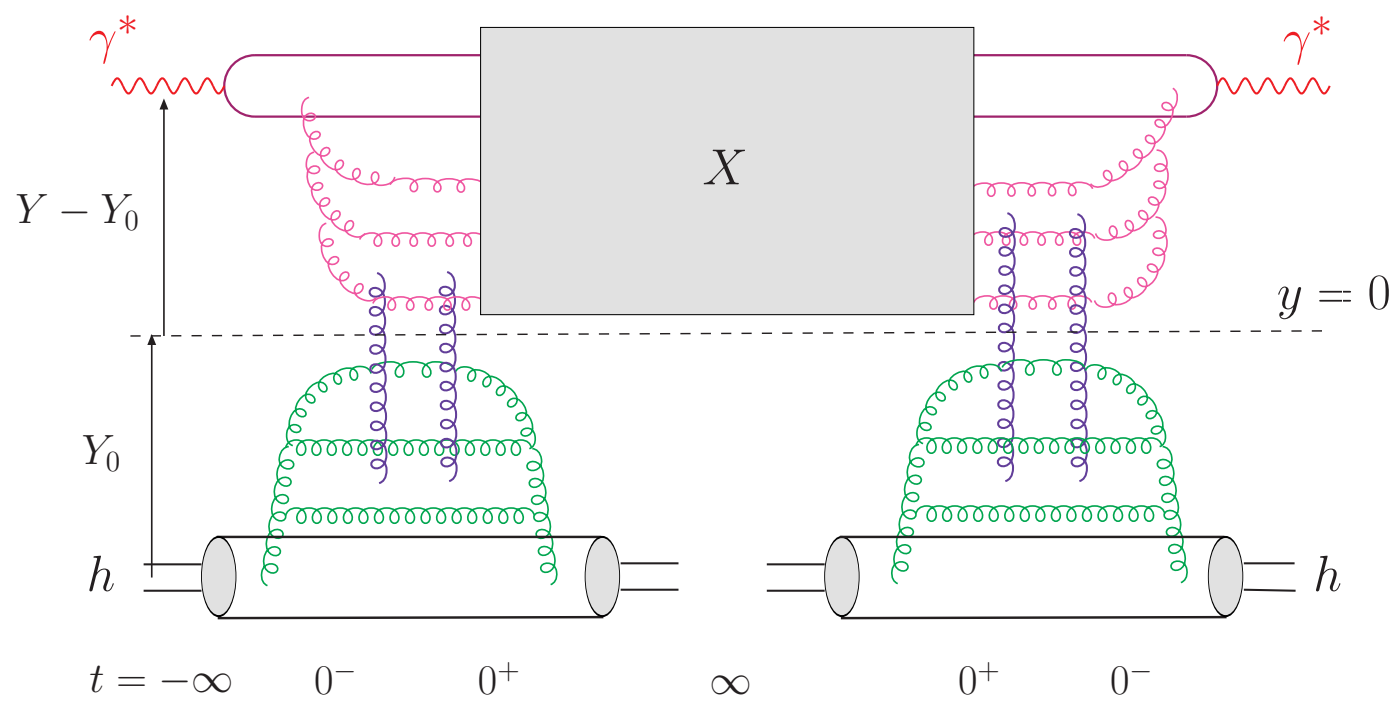

Fig. 3. Typical diagram contributing to the diffractive process $\gamma^{*} h \rightarrow X h$ in the frame where the target $Y_{0}$ coincides with the rapidity gap. For the projectile, we illustrate the gluon dynamics before and at the time of scattering. The final hadronic state $X$ can be formed with an arbitrary number of gluons produced via 'final state interactions' (see the discussion in Sect. 2.2). The gluons in the target recombine back before the final state, so that the hadron emerges intact from the collision. For simplicity, we exhibit only two-gluon exchanges.

$\boldsymbol{z}_{N} \equiv \boldsymbol{y}$. Also, $\sum_{N} \int \mathrm{d} \Gamma_{N}$ with $\mathrm{d} \Gamma_{N}=\mathrm{d}^{2} \boldsymbol{z}_{1} \mathrm{~d}^{2} \boldsymbol{z}_{2} \ldots \mathrm{d}^{2} \boldsymbol{z}_{N-1}$ represents the sum over all the configurations. The dipole probabilities are obtained by solving appropriate evolution equations (see below) with the following initial conditions :

$$
P_{1}(Y=0)=1, \quad P_{N>1}(Y=0)=0 .
$$

The evolution of the probabilities is such that the correct normalization condition

$$
\sum_{N=1}^{\infty} \int \mathrm{d} \Gamma_{N} P_{N}\left(\left\{\boldsymbol{z}_{i}\right\} ; Y\right)=1
$$

is satisfied at any $Y$.

ii) $S(i) \equiv S\left(\boldsymbol{z}_{i-1}, \boldsymbol{z}_{i}\right)$ is the $S$-matrix for the scattering between the $i$ th dipole in the projectile and a given configuration of color fields in the target. (Recall that, in the high-energy scattering, a dipole is an eigenstate of the interaction [70,71] and the internal configuration of the target is frozen during the duration of the collision; see also Eqs. (2.16)-(2.17) below.) We shall also need the scattering amplitude corresponding to a single dipole, defined as $T(i) \equiv 1-S(i)$. Then:

$$
\mathcal{S}_{N} \equiv \prod_{i=1}^{N} S(i) \quad \text { and } \quad \mathcal{A}_{N} \equiv 1-\mathcal{S}_{N}=1-\prod_{i=1}^{N}[1-T(i)]
$$

are respectively the $S$-matrix and the scattering amplitude for a given configuration of $N$ dipoles. The brackets in $\left\langle\mathcal{A}_{N}\right\rangle_{Y_{0}}$ denote the target average, that is, the average over the ensemble of color fields in the target. The target wavefunction, and thus the 


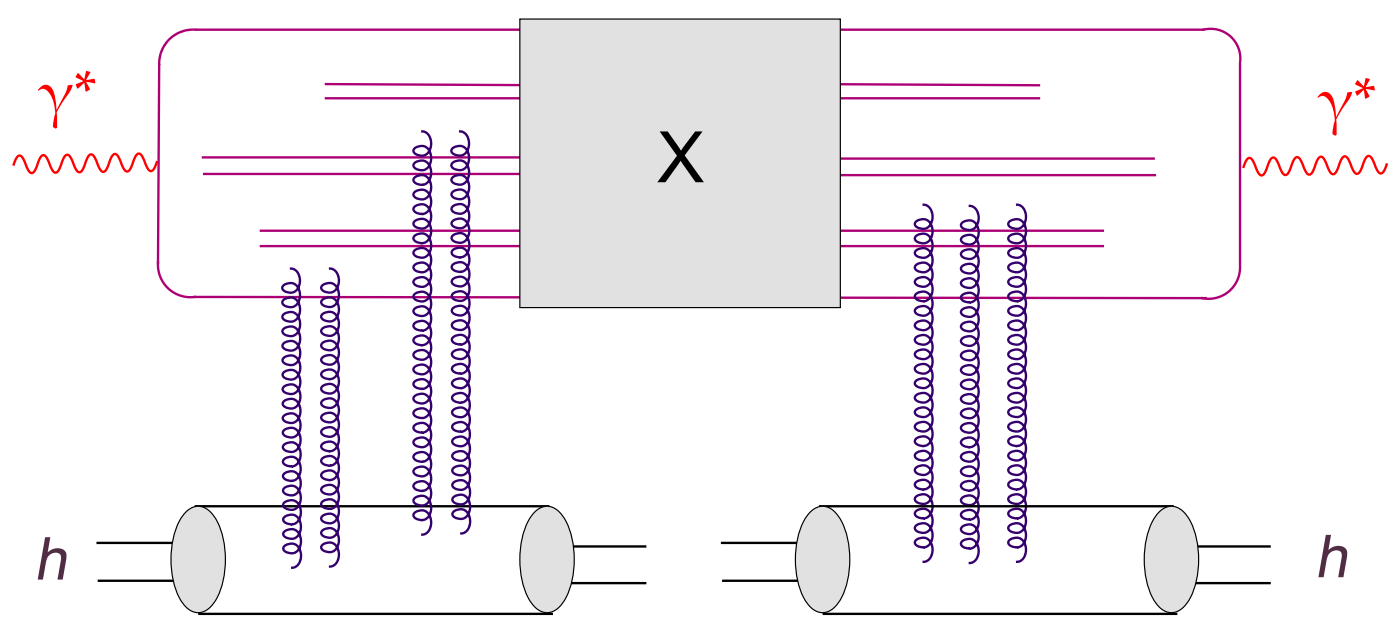

Fig. 4. The same as in Fig. 3 but in the large $-N_{c}$ limit. Each gluon in the wavefunction of the virtual photon has been replaced with a pointlike quark-antiquark pair in a color octet state. The gluons inside the target wavefunction are not shown explicitly anymore. The relative simplicity of the $q \bar{q}$ representation allows us to also exhibit some multiple gluon exchanges, corresponding to unitarity corrections.

corresponding expectation values, depend upon the rapidity interval $Y_{0}$ available for its internal evolution. Note that, in a given event (i.e., for a given configuration of the color fields in the target), the $N$ dipoles scatter independently from each other - the total $S$-matrix $\mathcal{S}_{N}$ is simply the product of $N$ factors corresponding to the individual dipoles - , but correlations are generally introduced by the average over the target (because the color fields there have non-trivial correlations). Thus, the expectation value $\left\langle\mathcal{S}_{N}\right\rangle_{Y_{0}}$ is not factorizing anymore. Such target correlations will play an important role in the subsequent discussion in this paper.

iii) The quantity $\left|\langle 1-S(1) S(2) \cdots S(N)\rangle_{Y_{0}}\right|^{2} \equiv\left|\left\langle\mathcal{A}_{N}\right\rangle_{Y_{0}}\right|^{2}$ is recognized as the probability for the elastic scattering between a given set of $N$ dipoles and the target. Thus, as anticipated, the diffractive probability (2.3) represents the projectile average of the elastic probabilities for all the possible (dipole) configurations in the projectile. Thus defined, the diffraction includes, but it does not reduces to, the truly elastic collision, in which the onium as a whole scatters elastically off the target. The difference appears because, whereas the individual dipoles (or gluons) are eigenstates of the interaction, this is not true also for their superposition (the onium), since the various states in this superposition interact differently with the target.

The elastic probability $P_{\mathrm{el}}$ for onium-target scattering is rather computed as

$$
P_{\mathrm{el}}(\boldsymbol{x}, \boldsymbol{y} ; Y)=|1-\mathcal{S}(\boldsymbol{x}, \boldsymbol{y} ; Y)|^{2} \equiv|\mathcal{A}(\boldsymbol{x}, \boldsymbol{y} ; Y)|^{2},
$$

where $\mathcal{S}(\boldsymbol{x}, \boldsymbol{y} ; Y)$ is the diagonal $S$-matrix element, $\mathcal{S} \equiv\left\langle\Psi_{\text {in }}|S| \Psi_{\text {in }}\right\rangle=\left\langle\Psi_{\text {in }} \mid \Psi_{\text {out }}\right\rangle$, which measures the overlap between the final state emerging from the collision and the initial state prior to it. Therefore, $P_{\text {survival }} \equiv|\mathcal{S}|^{2}$ is the probability that the original state (for the ensemble target + projectile) survives intact after the collision, whereas

$$
P_{\text {inel }}(\boldsymbol{x}, \boldsymbol{y} ; Y) \equiv 1-|\mathcal{S}(\boldsymbol{x}, \boldsymbol{y} ; Y)|^{2}
$$


is the probability for some inelastic process to occur. In what follows, the amplitude $\mathcal{A} \equiv 1-\mathcal{S}$ for the elastic scattering will be succinctly referred to as the forward amplitude. Within the present framework, this quantity can be computed as

$$
\mathcal{A}(\boldsymbol{x}, \boldsymbol{y} ; Y)=\sum_{N=1}^{\infty} \int \mathrm{d} \Gamma_{N} P_{N}\left(\left\{\boldsymbol{z}_{i}\right\} ; Y-Y_{0}\right)\langle 1-S(1) S(2) \cdots S(N)\rangle_{Y_{0}},
$$

which is perhaps a more familiar formula (various versions of it can be found in the literature $[10,30,72])$, and will be also derived below.

As indicated by its notation, the forward amplitude $\mathcal{A}$ is independent of the rapidity divider $Y_{0}$. This is a priori true on physical grounds - since, in the computation of $\mathcal{A}$, $Y_{0}$ plays no dynamical role, but merely specifies the Lorentz frame - and is also verified by our explicit formula (2.9), within its accuracy limits (see the discussion below). In particular, one can choose to compute $\mathcal{A}$ in the frame where $Y_{0} \simeq Y$. In that frame, the projectile is just an elementary dipole $(\boldsymbol{x}, \boldsymbol{y})$, without additional gluons, and therefore $\mathcal{A}(\boldsymbol{x}, \boldsymbol{y} ; Y)=1-\langle S(\boldsymbol{x}, \boldsymbol{y})\rangle_{Y} \equiv\langle T(\boldsymbol{x}, \boldsymbol{y})\rangle_{Y}$, in agreement with Eqs. (2.9) and (2.4). Similarly, the elastic probability (2.7) can be computed as the elastic scattering of the $q \bar{q}$ pair alone: according to Eqs. (2.3), (2.7), and (2.9) we can write

$$
P_{\mathrm{el}}(\boldsymbol{x}, \boldsymbol{y} ; Y)=\left|\langle T(\boldsymbol{x}, \boldsymbol{y})\rangle_{Y}\right|^{2}=P_{\mathrm{diff}}(\boldsymbol{x}, \boldsymbol{y} ; Y, Y)
$$

where the second equality reflects the physically obvious fact that an elastic scattering is the same as a diffractive event having $Y_{0}=Y$. This explains the identification performed in Eq. (2.2).

Via the optical theorem, the forward amplitude (2.9) also determines the total (or 'inclusive') cross- $^{-}$section ${ }^{6}$ :

$$
P_{\text {tot }}(\boldsymbol{x}, \boldsymbol{y} ; Y)=2 \operatorname{Re} \mathcal{A}(\boldsymbol{x}, \boldsymbol{y} ; Y)=P_{\text {el }}+P_{\text {inel }} .
$$

At this point, a word of caution is necessary, concerning a slight abuse in our terminology: The various "probabilities" introduced so far are strictly speaking differential cross-sections for onium-hadron scattering at fixed impact parameter; e.g.,

$$
\frac{\mathrm{d} \sigma_{\mathrm{tot}}}{\mathrm{d}^{2} b}(\boldsymbol{r}, \boldsymbol{b}, Y)=P_{\mathrm{tot}}(\boldsymbol{x}, \boldsymbol{y} ; Y), \quad \frac{\mathrm{d} \sigma_{\mathrm{diff}}}{\mathrm{d}^{2} b}\left(\boldsymbol{r}, \boldsymbol{b}, Y, Y_{0}\right)=P_{\mathrm{diff}}\left(\boldsymbol{x}, \boldsymbol{y} ; Y, Y_{0}\right),
$$

$(\boldsymbol{r} \equiv \boldsymbol{x}-\boldsymbol{y}$ and $\boldsymbol{b} \equiv(\boldsymbol{x}+\boldsymbol{y}) / 2$ are the transverse size and the impact parameter of the original $q \bar{q}$ pair). As such, these quantities are certainly proportional to the corresponding scattering probabilities, but they are not necessarily bound to be smaller than one. Rather, they are constrained by the unitarity of the $S$-matrix, which in the high energy regime (where the scattering amplitudes are predominantly real) requires $0 \leq\left\langle\mathcal{A}_{N}\right\rangle \leq 1$ for any $N$. This condition, together with the above formulæ, implies the standard inequality $P_{\text {diff }} \leq \frac{1}{2} P_{\text {tot }}[59]$, together with a series of upper bounds like $\mathcal{A} \leq 1, P_{\text {diff }} \leq 1$, and

${ }^{6}$ Note that our definitions for the scattering amplitudes $T$ and $\mathcal{A}$ differ by a factor of $i$ from the usual definitions in the textbooks. With our conventions, these amplitudes are predominantly real at high energy. 
$P_{\text {tot }} \leq 2$. Within the formalism that we shall use below to compute the dipole amplitudes, all these constraints are correctly respected, and the various upper bounds are saturated in the high-energy limit.

Before we turn to a derivation of Eqs. (2.3) and (2.9) in the next subsection, let us specify their range of validity in rapidity, and explain how to compute the dipole probabilities $P_{N}\left(Y-Y_{0}\right)$ and the target-averaged matrix elements $\left\langle\mathcal{A}_{N}\right\rangle_{Y_{0}}$ which enter these formulæ.

The rapidity $Y-Y_{0}$ of the projectile should be small enough for the saturation effects to remain negligible. This in turn requires $[12,16]$

$$
Y-Y_{0} \ll \frac{1}{\bar{\alpha}_{s}} \ln \frac{N_{c}^{2}}{\bar{\alpha}_{s}^{2}}
$$

where $\bar{\alpha}_{s} \equiv \alpha_{s} N_{c} / \pi$ should be treated as a fixed quantity in the large $-N_{c}$ limit. (Recall that the typical rapidity interval necessary for the emission of one small $-x$ gluon is $\left.\mathrm{d} Y \sim 1 / \bar{\alpha}_{s}.\right)$ For rapidities satisfying this constraint, the probabilities $P_{N}\left(Y-Y_{0}\right)$ can be obtained by solving a 'Master equation' [16], actually, a set of coupled, linear, equations for the evolution with $Y$, whose structure makes it clear that the dipole evolution in the dilute regime is a Markovian stochastic process (see also Refs. [4,73]). Alternatively, the generating functional for $P_{N}$, to be introduced in Eq. (2.35) below, obey the non-linear evolution equation (2.36), originally derived by Mueller [12]. For latter convenience, let us display here the expressions for $P_{N}$ generated after only one step in the evolution, that is, for $Y-Y_{0}=\mathrm{d} Y$ and to linear order in the small quantity $\bar{\alpha}_{s} \mathrm{~d} Y$ :

$$
\begin{aligned}
P_{1}(\mathrm{~d} Y) & =1-\mathrm{d} Y \frac{\bar{\alpha}_{s}}{2 \pi} \int \mathrm{d} \boldsymbol{z} \mathcal{M}(\boldsymbol{x}, \boldsymbol{y}, \boldsymbol{z}), \\
P_{2}(\boldsymbol{z} \mid \mathrm{d} Y) & =\mathrm{d} Y \frac{\bar{\alpha}_{s}}{2 \pi} \mathcal{M}(\boldsymbol{x}, \boldsymbol{y}, \boldsymbol{z}),
\end{aligned}
$$

and $P_{N}(\mathrm{~d} Y)=0$ for $N \geq 3$. In these equations,

$$
\mathcal{M}(\boldsymbol{x}, \boldsymbol{y}, \boldsymbol{z}) \equiv \frac{(\boldsymbol{x}-\boldsymbol{y})^{2}}{(\boldsymbol{x}-\boldsymbol{z})^{2}(\boldsymbol{y}-\boldsymbol{z})^{2}},
$$

is known as the 'dipole kernel' $[12]:\left(\bar{\alpha}_{s} / 2 \pi\right) \mathcal{M}(\boldsymbol{x}, \boldsymbol{y}, \boldsymbol{z})$ is the differential probability for an elementary dipole $(\boldsymbol{x}, \boldsymbol{y})$ to split into two dipoles $(\boldsymbol{x}, \boldsymbol{z})$ and $(\boldsymbol{z}, \boldsymbol{y})$ per unit rapidity. Note that the integral over $\boldsymbol{z}$ in the formula for $P_{1}$ has logarithmic singularities at $\boldsymbol{z}=\boldsymbol{x}$ and $\boldsymbol{z}=\boldsymbol{y}$. Such singularities are expected at the level of the dipole probabilities, but they cancel out in the calculation of physical quantities, as it can be checked on the examples of Eqs. (2.3) and (2.9).

Consider now the matrix elements for dipole-target scattering: At high energy, the $S_{-}$ matrix $S(\boldsymbol{x}, \boldsymbol{y})$ corresponding to a single dipole $(\boldsymbol{x}, \boldsymbol{y})$ can be computed in the eikonal approximation as

$$
S(\boldsymbol{x}, \boldsymbol{y})=\frac{1}{N_{c}} \operatorname{tr}\left(V^{\dagger}(\boldsymbol{x}) V(\boldsymbol{y})\right), \quad V^{\dagger}(\boldsymbol{x})=\mathrm{P} \exp \left\{i g \int d x^{-} A_{a}^{+}\left(x^{-}, \boldsymbol{x}\right) t^{a}\right\}
$$

where the Wilson lines $V^{\dagger}(\boldsymbol{x})$ and $V(\boldsymbol{y})$ represent the color rotations suffered by the quark and, respectively, the antiquark after their scattering off the color field $A_{a}^{+}$in the target. 
(The $t^{a}$ 's are the generators of the $\mathrm{SU}\left(N_{c}\right)$ algebra in the fundamental representation and the symbol $\mathrm{P}$ denotes path-ordering in $x^{-}$.) Note that, in our conventions, the projectile propagates in the negative $z$ (or positive $x^{-}$) direction, so it couples to the $A^{+}$component of the color field in the target. At high energy and in a suitable gauge, this is the only nontrivial component, and the average over the target wavefunction amounts to a functional average over $A^{+}$(the 'color glass' average $[8,9]$ ) :

$$
\langle S(\boldsymbol{x}, \boldsymbol{y})\rangle_{Y_{0}}=\int \mathrm{D}\left[A^{+}\right] W_{Y_{0}}\left[A^{+}\right] \frac{1}{N_{c}} \operatorname{tr}\left(V^{\dagger}(\boldsymbol{x}) V(\boldsymbol{y})\right)
$$

where the 'color glass weight function' $W_{Y_{0}}\left[A^{+}\right]$(a functional probability density) can be interpreted as the squared wavefunction of the target and depends upon the respective rapidity $Y_{0}$. The expectation value $\left\langle\mathcal{S}_{N}\right\rangle_{Y_{0}}$ for the scattering of $N$ dipoles is defined similarly. Note that, physically, the non-linear effects in $A^{+}$included via the Wilson lines in Eqs. (2.16)-(2.17) describe multiple scattering to all orders.

In the high-gluon density regime where the gluon-number fluctuations become negligible, the evolution of the weight function $W_{Y_{0}}$ with increasing $Y_{0}$ is described by the JIMWLK equation [6-8] - a functional, non-linear, equation of the Fokker-Plank type. Via equations like (2.17), the JIMWLK equation generates an hierarchy of ordinary evolution equations for the dipole amplitudes $\left\langle\mathcal{S}_{N}\right\rangle_{Y_{0}}$, originally derived by Balitsky [5]. The nonlinear effects encoded in the JIMWLK equation describe gluon saturation in the target wavefunction and translate into unitarity corrections in the Balitsky equations.

However, as pointed out in the Introduction, the gluon-number fluctuations missed by the Balitsky-JIMWLK equations are in fact essential for the physics at high energy, in that they represent the source for the higher-point correlations responsible for saturation. So far, the combined effects of fluctuations and saturation on the dipole scattering at high energy have been taken into account only in the large $-N_{c}$ limit $[2-4,15]$, where a new set of evolution equations has been derived $[4,14]$ — the 'Pomeron loop equations' alluded to in the Introduction (see also Refs. $[25,26,74]$ ). In what follows, we shall assume that the dipole amplitudes $\left\langle\mathcal{S}_{N}\right\rangle_{Y_{0}}$ obey these new equations, which within the limits of the large$N_{c}$ approximation are valid up to arbitrarily high energy. In addition to the standard BFKL terms [13], these equations include non-linear terms - which correspond to gluon saturation in the target and ensure the unitarization of the scattering amplitudes at high energy - and source terms - which correspond to dipole splitting in the dilute part of the target wavefunction and encode the relevant, gluon-number, fluctuations at large $N_{c}$. The perturbation theory for these equations can be organized in terms of "BFKL pomerons" (the Green's function for the BFKL equation [13]) which interact via "triple Pomeron vertices" [35,74,75] for Pomeron splitting and merging. Accordingly, the solutions to these equations naturally encompass the Pomeron loops. In Fig. 5, we illustrate some Pomeron loops effects in the calculation of the forward scattering amplitude (2.9).

To summarize, all the ingredients appearing in Eqs. (2.3) and (2.9) can be computed, at least in principle, by solving evolution equations which are explicitly known. These equations are valid for large $N_{c}$ and up to rapidities $Y$ and $Y_{0}$ which can be arbitrarily large, but such that their difference $Y-Y_{0}$ obeys the condition (2.13). Within this condition, the expression (2.9) for the forward amplitude is independent of the rapidity divider $Y_{0}$, 


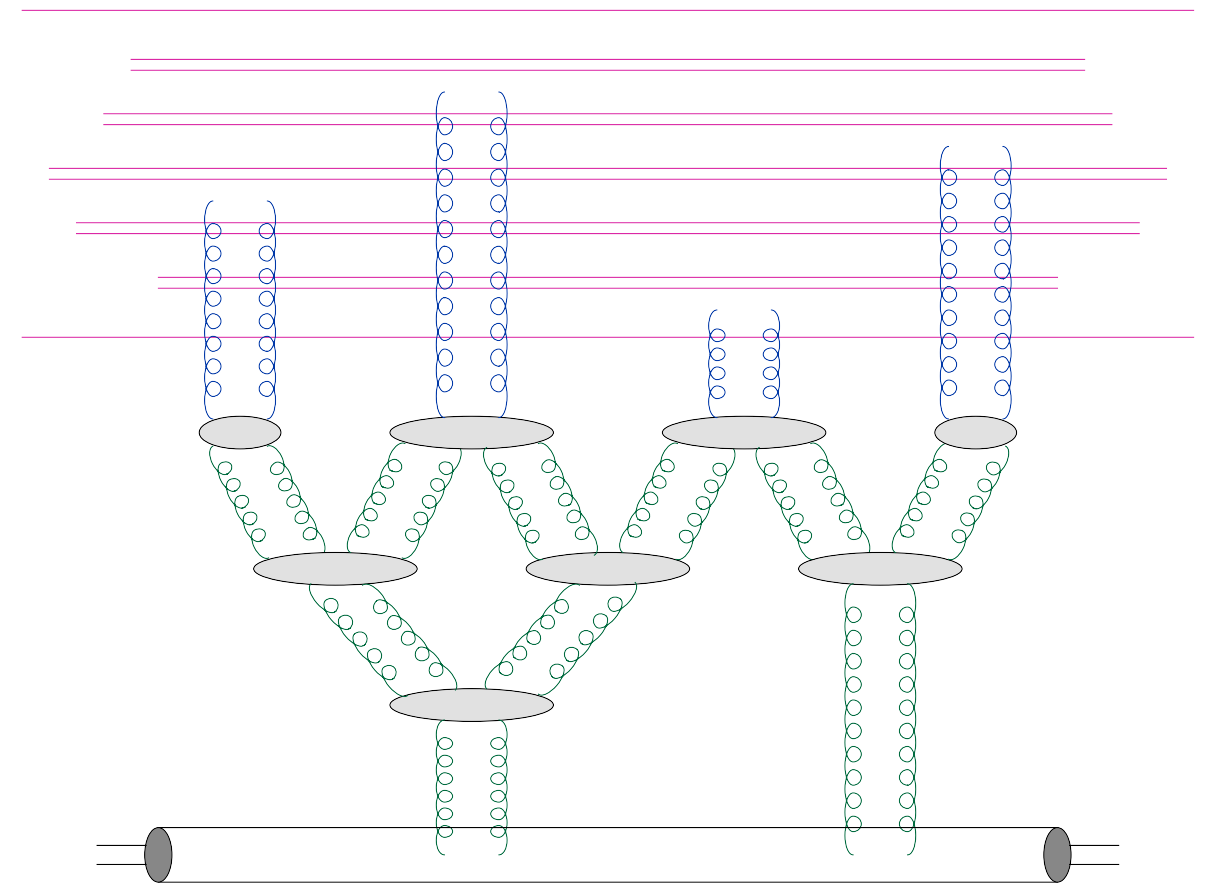

Fig. 5. Pomeron loops in the forward amplitude for onium-hadron scattering.

as it should. Indeed, it has been demonstrated in Refs. [30,72] that the $Y_{0}$-dependence in the r.h.s. of Eq. (2.9) cancels out exactly when using the Master equation for the dipole probabilities $P_{N}\left(Y-Y_{0}\right)$ together with the (large $-N_{c}$ version of the) Balitsky-JIMWLK equations for the dipole amplitudes $\left\langle\mathcal{S}_{N}\right\rangle_{Y_{0}}$. Now, as argued before, the correct equations at high energy are not the Balitsky-JIMWLK equations, but rather the Pomeron loop equations of Refs. [4,14]. The latter include the effects of gluon number fluctuations, which are additional sources of $Y_{0}$-dependence. In a more general calculation of $\mathcal{A}(\boldsymbol{x}, \boldsymbol{y} ; Y)$ which would be valid in any frame, such additional dependencies would be compensated by recombination effects in the wavefunction of the projectile. However, in any 'dipole frame' which satisfies the condition (2.13) such recombination effects are truly negligible, so Eq. (2.9) is indeed independent of $Y_{0}$, up to higher-order corrections ${ }^{7}$. Incidentally, the above argument also shows that our formula for the diffractive probability, Eq. (2.3), is not independent of $Y_{0}$, as expected on physical grounds.

For later convenience, let us also introduce here the inelastic diffraction, a process which, as shown in Ref. [59] in the context of hadron-hadron collisions, is a direct probe of 'parton' (here, dipole) fluctuations in the wavefunction of the projectile. The corresponding probability is obtained by simply subtracting out the (totally) elastic component (2.7) from the diffractive probability (2.3):

$$
P_{\text {diff }}^{\text {inel }}\left(\boldsymbol{x}, \boldsymbol{y} ; Y, Y_{0}\right)=P_{\text {diff }}\left(\boldsymbol{x}, \boldsymbol{y} ; Y, Y_{0}\right)-P_{\mathrm{el}}(\boldsymbol{x}, \boldsymbol{y} ; Y) .
$$

This formula takes on a particularly suggestive form after noticing that Eqs. (2.3) and (2.9) can be rewritten as (with simplified notations)

7 This does not mean that the physical consequences of the gluon-number fluctuations are also negligible (these will be discussed in the next sections). It is only the induced $Y_{0}$-dependence which is small indeed. 


$$
P_{\text {diff }}\left(\boldsymbol{x}, \boldsymbol{y} ; Y, Y_{0}\right)=\left\langle\left|\langle\mathcal{A}\rangle_{\text {target }}\right|^{2}\right\rangle_{\text {proj }}, \quad \mathcal{A}(\boldsymbol{x}, \boldsymbol{y} ; Y)=\left\langle\langle\mathcal{A}\rangle_{\text {target }}\right\rangle_{\text {proj }} .
$$

In these formulæ, the target-averaged amplitude $\mathcal{A}_{\text {target }} \equiv\langle\mathcal{A}\rangle_{\text {target }}$ is still an 'operator' from the point of view of the projectile, in the sense of depending upon a fixed configuration of dipoles. Also, at high energy, the amplitude is predominantly real, so one can ignore the modulus sign in the previous equations. We thus finally arrive at [59]

$$
P_{\text {diff }}^{\text {inel }}\left(\boldsymbol{x}, \boldsymbol{y} ; Y, Y_{0}\right)=\left\langle\mathcal{A}_{\text {target }}^{2}\right\rangle_{\text {proj }}-\left\langle\mathcal{A}_{\text {target }}\right\rangle_{\text {proj }}^{2},
$$

which clearly exhibits the fact that $P_{\text {diff }}^{\text {inel }}$ is a measure of the dispersion of the dipole distribution within the wavefunction of the projectile. The dipole picture that we employ here provides an explicit realization for the projectile, and thus allows one to compute this dispersion and any other quantity pertinent to the distribution of dipoles. However, our main emphasis in what follows will be not on the effects of fluctuations in the projectile (these are already well understood within the dipole picture; see, e.g., Refs. [4,11,12, $16,72,73]$ ), but rather on the physical consequences of the gluon number fluctuations in the target, as encoded in the evolution equations with Pomeron loops [4,14]. These fluctuations affect separately all the quantities introduced above $\left(\mathcal{A}, P_{\text {diff }}, P_{\text {tot }}\right.$, etc. $)$, because of their influence on the dipole scattering amplitudes $\left\langle\mathcal{S}_{N}\right\rangle_{Y_{0}}$ which enter the corresponding formulæ.

Let us conclude this subsection with a warning against the abusive interpretation of the notion of 'inelastic diffraction' in the context of deep inelastic scattering. As we shall see at the beginning of Sect. 4, the diffractive probability (2.3) determines the 'integrated' cross-section for DIS diffraction, i.e., the quantity in the l.h.s. of Eq. (2.1). In view of this, and of the identification in Eq. (2.10), it becomes clear that the separation of $\sigma_{\text {diff }}$ in between an 'elastic' plus an 'integral' piece, as shown in the r.h.s. of Eq. (2.1), corresponds to the decomposition of $P_{\text {diff }}$ in between its elastic and inelastic components, cf. Eq. (2.18): $P_{\text {diff }}=P_{\text {el }}+P_{\text {diff }}^{\text {inel }}$. However, in DIS, and unlike in hadron-hadron collisions, these two pieces of $\sigma_{\text {diff }}$ cannot be separately measured. Indeed, even a process in which the onium scatters elastically (see Fig. 8) still appears as inelastic at the level of the DIS experiment, because the elastically scattered $q \bar{q}$ pair does not recombine back into a virtual photon in the final state, but rather emerges as a hadronic state. Hence, the only experimentally relevant quantity is the 'total' diffractive cross-section, as determined by $P_{\text {diff }}$.

\subsection{Justifying the dipole factorization}

To justify the above formulæ for dipole factorization (in particular, those pertinent to diffraction), we shall use the light-cone wavefunction formalism, as developed in the lightcone gauge $A_{a}^{-}=0$ (which is best suited for a study of the eikonal scattering of the projectile within our conventions). Our presentation will be rather sketchy, as similar techniques and manipulations can be widely found in the literature, and it will focus on the non-trivial aspects of the argument only.

Let us denote by $|\Psi(-\infty)\rangle,\left|\Psi\left(0^{-}\right)\right\rangle,\left|\Psi\left(0^{+}\right)\right\rangle$, and $|\Psi(\infty)\rangle$ the wavefunction of the complete system target plus projectile (including their hadronic descendants in the case of 
the final state) at times ${ }^{8} t=-\infty$ (long before the scattering), $t=0^{-}$(just before the scattering), $t=0^{+}$(immediately after the scattering), and, respectively, $t=\infty$ (the final hadronic state which is measured by the detector). We have:

$$
\left|\Psi\left(0^{-}\right)\right\rangle=\left|\mathcal{O}\left(Y-Y_{0}\right)\right\rangle \otimes\left|h\left(Y_{0}\right)\right\rangle
$$

where the two factors in the r.h.s. are the states of the projectile and, respectively, the target as produced after a rapidity evolution $Y-Y_{0}$ and, respectively, $Y_{0}$.

In the case of the projectile, the initial state for this evolution is the elementary color dipole $(\boldsymbol{x}, \boldsymbol{y})$ produced by the dissociation of the virtual photon: $|\mathcal{O}(0)\rangle=|(\boldsymbol{x}, \boldsymbol{y})\rangle$ (the color indices are kept implicit; see, e.g., Refs. $[46,52]$ for details). The evolved state at $t=0^{-}$is then a superposition of partonic states containing the original $q \bar{q}$ pair $(\boldsymbol{x}, \boldsymbol{y})$ together with an arbitrary number of soft gluons in a given spatial and color configuration. The detailed structure of such individual Fock states turns out not to be necessary for the present purposes. Rather, we shall simply denote by $|N\rangle$ a generic state containing the $q \bar{q}$ pair $(\boldsymbol{x}, \boldsymbol{y})$ together with $N-1$ small- $x$ gluons. We thus write, quite generically:

$$
\left|\mathcal{O}\left(Y-Y_{0}\right)\right\rangle=\sum_{N} c_{N}\left(Y-Y_{0}\right)|N\rangle
$$

where the sum over $N$ should be really understood as a sum over the number of gluons, an integral over their transverse coordinates, and a sum over their polarizations and color configurations (we refer again to Refs. [46,52] for more precise notations).

Concerning the target, its precise initial state at $t=-\infty$ is unimportant here. Rather, all that we need to assume is that at the time of scattering the target can be described as a superposition of states with a given color field (eigenstates of the gauge field operator $\left.A \equiv A^{+}\right)$with coefficients $\Phi[A]$ which depend upon the rapidity $Y_{0}$ :

$$
\left|h\left(Y_{0}\right)\right\rangle=\int D[A] \Phi[A]\left(Y_{0}\right)|A\rangle .
$$

Consider now the onium-hadron collision which takes place at time $t=0$. As anticipated, at high energy the partonic Fock-space states composing the onium are eigenstates of the collision operator: the transverse positions and the spins, or polarizations, of the 'partons' (quarks and gluons) are not changed by the scattering, while their color orientations undergo a field-dependent precession described by Wilson lines. We can write, schematically,

$$
\left|\Psi\left(0^{+}\right)\right\rangle=\int D[A] \Phi[A]\left(Y_{0}\right) \sum_{N} c_{N}\left(Y-Y_{0}\right)\left(\prod_{i=1}^{N} S_{i}[A]\right)|N\rangle \otimes|A\rangle,
$$

where $S_{i}[A]$ is the $S$-matrix for the individual parton $i$, and is a Wilson line (with generally open color indices).

\footnotetext{
8 Strictly speaking, the role of the 'time variables' in the present formalism is played by the light-cone coordinates $-x^{-}$in the case of the projectile and, respectively, $x^{+}$in the case of the target; for simplicity, we shall use the more intuitive notation $t$.
} 
For all the processes that we are interested in, the hadronic target emerges intact from the collision, i.e., it undergoes elastic scattering, so we need only the projection of the outgoing state on the target state prior to the collision:

$$
\begin{aligned}
\left|\Psi_{\text {diff }}\left(0^{+}\right)\right\rangle & \equiv\left|h\left(Y_{0}\right)\right\rangle\left\langle h\left(Y_{0}\right) \mid \Psi\left(0^{+}\right)\right\rangle \\
& =\int D[A]\left|\Phi[A]\left(Y_{0}\right)\right|^{2} \sum_{N} c_{N}\left(Y-Y_{0}\right)\left(\prod_{i=1}^{N} S_{i}[A]\right)|N\rangle \otimes\left|h\left(Y_{0}\right)\right\rangle \\
& \equiv \sum_{N} c_{N}\left(Y-Y_{0}\right)\left\langle\prod_{i=1}^{N} S_{i}[A]\right\rangle_{Y_{0}}|N\rangle \otimes\left|h\left(Y_{0}\right)\right\rangle,
\end{aligned}
$$

where the brackets in the third line refer to the average over the target wavefunction. With the identification $W_{Y_{0}}[A] \equiv\left|\Phi[A]\left(Y_{0}\right)\right|^{2}$, this target averaging is recognized as the color glass averaging in Eq. (2.17).

Eq. (2.25) makes it manifest that, in the calculation of diffractive processes, the average over the target wavefunction is to be performed already at the level of the amplitude [39], rather than at the level of the squared amplitude (which is the quantity defining a probability; see Eq. (2.28) below). This peculiarity is, of course, related to our restriction to processes in which the target undergoes elastic scattering, and would not be true anymore for more general, diffractive, processes in which the target too is allowed to break up after the collision (see also the discussion at the end of this subsection).

Note also that the target averaging in Eq. (2.25) automatically implies a color projection of the outgoing projectile onto color singlet states: Since the post-collisional state of the target (which is the same as its initial state $\left|h\left(Y_{0}\right)\right\rangle$ ) is a color singlet, so must be also the corresponding state of the projectile. In mathematical terms, the weight function $W_{Y_{0}}[A]$ must be gauge-invariant, so the operation of averaging $\prod_{i=1}^{N} S_{i}[A]$ with this weight function must close all the Wilson lines into gauge-invariant color traces. Under this average and for large $N_{c}$, the product $\prod_{i=1}^{N} S_{i}[A]$ naturally reduces to the product of $N$ dipolar factors, where is each of them is like the one appearing in Eq. (2.16).

The final state at the time of detection is obtained by letting the 'diffractive' state in Eq. (2.25) evolve from $t=0$ up to $t=\infty$ under the action of the unitary evolution operator $U(\infty, 0)$ :

$$
\left|\Psi_{\text {diff }}(\infty)\right\rangle=U(\infty, 0)\left|\Psi_{\text {diff }}\left(0^{+}\right)\right\rangle
$$

It is this late time evolution which is responsible for the 'final state interactions' alluded to in the previous subsection: under the action of $U(\infty, 0)$, some of the soft gluons contained in the diffractive state at $t=0^{+}$may recombine with each other, so that the ensuing rapidity gap in a given event (that is, for a given final state) may be actually larger than $Y_{0}$. (See Figs. 6 and 7 for an example.) In fact, the largest allowed gap is equal to $Y$ and corresponds to elastic scattering ${ }^{9}$, i.e., to the situation where all the gluons (or dipoles) in the final state recombine back before their detection into the original dipole $(\boldsymbol{x}, \boldsymbol{y})$. This is depicted in Fig. 8.

9 This is consistent with the fact that, for $Y_{0}=Y$, the diffractive probability (2.3) reduces to the corresponding elastic one, cf. Eq. (2.10) : that is, $P_{\text {diff }}^{\text {inel }}\left(Y=Y_{0}\right)=0$. 


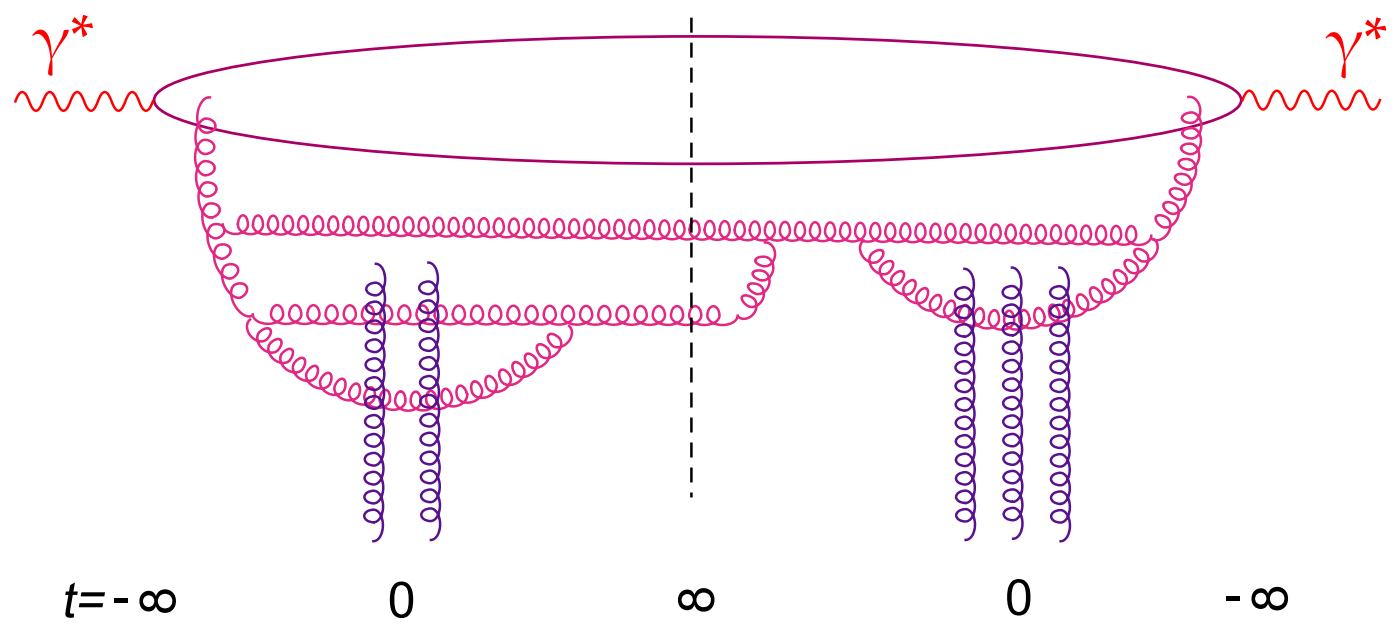

Fig. 6. A particular diagram contributing to the diffractive process $\gamma^{*} h \rightarrow X h$. The dashed vertical line at $t=\infty$ indicates the final (partonic) state. In the direct amplitude, a three-gluon state (together with the original $q \bar{q}$ pair) describes a component of the virtual photon wavefunction which interacts with the target hadron (not shown here) at time $t=0$. After the scattering, final state interactions (gluon recombination) occur and at $t=\infty$ only two gluons are "measured". Similarly, in the complex conjugate amplitude, the wavefunction contains two soft gluons at the time of scattering; after the collision, these gluons recombine with each other, and finally an additional soft gluon is emitted before the time of measurement. The rapidity gap associated with this particular process is larger than $Y_{0}$.

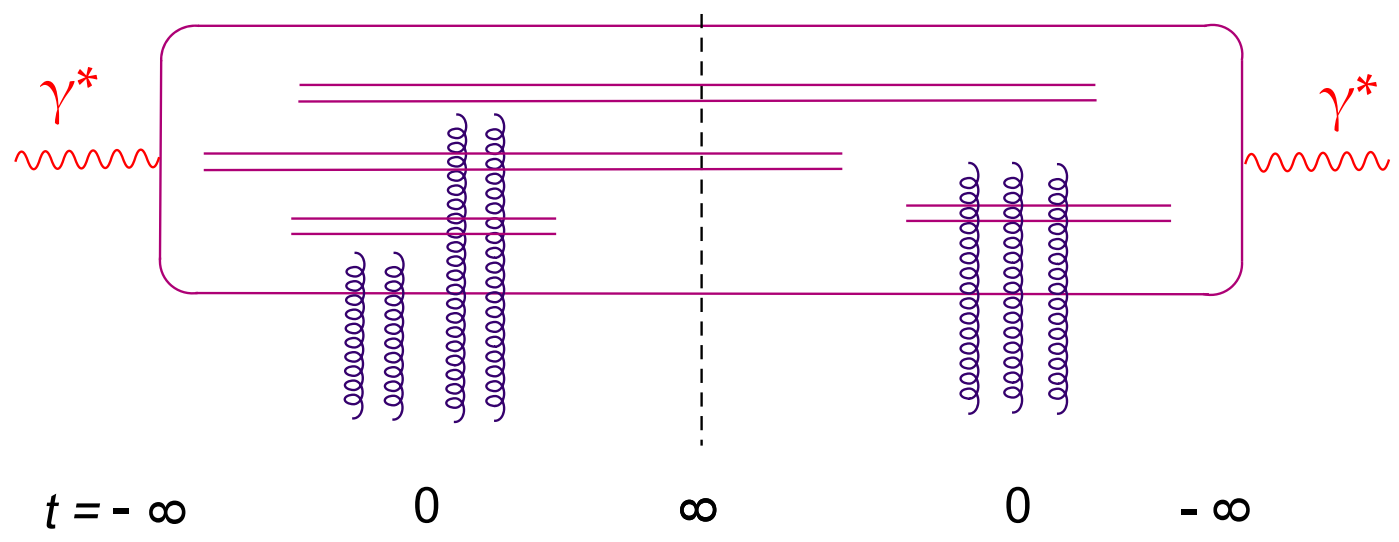

Fig. 7. The same as in Fig. 6 in the large- $N_{c}$ limit; each gluon in the wavefunction of the virtual photon has been replaced with a pointlike quark-antiquark pair in a color octet state.

On the other hand, within the present assumptions, the final gap can clearly not be smaller than $Y_{0}$ : This is so because of our restriction to processes in which the target undergoes elastic scattering, so that none of the virtual quanta initially contained within the target wavefunction (and which are distributed in rapidity from 0 to $Y_{0}$ ) can be released in the final state. Since, on the other hand, there is no activity in the projectile wavefunction at rapidities smaller than $Y_{0}$, it is clear that all the hadrons emerging in the final states and coming from the dissociation of the virtual photon must have a rapidity equal, or superior, to $Y_{0}$. Therefore, the minimal rapidity gap is indeed $Y_{0}$. (See Fig. 9.)

In principle, it should be possible to compute the probability $P_{\text {diff }}\left(\boldsymbol{x}, \boldsymbol{y} ; Y, Y_{\text {gap }}\right)$ for any 


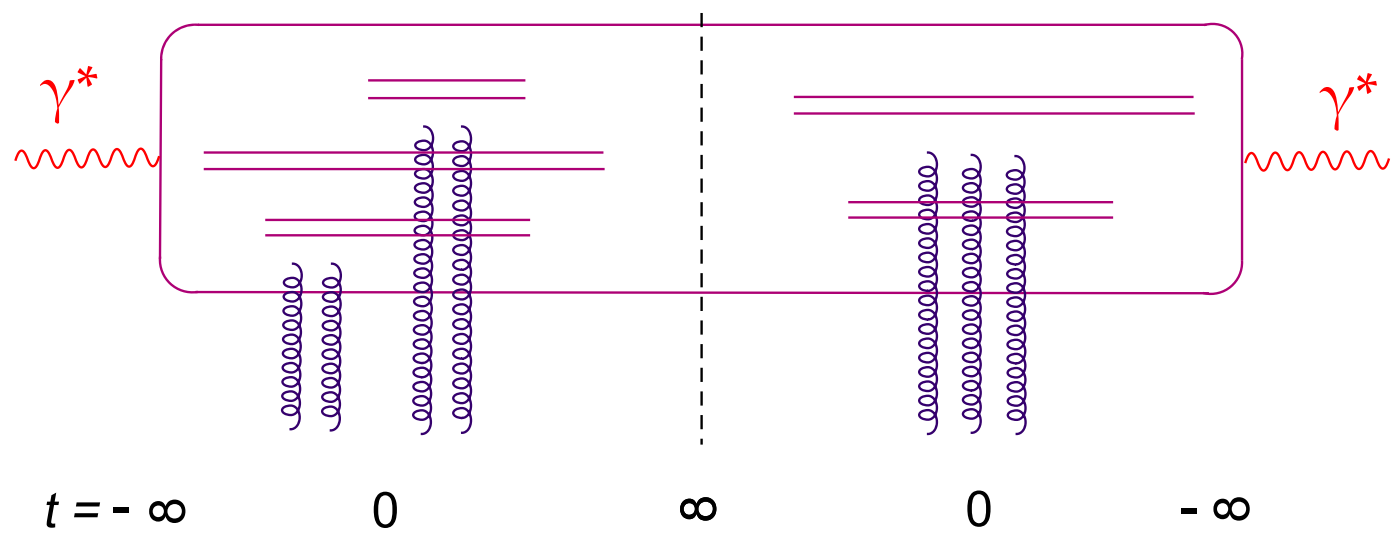

Fig. 8. Another diagram contributing to the diffractive process $\gamma^{*} h \rightarrow X h$. Both the amplitude and the c.c. amplitude correspond to contributions to the forward amplitude (2.9) for dipole-hadron scattering. Hence, this diagram is also an illustration of elastic dipole-hadron scattering, Eq. (2.7). (Notice that this is not an elastic scattering for $\gamma^{*}$ as well, since the virtual photon does not appear in the final state.) Here the rapidity gap takes its maximum value $Y$.

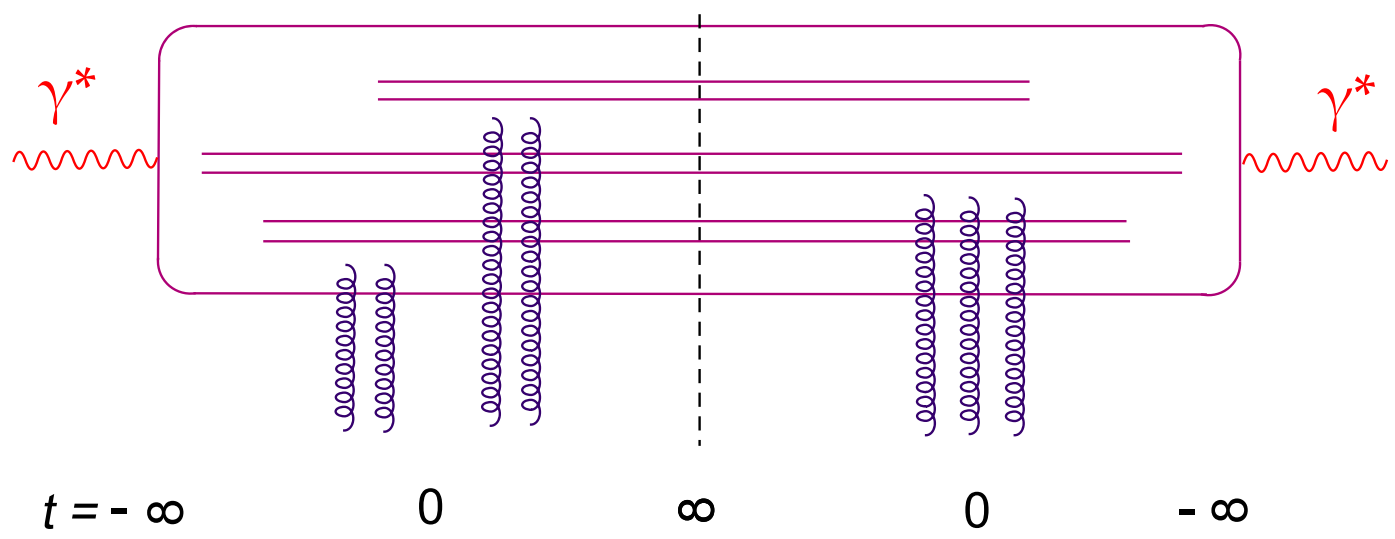

Fig. 9. A final example for the diffractive process $\gamma^{*} h \rightarrow X h$. Here there are no final state interactions at all and the rapidity gap in this case is equal to its minimum value $Y_{0}$.

given value for the minimal rapidity gap $Y_{\text {gap }}$ within the range $Y \geq Y_{\text {gap }} \geq Y_{0}$. To that aim, one should explicitly follow the evolution of the outgoing state after the collision, as indicated in Eq. (2.26), and then project $\left|\Psi_{\text {diff }}(\infty)\right\rangle$ onto final states in which the hadrons released through the fragmentation of the projectile have rapidities $Y \geq Y_{\text {gap }}$. This procedure would select those states within $\left|\Psi_{\text {diff }}\left(0^{+}\right)\right\rangle$in which all the projectile gluons with rapidities between $Y_{0}$ and $Y_{\text {gap }}$ have recombined back in the evolution from $t=0^{+}$up to $t=\infty$. Very recently, a more general formalism has been proposed [76], which in principle allows one to achieve this goal, and thus to compute the diffractive scattering in a generic frame (and for generic values of $N_{c}$ ). It would be interesting to see how our present factorization emerges from that formalism.

However, our main point here is precisely that the calculation of $P_{\text {diff }}\left(\boldsymbol{x}, \boldsymbol{y} ; Y, Y_{\text {gap }}\right)$ can be drastically simplified by conveniently choosing the Lorentz frame - namely, by choosing $Y_{0}=Y_{\text {gap }}$-, since with this particular choice one does not need to worry about the final 
state interactions. Indeed, the condition that the gap be bigger than, or equal to, $Y_{0}$ is automatically satisfied within this kinematics, and it does not introduce any constraint on the final state of the projectile. Hence, the probability for such a diffractive event is measured by the norm $\left\langle\Psi_{\text {diff }}(\infty) \mid \Psi_{\text {diff }}(\infty)\right\rangle$ of the final state, which is the same as the norm of the state $\left|\Psi_{\text {diff }}\left(0^{+}\right)\right\rangle$immediately after the collision, because of the unitarity of the operator $U(\infty, 0)$. One sometimes says that 'the final state interactions cancel between the direct amplitude and the complex conjugate one', but in the present case this cancelation is an almost trivial consequence of our choice for the frame (see, e.g., Refs. [44,77,78] for less trivial cancelations of this type).

More precisely, in computing $P_{\text {diff }}$ one needs to consider the deviation between the outgoing diffractive state and the incoming state $\left|\Psi\left(0^{-}\right)\right\rangle$(indeed, the final state must be different from the initial one in order to have a real scattering). Thus, if one defines:

$$
\left|\delta \Psi_{\text {diff }}\left(0^{+}\right)\right\rangle \equiv\left|\Psi_{\text {diff }}\left(0^{+}\right)\right\rangle-\left|\Psi\left(0^{-}\right)\right\rangle
$$

then in this particular frame one can write

$$
\begin{aligned}
P_{\text {diff }}\left(\boldsymbol{x}, \boldsymbol{y} ; Y, Y_{0}\right) & =\left\langle\delta \Psi_{\mathrm{diff}}\left(0^{+}\right) \mid \delta \Psi_{\mathrm{diff}}\left(0^{+}\right)\right\rangle \\
& =\sum_{N}\left|c_{N}\left(Y-Y_{0}\right)\right|^{2}\left|\left\langle\prod_{i=1}^{N} S_{i}[A]-1\right\rangle_{Y_{0}}\right|^{2},
\end{aligned}
$$

where in writing the second line we have used the fact that the partonic states $|N\rangle$ form an orthonormal basis. At large $N_{c}$, the norm $\left|c_{N}\left(Y-Y_{0}\right)\right|^{2}$ of the state $|N\rangle$ (summed over polarizations and color indices) can be identified with the probability $P_{N}\left(Y-Y_{0}\right)$ for a $N$-dipole configuration in the dipole picture. With this identification, Eq. (2.28) is finally recognized as our originally proposed expression, Eq. (2.3).

The expression (2.9) for the forward scattering amplitude can be similarly obtained : By definition, $\mathcal{A} \equiv 1-\mathcal{S}$ with $\mathcal{S}$ the forward $S$-matrix element, computed as

$$
\begin{aligned}
\mathcal{S}(\boldsymbol{x}, \boldsymbol{y} ; Y) & =\left\langle\Psi\left(0^{-}\right) \mid \Psi\left(0^{+}\right)\right\rangle \\
& =\sum_{N}\left|c_{N}\left(Y-Y_{0}\right)\right|^{2} \int D[A]\left|\Phi[A]\left(Y_{0}\right)\right|^{2} \prod_{i=1}^{N} S_{i}[A],
\end{aligned}
$$

which immediately leads to Eq. (2.9), as anticipated.

The previous discussion also explains our limitation to diffractive processes in which the hadronic target emerges intact from the collision. Of course, it would be very interesting (especially in view of applications to the phenomenology) to be able to describe the more general processes $\gamma^{*} h \rightarrow X Y$ in which the hadron dissociates into some low-mass hadronic system $Y$ separated by a rapidity gap $Y_{\text {gap }}$ from the diffractive state $X$. But to that aim, one cannot avoid a detailed study of the final state interactions, which in the case of the target requires moreover a model for the hadronic structure.

It is finally interesting to compare our formula (2.3) for the diffractive probability to related results in the literature. In the next subsection we shall show that, under an additional, mean field, approximation, our expression for $P_{\text {diff }}$ obeys a non-linear equation originally proposed by Kovchegov and Levin [44]. But before that we would like to point 
out that Eq. (2.3) also encompasses other results from the previous literature. When the target is not too dense so that multiple scattering is negligible, Eq. (2.3) reduces to the expression employed by Bialas and Peschanski [36]. Namely, in the single scattering approximation, we have $\mathcal{A}_{N} \approx \sum_{i=1}^{N} T(i)$ (c.f., Eq. (2.6)), and then Eq. (2.3) becomes

$$
\begin{aligned}
P_{\mathrm{diff}}\left(\boldsymbol{x}, \boldsymbol{y} ; Y, Y_{0}\right) \approx & \int_{\boldsymbol{u}, \boldsymbol{v}} n\left(\boldsymbol{u}, \boldsymbol{v} ; Y-Y_{0}\right)\left|\langle T(\boldsymbol{u}, \boldsymbol{v})\rangle_{Y_{0}}\right|^{2} \\
& +\int_{\boldsymbol{u}_{i}, \boldsymbol{v}_{i}} n^{(2)}\left(\boldsymbol{u}_{1}, \boldsymbol{v}_{1} ; \boldsymbol{u}_{2}, \boldsymbol{v}_{2} ; Y-Y_{0}\right)\left\langle T\left(\boldsymbol{u}_{1}, \boldsymbol{v}_{1}\right)\right\rangle_{Y_{0}}\left\langle T\left(\boldsymbol{u}_{2}, \boldsymbol{v}_{2}\right)\right\rangle_{Y_{0}}^{*},
\end{aligned}
$$

where $n$ and $n^{(2)}$ denote, respectively, the average dipole number density and the average density of (distinct) dipole pairs in the projectile (see, e.g., Sect. 5 Ref. [4] for the corresponding definitions) and the amplitude $\langle T\rangle_{Y_{0}}$ obeys the BFKL equation, as appropriate for a dilute target. Hence, Eq. (2.30) represents the BFKL approximation to $P_{\text {diff }}$. Physically, the first term in the r.h.s. of Eq. (2.30) represents the probability that the same dipole scatters in both the direct and the complex conjugate amplitude, whereas the second term describes the scattering of different dipoles.

On the other hand, for a generic, dense, target but a relatively small rapidity for the projectile, such that $\bar{\alpha}_{s}\left(Y-Y_{0}\right) \ll 1$, one can restrict oneself to onium configurations which contain only two dipoles at the time of scattering. In that case, Eq. (2.3) reduces to results previously obtained by Kovner and Wiedemann [46] and by Munier and Shoshi [50]. The corresponding expressions will be presented in Sect. 4.2, where their high-energy limit will be also investigated.

Let us emphasize here, however, some important differences in our treatment of the target expectation values as compared to Refs. [46,50]. (i) In Ref. [50], the target averages have been estimated in a mean field approximation (cf. Sect. 2.3) which neglects the correlations induced by gluon-number fluctuations; such an approximation is reasonable at intermediate energies, but it eventually fails at sufficiently high energies (see the discussion in Sect. 3). (ii) In an attempt to relax the restriction to elastic scattering on the target side, the authors of Ref. [46] have suggested to replace the target averaging at the level of the amplitude with an averaging at the level of the (diffractive) probability. With our present notations, their suggestion amounts to the following replacement

$$
\left|\langle\mathcal{A}\rangle_{Y_{0}}\right|^{2} \longrightarrow\left\langle\mathcal{A} P_{\operatorname{sing}} \mathcal{A}^{*}\right\rangle_{Y_{0}}
$$

within the r.h.s. of Eq. (2.3). Here, $P_{\text {sing }}$ is the projector onto color singlet states for the projectile wavefunction, and it has been introduced to ensure that $\mathcal{A}$ involves only gaugeinvariant operators; e.g., $P_{\text {sing }} \mathcal{A}=\mathcal{A}_{N}$, cf. Eq. (2.6), for a $N$-dipole state. (In our previous developments, such a projection was automatically ensured by the target averaging at the level of the amplitude, cf. Eq. (2.25).) However, with this new prescription, the target averaging is tantamount to summing over all the possible gauge-invariant final states for the target, without any restriction on their distribution in rapidity. But prior to scattering, the target wavefunction had developed virtual excitations at all the rapidities $y$ ranging from $y=0$ up to $y=Y_{0}$, and in the absence of any rapidity veto on the final state, there is no reason why these quanta should not materialize into hadrons occupying this 
whole rapidity interval. In other terms, with the target expectations values evaluated as in Ref. [46], one is actually including processes which have no rapidity gap whatsoever. Such processes do not qualify as "diffractive" according to the usual terminology, and will be not considered in what follows.

\subsection{Evolution equations in the mean field approximation}

Although our main focus in this paper will be on the effects of fluctuations (i.e., of the deviations from the mean field behaviour) in so far as the target expectation values are concerned, it is nevertheless interesting at this level to slightly deviate from the main stream of the presentation and discuss the mean-field version of our precedent results. This is useful for, at least, two reasons: First, it will allow us to make contact with a non-linear equation for the diffractive probability previously proposed by Kovchegov and Levin [44], and thus clarify the conditions for the validity of the latter. Second, the mean field approximation (MFA) that we shall introduce here will later serve us as a term of comparison, to better emphasize the consequences of the fluctuations at high energy.

Specifically, the MFA consists in the following factorization assumption

$$
\langle S(1) S(2) \cdots S(N)\rangle_{Y_{0}} \approx\langle S(1)\rangle_{Y_{0}}\langle S(2)\rangle_{Y_{0}} \cdots\langle S(N)\rangle_{Y_{0}},
$$

which neglects the correlations among the dipoles induced by their scattering off the target. Ultimately, this is an assumption about the absence of correlations in the target gluon distribution. (For instance, such a factorization holds indeed, at large $N_{c}$, within the framework of the McLerran-Venugopalan model [9] for the gluon distribution of a large nucleus. More generally, it amounts to use a Gaussian approximation for the CGC weight function in Eq. (2.17) [79].) In what follows we shall demonstrate that, with this additional assumption, our formula (2.3) for the diffractive probability obeys indeed to the Kovchegov-Levin (KL) equation of Ref. [44]. During this procedure, and under similar assumptions, we shall also provide a rapid derivation of the Balitsky-Kovchegov (BK) equation for the forward amplitude $[5,10]$.

For more clarity, we shall denote with a bar quantities computed in the mean field approximation. For instance:

$$
\bar{P}_{\text {diff }}\left(\boldsymbol{x}, \boldsymbol{y} ; Y, Y_{0}\right)=\sum_{N=1}^{\infty} \int \mathrm{d} \Gamma_{N} P_{N}\left(\left\{\boldsymbol{z}_{i}\right\} ; Y-Y_{0}\right)\left|1-s_{1} s_{2} \cdots s_{n}\right|^{2}
$$

and similarly

$$
\bar{S}(\boldsymbol{x}, \boldsymbol{y} ; Y)=\sum_{N=1}^{\infty} \int \mathrm{d} \Gamma_{N} P_{N}\left(\left\{\boldsymbol{z}_{i}\right\} ; Y-Y_{0}\right) s_{1} s_{2} \cdots s_{n} .
$$

In these formulæ, $s_{i}$ is a simplified notation for $\left\langle S\left(\boldsymbol{z}_{i-1}, \boldsymbol{z}_{i}\right)\right\rangle_{Y_{0}}$, which in the present context is of course the same as $\bar{S}\left(\boldsymbol{z}_{i-1}, \boldsymbol{z}_{i} ; Y_{0}\right)$.

In what follows, we shall exploit the evolution of the probabilities $P_{N}\left(Y-Y_{0}\right)$ within the dipole picture in order to deduce a set of non-linear evolution equations for the quantities 
defined in Eqs. (2.33)-(2.34). To that aim, it is more convenient to use the original version of the equations for $P_{N}$, due to Mueller [12]. Specifically, Mueller has derived an equation for the following generating functional

$$
Z_{\boldsymbol{x y}}[Y, u]=\sum_{N=1}^{\infty} \int \mathrm{d} \Gamma_{N} P_{N}\left(\boldsymbol{z}_{1} \ldots \boldsymbol{z}_{N-1} ; Y\right) u_{1} u_{2} \cdots u_{N},
$$

where $u_{i} \equiv u\left(\boldsymbol{z}_{i-1}, \boldsymbol{z}_{i}\right)$ is an arbitrary 'source' field, and $(\boldsymbol{x}, \boldsymbol{y})=\left(\boldsymbol{z}_{0}, \boldsymbol{z}_{N}\right)$, as usual. The dipole probabilities $P_{N}(Y)$ can be deduced from $Z_{\boldsymbol{x} \boldsymbol{y}}[Y, u]$ by functionally differentiating with respect to $u$ and then letting $u \rightarrow 0$. The evolution equation satisfied by $Z_{\boldsymbol{x} \boldsymbol{y}}$ reads

$$
\frac{\partial Z_{\boldsymbol{x} \boldsymbol{y}}}{\partial Y}=\frac{\bar{\alpha}_{s}}{2 \pi} \int_{\boldsymbol{z}} \mathcal{M}(\boldsymbol{x}, \boldsymbol{y}, \boldsymbol{z})\left(-Z_{\boldsymbol{x} \boldsymbol{y}}+Z_{\boldsymbol{x} \boldsymbol{z}} Z_{\boldsymbol{z} \boldsymbol{y}}\right),
$$

to be solved with the following initial condition, which follows from Eq. (2.4) :

$$
Z_{\boldsymbol{x y}}[0, u]=u_{\boldsymbol{x} \boldsymbol{y}}
$$

As demonstrated in Ref. [73], this non-linear equation is equivalent with the infinite hierarchy of linear, master, equations for the probability densities $P_{N}$ [16], although the respective pictures of the evolution are quite different: According to Eq. (2.36), the onestep evolution consists in the splitting of the original, high-rapidity, dipole into two child dipoles, which then separately evolve and produce their own distribution of dipoles. By contrast, the master equations of Ref. [16] focus on the splitting of the low-rapidity dipoles produced in the previous steps of the evolution.

Eq. (2.36) looks formally similar to the BK equation for the $S$-matrix [10], and indeed the latter can be easily derived from the former, as we show now: Eqs. (2.34) and (2.35) imply $\bar{S}(\boldsymbol{x}, \boldsymbol{y} ; Y)=Z_{\boldsymbol{x} \boldsymbol{y}}[Y, s]$, which together with Eq. (2.36) immediately leads to (with the simplified notation $\left.\bar{S}_{\boldsymbol{x} \boldsymbol{y}} \equiv \bar{S}(\boldsymbol{x}, \boldsymbol{y} ; Y)\right)$

$$
\frac{\partial \bar{S}_{\boldsymbol{x} \boldsymbol{y}}}{\partial Y}=\frac{\bar{\alpha}_{s}}{2 \pi} \int_{\boldsymbol{z}} \mathcal{M}(\boldsymbol{x}, \boldsymbol{y}, \boldsymbol{z})\left(-\bar{S}_{\boldsymbol{x} \boldsymbol{y}}+\bar{S}_{\boldsymbol{x} \boldsymbol{z}} \bar{S}_{\boldsymbol{z} \boldsymbol{y}}\right)
$$

which is the BK equation $[5,10]$, as anticipated. It is more customary to write this equation in terms of the scattering amplitude $\bar{T}_{\boldsymbol{x} \boldsymbol{y}} \equiv 1-\bar{S}_{\boldsymbol{x} \boldsymbol{y}}$, in which case it reads:

$$
\frac{\partial \bar{T}_{\boldsymbol{x} \boldsymbol{y}}}{\partial Y}=\frac{\bar{\alpha}_{s}}{2 \pi} \int_{\boldsymbol{z}} \mathcal{M}(\boldsymbol{x}, \boldsymbol{y}, \boldsymbol{z})\left(\bar{T}_{\boldsymbol{x} \boldsymbol{z}}+\bar{T}_{\boldsymbol{z} \boldsymbol{y}}-\bar{T}_{\boldsymbol{x} \boldsymbol{y}}-\bar{T}_{\boldsymbol{x} \boldsymbol{z}} \bar{T}_{\boldsymbol{z} \boldsymbol{y}}\right) .
$$

The evolution of the diffractive probability (2.33) can be similarly addressed. Eqs. (2.33) and (2.35) imply (the dependence upon the rapidity gap $Y_{0}$ is kept implicit, since this variable is fixed in the following manipulations)

$$
\begin{aligned}
\bar{P}_{\boldsymbol{x} \boldsymbol{y}}^{\text {diff }}(Y) & =1-2 \bar{S}_{\boldsymbol{x} \boldsymbol{y}}(Y)+Z_{\boldsymbol{x} \boldsymbol{y}}\left[Y, s^{2}\right] \\
& =-1+2 \bar{T}_{\boldsymbol{x} \boldsymbol{y}}(Y)+Z_{\boldsymbol{x} \boldsymbol{y}}\left[Y, s^{2}\right]
\end{aligned}
$$


where we have assumed that $s$ is real, as appropriate at high energy. This rewriting of $\bar{P}_{\boldsymbol{x} \boldsymbol{y}}^{\text {diff }}$ together with the previous equations (2.36) and (2.39) then imply

$$
\begin{aligned}
& \frac{\partial \bar{P}_{\boldsymbol{x y}}^{\text {diff }}}{\partial Y}=2 \frac{\bar{\alpha}_{s}}{2 \pi} \int_{\boldsymbol{z}} \mathcal{M}(\boldsymbol{x}, \boldsymbol{y}, \boldsymbol{z})\left(\bar{T}_{\boldsymbol{x} \boldsymbol{z}}+\bar{T}_{\boldsymbol{z} \boldsymbol{y}}-\bar{T}_{\boldsymbol{x} \boldsymbol{y}}-\bar{T}_{\boldsymbol{x} \boldsymbol{z}} \bar{T}_{\boldsymbol{z} \boldsymbol{y}}\right) \\
& -\frac{\bar{\alpha}_{s}}{2 \pi} \int_{\boldsymbol{z}} \mathcal{M}(\boldsymbol{x}, \boldsymbol{y}, \boldsymbol{z})\left(Z_{\boldsymbol{x} \boldsymbol{y}}\left[s^{2}\right]-Z_{\boldsymbol{x} \boldsymbol{z}}\left[s^{2}\right] Z_{\boldsymbol{z} \boldsymbol{y}}\left[s^{2}\right]\right) \\
& =\frac{\bar{\alpha}_{s}}{2 \pi} \int_{\boldsymbol{z}} \mathcal{M}(\boldsymbol{x}, \boldsymbol{y}, \boldsymbol{z})\left(2 \bar{T}_{\boldsymbol{x} \boldsymbol{z}}+2 \bar{T}_{\boldsymbol{z} \boldsymbol{y}}-2 \bar{T}_{\boldsymbol{x} \boldsymbol{y}}-2 \bar{T}_{\boldsymbol{x} \boldsymbol{z}} \bar{T}_{\boldsymbol{z} \boldsymbol{y}}\right. \\
& \left.-\left(\bar{P}_{\boldsymbol{x} \boldsymbol{y}}^{\text {diff }}+1-2 \bar{T}_{\boldsymbol{x} \boldsymbol{y}}\right)+\left(\bar{P}_{\boldsymbol{x} \boldsymbol{z}}^{\text {diff }}+1-2 \bar{T}_{\boldsymbol{x} \boldsymbol{z}}\right)\left(\bar{P}_{\boldsymbol{z} \boldsymbol{y}}^{\text {diff }}+1-2 \bar{T}_{\boldsymbol{z} \boldsymbol{y}}\right)\right)
\end{aligned}
$$

After some simple manipulations, the expression in the r.h.s. can be recast into the form

$$
\begin{array}{r}
\frac{\partial \bar{P}_{\boldsymbol{x} \boldsymbol{y}}^{\text {diff }}}{\partial Y}=\frac{\bar{\alpha}_{s}}{2 \pi} \int_{\boldsymbol{z}} \mathcal{M}(\boldsymbol{x}, \boldsymbol{y}, \boldsymbol{z})\left(\bar{P}_{\boldsymbol{x} \boldsymbol{z}}^{\mathrm{diff}}+\bar{P}_{\boldsymbol{z} \boldsymbol{y}}^{\mathrm{diff}}-\bar{P}_{\boldsymbol{x} \boldsymbol{y}}^{\mathrm{diff}}+\bar{P}_{\boldsymbol{x} \boldsymbol{z}}^{\mathrm{diff}} \bar{P}_{\boldsymbol{z} \boldsymbol{y}}^{\mathrm{diff}}\right. \\
\left.-2 \bar{T}_{\boldsymbol{x} \boldsymbol{z}} \bar{P}_{\boldsymbol{z} \boldsymbol{y}}^{\mathrm{diff}}-2 \bar{P}_{\boldsymbol{x} \boldsymbol{z}}^{\mathrm{diff}} \bar{T}_{\boldsymbol{z} \boldsymbol{y}}+2 \bar{T}_{\boldsymbol{x} \boldsymbol{z}} \bar{T}_{\boldsymbol{z} \boldsymbol{y}}\right)
\end{array}
$$

which is recognized, as anticipated, as the equation proposed by Kovchegov and Levin [44]. It is interesting to notice that, in Ref. [44], this equation has been obtained by working in the target rest frame, which required a more intricate analysis of the final state interactions.

The non-linear terms in Eqs. (2.39) and (2.42) describe (incoherent) multiple scattering between the dipoles in the projectile and the color fields in the target and are responsible for unitarization: In the high energy limit, the solutions $\bar{T}(Y)$ and $\bar{P}_{\text {diff }}\left(Y, Y_{0}\right)$ approach the 'black-disk' fixed points $\bar{T}=\bar{P}_{\text {diff }}=1$, in agreement with the corresponding properties of the more general formulæ (2.3) and (2.9).

But although they do respect the unitarity bounds, Eqs. (2.39) and (2.42) cannot be used in a study of the high-energy limit, because of the mean field approximation (2.32) inherent in their derivation and which fails at high energy $[1,2]$. To properly include the relevant fluctuations, one must replace the BK equation with the hierarchy of Pomeron loop equations $[4,14]$ for the $N$-dipole amplitudes $\langle S(1) S(2) \cdots S(N)\rangle_{Y}$. Once these amplitudes are thus computed, they can be used to evaluate the diffractive probability according to Eq. (2.3), which replaces the solution to the KL equation for sufficiently high energy. Although the general solution to the Pomeron loop equations is not known, the correspondence between high-energy QCD and problems in statistical physics [3,33] has allowed one to deduce valuable information about the behaviour of the dipole amplitudes in the high-energy limit [2-4]. This will be explained in the next section. 


\section{Dipole amplitudes at high energy: Fluctuations \& Diffusive scaling}

The essential ingredient required by a calculation of the various scattering probabilities introduced in the previous section are the $N$-dipole amplitudes $\left\langle T^{(N)}\right\rangle_{Y}$ which describe the scattering between the target and a system of dipoles. In this section, we shall describe the calculation of these amplitudes in the high-energy regime where the MFA breaks down, because of the strong influence of gluon-number fluctuations [2-4].

Most of the results to be presented below have already appeared in Refs. [2-4], but since these are recent developments and, moreover, are of utmost importance for the present analysis, our respective discussion here will be quite complete. In doing so, we shall also clarify some points which have not been addressed in the previous studies, like the borderline between the intermediate-energy regime, where the BK equation is a reasonable approximation and geometric scaling applies, and the high-energy regime, where the evolution is dominated by fluctuations leading to diffusive scaling.

The subsequent picture will be given in a frame in which all of the energy is carried by the hadronic target, so the projectile is a bare dipole, or a set of few such dipoles. To compute a target-averaged amplitude like $\left\langle T^{(N)}\right\rangle_{Y}$, one needs to (i) evaluate the scattering amplitude $T_{Y}(\boldsymbol{x}, \boldsymbol{y})$ for a single dipole and in a single event (meaning, for a given configuration of the gluon fields in the target, as generated through a single evolution from $y=0$ up to $y=Y$ ) and (ii) average the result over the ensemble of events (that is, over all the possible target evolutions from $y=0$ to $y=Y$ ). It turns out that, for sufficiently large values of $Y$, both these operations become relatively simple and their results are universal (i.e., independent of the initial conditions at low energy), as they are fully determined by the quantum evolution with $Y[2-4]$. In fact, this universality is even stronger, in the sense that the details of the evolution matter for a couple of parameters which enter the final results (namely, the coefficients $\lambda$ and $D_{\text {fr }}$ which appeared in the Introduction), but not for the functional form of the average amplitudes [4].

\subsection{The event-by-event dipole amplitude}

From now on, we shall neglect any non-trivial dynamics in the impact parameter space, that is, we shall assume that the evolution with increasing energy is quasi-local in $b$ and we shall often omit the $b$-argument from the amplitudes. This is a reasonable approximation so long as one is interested (as we are here) in the high-energy limit of the DIS crosssections at fixed impact parameter. Then, the dipole amplitude in a single event can be written as

$$
T_{Y}(\boldsymbol{x}, \boldsymbol{y}) \equiv T_{Y}(r) \equiv T_{Y}(\rho)
$$

where $r=|\boldsymbol{x}-\boldsymbol{y}|$ is the dipole size and $\rho$ represents $r$ in logarithmic units, like in Fig. $1: \rho \equiv \ln \left(1 / r^{2} Q_{0}^{2}\right)$, with $Q_{0}$ a scale of reference introduced by the initial conditions at low energy. Note that large $\rho$ corresponds to small dipole sizes, or to large transverse momenta $\left(\rho \sim \ln k_{\perp}^{2}\right)$ after a Fourier transform. 
For sufficiently high energy, the amplitude takes the form of a traveling wave [33] :

$$
T_{Y}(\rho) \simeq T\left(\rho-\rho_{s}(Y)\right)
$$

This is a front which interpolates between a strong scattering regime at $\rho<\rho_{s}(Y)$, where the unitarity bound $T=1$ has been saturated, and a weak scattering regime at $\rho>\rho_{s}(Y)$, where $T_{Y} \ll 1$, and which propagates towards larger values of $\rho$ when increasing $Y$. Here, $\rho_{s}(Y)$ is the position of the front - conventionally defined as the dipole size $\rho$ for which $T(\rho)=1 / 2$ - and it increases linearly with $Y: \rho_{s}(Y) \simeq \lambda \bar{\alpha}_{s} Y$. The position of the front also serves as a definition for the target saturation momentum:

$$
\rho_{s}(Y) \equiv \ln \left(Q_{s}^{2}(Y) / Q_{0}^{2}\right) \Longrightarrow Q_{s}^{2}(Y) \simeq Q_{0}^{2} \mathrm{e}^{\lambda \bar{\alpha}_{s} Y}
$$

Accordingly, $\lambda$ will be alternatively referred to as the saturation exponent, or the front velocity. The BK equation (2.39) predicts $\lambda=\lambda_{0} \approx 4.88$ [61,62], but this result is significantly lowered by gluon number fluctuations $[2,3]$ (see below) and also by the next-toleading order corrections in perturbative QCD [63].

We shall also need later some analytic control over the shape of an individual front. As indicated in Eq. (3.2), this depends only upon the difference $\rho-\rho_{s}(Y)$, meaning that the front propagates without distortion: in a co-moving frame, the shape of the front is independent of $Y$. This property has been first noticed within the framework of the mean field approximation, that is, for the solution to the BK equation [33,61,62, 80-84], but for the individual fronts this remains true also in the presence of fluctuations $[3,66]$. Since $\rho-\rho_{s}=\ln \left(1 / r^{2} Q_{s}^{2}\right)$, we deduce that the dipole amplitude $T_{Y}(r)$ depends upon its two kinematical variables $r$ and $Y$ only trough the dimensionless product $r^{2} Q_{s}^{2}(Y)-$ the property usually referred to as geometric scaling [33,60-63]. This property has far reaching physical consequences, as it provides a natural explanation [61] for a new scaling behaviour observed in the small- $x$ data at HERA [60] and plays also an essential role in understanding the particle production in deuteron-gold collisions at RHIC [85, 86]. Note, however, that the quantity which matters for the calculation of physical observables is the target-averaged dipole amplitude $\langle T(\rho)\rangle_{Y}$ and, as we shall later explain, the geometric scaling property characteristic of a single event does not necessarily translate to the average quantities, because of fluctuations [2-4].

The fluctuations are inherent in the evolution with increasing energy, because the instantaneous configuration of the gluons in the target (as probed by the external dipole) can abruptly change from one rapidity step to another via the emission of new gluons. At high energy, the fluctuations are relatively important only in the dilute regime at large transverse momenta $\left(k_{\perp} \gg Q_{s}\right)$, where the gluon occupation numbers are small. Since the high $-k_{\perp}$ gluons scatter preponderantly with small dipoles, of size $r \sim 1 / k_{\perp}$, we deduce that the fluctuations should significantly affect only the tail of the dipole amplitude $T_{Y}(\rho)$ at large values of $\rho$. Simple physical considerations $[3,66]$ (see also below) show that the fluctuations have a relatively small effect on the overall shape of an individual front they modify only the foremost part of the front, where $T$ is very small: $T \sim \alpha_{s}^{2}-$, but they strongly influence the dynamics of the front, thus considerably reducing its velocity $\lambda$ with respect to the mean field prediction $\lambda_{0}$. This behaviour, which is confirmed by numerical simulations within both QCD and statistical physics (see, e.g., [28, 29, 66, 87]), 
reflects the pulled nature of the saturation front: the propagation is entirely driven by the dynamics in the tail of the front at $\rho \gg \rho_{s}(Y)$, and thus is very sensitive to even small changes in this tail due to fluctuations.

Returning to a discussion of the shape of the front, it is important to stress here (in anticipation of Sect. 3.2) that, in order to compute the average amplitudes at high energy, one needs only a very limited information about the shape of the individual fronts: it is in fact enough to know that $T_{Y}(\rho)=1$ for $\rho<\rho_{s}(Y)$ [4]. But precisely in order to develop the corresponding argument in Sect. 3.2, and also in view of numerical tests in the forthcoming sections, we shall need to know a little bit more about the function $T_{Y}(\rho)$. Specifically, the following piecewise approximation, which can be inferred from the relevant literature $[3,66,67]$, will be sufficient for our purposes:

$$
T(z)= \begin{cases}1 & \text { for } z<0 \\ A \mathrm{e}^{-\gamma_{0} z} & \text { for } 1<z<L \\ B \mathrm{e}^{-z} & \text { for } L \ll z .\end{cases}
$$

Here, $z \equiv \rho-\rho_{s}$ is the scaling variable, $A$ and $B$ are undetermined normalization factors, $\gamma_{0} \approx 0.63$, and $L$ is the 'width of the front', namely, the distance $\rho-\rho_{s}$ over which the amplitude falls off from its saturation value $T=1$ to a value of order $\alpha_{s}^{2}$, where the fluctuations become important. This condition immediately yields:

$$
L \simeq \frac{1}{\gamma_{0}} \ln \frac{1}{\alpha_{s}^{2}}+O(1)
$$

Note that the physical regions in which the solution is approximately given have no overlap with each other, so, not surprisingly, the expressions shown in Eq. (3.4) do not provide a continuous interpolation for $T(z)$. The first line in Eq. (3.4) is, clearly, the saturation region; the second line describes the front of the traveling wave, with the characteristic 'anomalous dimension' $1-\gamma_{0} \approx 0.37$ which is a hallmark of the BFKL evolution in the presence of saturation [61,62]; finally, the third line represents the perturbative QCD tail, where the amplitude exhibits color transparency $\left(T(r) \sim r^{2}\right)$, and which is generated via the large $-\rho$ behaviour of the BFKL kernel. More precisely, within the BFKL evolution, the color transparency is amended by quantum corrections in the "double-logarithmic approximation" (DLA). These are not explicitly shown here, since the contribution of this large $-\rho$ tail to physical quantities is anyway negligible in the high-energy regime of interest. It is however important to notice that such DLA effects violate geometric scaling, which is therefore restricted to the region at $z \lesssim L$.

The single-event amplitude in Eq. (3.4) looks quite similar to the solution to the BK equation (see, e.g., Ref. [86]), but it differs from the latter in two essential points:

a) The front is compact in Eq. (3.4), i.e., it has a finite width $L$ which is independent of $Y$. By contrast, the front of the BK solution extends up to distances $\rho-\rho_{s} \sim \sqrt{Y}$, and thus its width increases with $Y$. In the context of the BK equation, this front is often referred to as the geometric scaling window [61,62]. We see that, as a consequence of fluctuations, the width of the geometric scaling window for a single event (and for sufficiently high 
energy) is considerably reduced with respect to the mean field approximation.

b) The front velocity $\lambda$ which enters Eq. (3.4) via $\rho_{s}$ is considerably smaller than the corresponding BK velocity $\lambda_{0}$. This is seen numerically for generic values of $\alpha_{s}$, and is confirmed by an analytic calculation valid in the weak coupling limit $\alpha_{s}^{2} \rightarrow 0$, which shows that in this limit $\lambda$ converges towards $\lambda_{0}$, but only very slowly $[2,3,66]$ :

$$
\lambda \simeq \lambda_{0}-\frac{\mathcal{C}}{\ln ^{2}\left(1 / \alpha_{s}^{2}\right)} \quad \text { when } \quad \alpha_{s}^{2} \ll 1 .
$$

Here, $\mathcal{C}$ is a known number, which is determined by the linear, BFKL, dynamics and turns out to be quite huge : $\mathcal{C}=\pi^{2} \gamma_{0} \chi^{\prime \prime}\left(\gamma_{0}\right) \approx 150$. (Here, $\chi(\gamma)$ is the Mellin transform of the BFKL kernel $[2,3]$. Note also that $\lambda_{0}=\chi\left(\gamma_{0}\right) / \gamma_{0} \approx 4$.88.) The tendency of $\lambda$ to decrease when increasing $\alpha_{s}^{2}$ is also confirmed by a study of the 'strong noise limit', which shows that in that limit $\lambda$ vanishes as a power of $1 / \alpha_{s}^{2}[88]$.

More precisely, the above picture applies only for high enough rapidities, larger than the formation time of the front, estimated as $[3,89]$ :

$$
\bar{\alpha}_{s} Y_{\text {form }} \sim \frac{\ln ^{2}\left(1 / \alpha_{s}^{2}\right)}{2 \chi^{\prime \prime}\left(\gamma_{0}\right) \gamma_{0}^{2}} .
$$

This is the rapidity evolution necessary for the front to reach its final form in Eq. (3.4), and for the velocity to reach its asymptotic value in Eq. (3.6), starting with some generic initial condition at $Y=0$. For $Y<Y_{\text {form }}$, the width of the front increases with $Y$ as $\rho-\rho_{s} \sim \sqrt{Y}$, via BFKL diffusion, while for $Y>Y_{\text {form }}$, this width gets stuck at its maximal value, equal to $L$. As indicated by the 'geometric scaling' region in the diagram in Fig. 1, a similar behaviour is shown also by the average amplitude $\langle T(\rho)\rangle_{Y}$, but only up to some maximal rapidity $Y \sim Y_{\mathrm{DS}}$, beyond which the effects of fluctuations become overwhelming (see the discussion in the next subsection).

Let us conclude this presentation with a few physical considerations on the role of fluctuations [3]. The previous arguments exhibit the special role played by the parameter $\alpha_{s}^{2}$ in the study of fluctuations (in particular, the mean field limit is obtained as $\alpha_{s}^{2} \rightarrow 0$ ), and this deserves a comment. In the dilute regime where the fluctuations are important, and for large $N_{c}$, the target itself can be described as a collection of dipoles, and then the amplitude $T(r)$ describes the scattering between the external dipole $r$ and the target dipoles. The dipole-dipole amplitude $T\left(r, r^{\prime}\right)$ is of order $\alpha_{s}^{2}$ and is peaked at sizes $r^{\prime} \sim r$. Hence, to a good approximation, $T(r) \sim \alpha_{s}^{2} f(r)$, where $f(r)$ is the dipole occupation number in the target, that is, the number of dipoles with size $r$ (per unit of $\ln 1 / r^{2}$ ) within an area $r^{2}$ around the impact parameter of the external dipole. In a given event, $f$ is discrete $(f=0,1,2, \ldots)$, so the scattering amplitude takes on only discrete values, which are multiples of $\alpha_{s}^{2}$. In particular, the minimal non-zero value for $T$ is $\alpha_{s}^{2}$, showing that the tail of the front must abruptly end when $T$ becomes of $O\left(\alpha_{s}^{2}\right)$. This explains the compact nature of the front.

Furthermore, the particle number fluctuations follow a normal distribution: $\delta f \sim \sqrt{f}$, showing that $\delta T \sim \alpha_{s}^{2} \sqrt{f} \sim \sqrt{\alpha_{s}^{2} T}$. Thus, the mean field approximation becomes reliable, in the sense that $\delta T \ll T$, as soon as $T \gg \alpha_{s}^{2}$. In particular, this becomes a good 
approximation everywhere (i.e., for any $r$ ) in the limit $\alpha_{s}^{2} \rightarrow 0$.

\subsection{Front dispersion through fluctuations}

Let us consider now the average over the target wavefunction, which in the present context amounts to an average over the statistical ensemble of fronts : the fronts associated to all the possible evolutions of the target over a rapidity interval $Y$. It turns out that at high energy — sufficiently high for the fronts to reach their canonical form, cf. Eq. (3.7) - this averaging is quite simple: Since all the fronts in the ensemble have the same shape, they can differ from each other only by a translation. That is, the only random variable in the problem is the position $\rho_{s}$ of the front, which can be argued to be distributed according to the following, Gaussian, probability density [3, 66, 67]

$$
P_{Y}\left(\rho_{s}\right)=\frac{1}{\sqrt{\pi} \sigma} \exp \left[-\frac{\left(\rho_{s}-\left\langle\rho_{s}\right\rangle\right)^{2}}{\sigma^{2}}\right]
$$

In this equation, $\left\langle\rho_{s}\right\rangle \simeq \lambda \bar{\alpha}_{s} Y$ is the average position of the front and increases with $Y$ with the velocity shown in Eq. (3.6), since this is the common average velocity for all the fronts in the ensemble. Furthermore, $\sigma^{2} / 2 \equiv\left\langle\rho_{s}^{2}\right\rangle-\left\langle\rho_{s}\right\rangle^{2}$ is the front dispersion, which rises linearly with $Y, \sigma^{2}(Y) \simeq D_{\text {fr }} \bar{\alpha}_{s} Y$, since the stochastic process is a random walk around the average front ${ }^{10}$. It has been first suggested by numerical simulations [66], and very recently confirmed through analytic arguments [67] that, in the weak coupling limit $\alpha_{s}^{2} \rightarrow 0$, the front diffusion coefficient $D_{\text {fr }}$ scales as

$$
D_{\mathrm{fr}} \simeq \frac{\mathcal{D}}{\ln ^{3}\left(1 / \alpha_{s}^{2}\right)} \quad \text { when } \quad \alpha_{s}^{2} \ll 1
$$

This vanishes, as expected, in the mean field limit $\alpha_{s}^{2} \rightarrow 0$, but only very slowly. The coefficient $\mathcal{D}$ has been explicitly computed in Ref. [67]. (Once again, this is determined by the linear dynamics in the tail.)

Then the average amplitude $\langle T\rangle_{Y}$ is determined by

$$
\langle T(\rho)\rangle_{Y}=\int_{-\infty}^{\infty} \mathrm{d} \rho_{s} P_{Y}\left(\rho_{s}\right) T\left(\rho-\rho_{s}\right)
$$

with $T\left(\rho-\rho_{s}\right)$ the single-event front in Eq. (3.4). Higher-point correlations can be computed similarly. Note that the ensuing average amplitudes will naturally depend upon the difference $z \equiv \rho-\left\langle\rho_{s}\right\rangle$, but they will also show additional dependencies upon $Y$, via the front dispersion $\sigma$. That is, unlike the individual fronts, the average amplitudes will generally not show geometric scaling $[2,3]$. In fact, as we shall shortly discover, at sufficiently high energies the geometric scaling is replaced by a new, diffusive, scaling.

\footnotetext{
${ }^{10}$ Note that our present normalizations for the coefficients $\lambda$ and $D_{\text {fr }}$ differ by a factor $\bar{\alpha}_{s}$ from those used in the Introduction.
} 
Given the Gaussian nature of the probability distribution (3.8), it is straightforward to compute the average in Eq. (3.10) for the piecewise-defined front in Eq. (3.4), and we shall do so indeed in the more detailed studies in the next subsections. Here, however, we would like to point out (following an original analysis in Ref. [4]) that, for sufficiently high energy, the dominant contributions to the average amplitudes $\left\langle T^{(N)}\right\rangle_{Y}$ with any $N$ are given by the saturation region in Eq. (3.4), and thus they are all expressed through a universal function.

Let us start by better specifying what we mean by "sufficiently high energy" : As anticipated in the Introduction (cf. Fig. 1), this is the regime in which the target expectation values are dominated by dense fluctuations, which in turn requires relatively high values of $Y$ and not too large values of $\rho$ (but $\rho$ can still be much larger than the average saturation scale $\left\langle\rho_{s}\right\rangle$; see below). More precisely, we shall demonstrate here that within the high-energy regime defined as

$$
\text { High-energy regime } \Longleftrightarrow \sigma \gg 1 / \gamma_{0} \text { and } \rho-\left\langle\rho_{s}\right\rangle \ll \gamma_{0} \sigma^{2} \text {, }
$$

the dominant contributions to the average dipole amplitudes can be simply computed with a $\Theta$-function saturation front:

$$
T_{\theta}(\rho) \equiv \Theta\left(\rho_{s}-\rho\right)
$$

which in turn implies [4]

$$
\langle T(\rho)\rangle_{Y}=\frac{1}{2} \operatorname{Erfc}\left(\frac{z}{\sigma}\right)
$$

and, more generally,

$$
\left\langle T\left(\rho_{1}\right) T\left(\rho_{2}\right) \cdots T\left(\rho_{N}\right)\right\rangle_{Y}=\frac{1}{2} \operatorname{Erfc}\left(\frac{z_{>}}{\sigma}\right)
$$

where $z=\rho-\left\langle\rho_{s}\right\rangle$ and $z_{>}=\rho_{>}-\left\langle\rho_{s}\right\rangle$, with $\rho_{>}=$the largest among the $\rho_{i}$ 's. In these equations, $\operatorname{Erfc}(\mathrm{x})$ is the complementary error function,

$$
\operatorname{Erfc}(x) \equiv \frac{2}{\sqrt{\pi}} \int_{x}^{\infty} \mathrm{d} t \mathrm{e}^{-t^{2}}
$$

which arises here as the integral of the probability density (3.8) over all values of $\rho_{s}$ larger than $\rho$ (respectively, $\rho_{>}$): The r.h.s. of Eq. (3.13) is simply the probability to find a front which is at saturation on the resolution scale $\rho$.

Eqs. (3.13)-(3.14) show that the average scattering amplitudes at high energy are dominated by those fronts within the statistical ensemble at $Y$ which are at saturation at the highest resolution scale $\rho_{>}$set by the incoming projectile. This is true, in particular, for relatively large values of $\rho$, well above $\left\langle\rho_{s}\right\rangle$, where on the average the scattering is weak, $\langle T(\rho)\rangle_{Y} \ll 1$, yet this average value is dominated by the relatively rare configurations ('black spots') which are at saturation for that (large) value of $\rho$. The subdominant con- 
tributions not shown in Eqs. (3.13)-(3.14) are suppressed by, at least, one power of ${ }^{11} 1 / \sigma$ and/or $z / \sigma^{2}$ (respectively, $z_{>} / \sigma^{2}$ ), and are sensitive to the detailed shape of the singleevent front. A similar sensitivity holds for the average amplitudes outside the high-energy regime defined in Eq. (3.11).

Note that the universal behaviour exhibited in Eqs. (3.13)-(3.14) emerges also in the limit of strong fluctuations, as recently shown in Ref. [88]. This observation is consistent with the analysis in Ref. [4] and the present considerations, since the limit of strong fluctuations is formally obtained by letting $\sigma \rightarrow \infty$ within our formulæ, in which case the 'high-energy regime' of Eq. (3.11) extends everywhere.

To demonstrate the above results, we need to study the influence of the shape of the front on the average amplitudes with increasing energy. To that aim, it is sufficient to consider the following, simplified, profile for an individual event :

$$
T_{\gamma}(\rho)=\Theta\left(\rho_{s}-\rho\right)+\Theta\left(\rho-\rho_{s}\right) \mathrm{e}^{-\gamma\left(\rho-\rho_{s}\right)} .
$$

For $\gamma=\gamma_{0} \approx 0.63$, this simulates the front region in the second line of Eq. (3.4), while for $\gamma=1$ it rather corresponds to the 'color transparency' region in the third line there. By using Eqs. (3.16) and (3.8) it is straightforward to deduce

$$
\langle T(z)\rangle_{Y}=\frac{1}{2} \operatorname{Erfc}\left(\frac{z}{\sigma}\right)+\frac{1}{2} \exp \left(\frac{\gamma^{2} \sigma^{2}}{4}-\gamma z\right)\left[2-\operatorname{Erfc}\left(\frac{z}{\sigma}-\frac{\gamma \sigma}{2}\right)\right],
$$

with $z=\rho-\left\langle\rho_{s}\right\rangle$. The first term is the same as in Eq. (3.13) and comes from the saturation piece of $T_{\gamma}$, while the second term comes from the exponential piece of Eq. (3.16).

The following limiting behaviours of the function $\operatorname{Erfc}(x)$ will be useful in what follows:

$$
\operatorname{Erfc}(x)= \begin{cases}2-\frac{\exp \left(-x^{2}\right)}{\sqrt{\pi}|x|} & \text { for } x \ll-1 \\ 1 & \text { for } x=0 \\ \frac{\exp \left(-x^{2}\right)}{\sqrt{\pi} x} & \text { for } x \gg 1\end{cases}
$$

i) Consider first the (relatively) low energy situation at $\sigma \ll 1 / \gamma$. By using Eqs. (3.17)(3.18) one finds that the average amplitude retains its single event profile, except in the short interval $|z| \lesssim \sigma$ where it gets smoothed:

$$
\langle T(z)\rangle_{Y}=\left\{\begin{array}{ll}
1 & \text { for } z \ll-\sigma, \\
\mathrm{e}^{-\gamma z} & \text { for } \sigma \ll z,
\end{array} \quad \text { for } \quad \sigma \ll 1 / \gamma .\right.
$$

In this regime, geometric scaling is manifest at the level of the average amplitudes. The situation is similar to the mean field approximation, except for the facts that the real front is compact and has a smaller velocity, cf. Eq. (3.6). (The compactness becomes manifest,

${ }^{11}$ Such estimates should more properly read, e.g., $z / \gamma_{0} \sigma^{2}$, but here and in what follows we shall often use the fact that $\gamma_{0} \approx 0.63$ is a number of $O(1)$ to simplify the parametric estimates. 
of course, only if one starts with the more realistic profile in Eq. (3.4).) We thus conclude

$$
\text { Negligible dispersion } \Longleftrightarrow \sigma \ll 1 / \gamma_{0} \quad \Longleftrightarrow \quad \bar{\alpha}_{s} Y \ll \frac{\ln ^{3}\left(1 / \alpha_{s}^{2}\right)}{\gamma_{0}^{2} \mathcal{D}} .
$$

The upper limit above, namely $\bar{\alpha}_{s} Y_{\mathrm{DS}} \sim \ln ^{3}\left(1 / \alpha_{s}^{2}\right)$, is parametrically larger than the 'critical' rapidity $\bar{\alpha}_{s} Y_{c} \sim \ln \left(1 / \alpha_{s}^{2}\right)$ for the onset of the unitarity corrections, cf. Eq. (2.13), and also than the formation time $\bar{\alpha}_{s} Y_{\text {form }} \sim \ln ^{2}\left(1 / \alpha_{s}^{2}\right)$, cf. Eq. (3.7), for the individual fronts (as it should for the present discussion to make sense).

ii) For $\sigma \gg 1 / \gamma$, the first term in Eq. (3.17) dominates everywhere except at extremely large distances ahead of the front, such that $z \gtrsim \gamma \sigma^{2}$. For instance, in the interesting range $\sigma \ll z \ll \gamma \sigma^{2}$, where the average amplitude is small, we obtain

$$
\langle T(z)\rangle_{Y} \simeq \frac{1}{2 \sqrt{\pi}} \frac{\sigma}{z}\left\{1+\frac{2 z}{\gamma \sigma^{2}}\right\} \mathrm{e}^{-z^{2} / \sigma^{2}} \quad \text { for } \quad \sigma \ll z \ll \gamma \sigma^{2},
$$

where the first term inside the curly brackets comes from the 'saturation' piece in the r.h.s. of Eq. (3.17), while the second term corresponds to the 'exponential' piece there, and is suppressed with respect to the first term by a factor $z / \gamma \sigma^{2} \ll 1$, as anticipated. One can similarly check that Eq. (3.14) yields indeed the dominant behaviour for the $N$-dipole amplitude $\left\langle T^{(N)}\right\rangle_{Y}$ within the range specified by Eq. (3.11).

On the other hand, it is easy to check that, for much larger values of $\rho$, such that $z \gg$ $\sigma^{2}$, the dominant contribution to the average amplitude comes from the second term in Eq. (3.17), that is, from the exponential tail in Eq. (3.16). One then finds

$$
\langle T(z)\rangle_{Y} \simeq \exp (-\gamma z) \exp \left(\frac{\gamma^{2} \sigma^{2}}{4}\right) \quad \text { for } \quad z \gg \sigma^{2}
$$

where we recognize the same exponential decay with $\rho$ as for a single front, Eq. (3.16).

The piecewise expression of the average amplitude at high energy reads therefore

$$
\langle T(z)\rangle_{Y}=\left\{\begin{array}{ll}
(1 / 2) \operatorname{Erfc}(z / \sigma) & \text { for }-\infty<z \ll \gamma_{0} \sigma^{2}, \\
\sim \mathrm{e}^{-z} & \text { for } \gamma_{0} \sigma^{2} \ll z,
\end{array} \quad \text { for } \sigma \gg 1 / \gamma_{0},\right.
$$

where we have directly considered the more realistic profile in Eq. (3.4), so the exponential tail at very large $z$ is determined by the 'color transparency' piece of Eq. (3.4).

For all but very large values of $z$, the average front profile in Eq. (3.23) has no resemblance at all with the single-event profile in Eq. (3.4): There is no trace of the BFKL 'anomalous dimension' $\gamma_{0}$, nor of 'geometric scaling'. Rather, so long as $z \ll \gamma_{0} \sigma^{2}$, the average amplitude obeys a new type of scaling - it scales as a function of $z / \sigma$ - which was previously observed in Refs. [3,4], and for which we propose the name of diffusive scaling. Note that the kinematical region for diffusive scaling coincides with the high-energy regime of Eq. (3.11), and is represented as the region at $Y>Y_{\mathrm{DS}}$ and $\rho$ at the left of the curve " $Q_{d}^{2}$ " in Fig. 1. In particular, within the weak scattering regime at $\sigma \ll z \ll \gamma_{0} \sigma^{2}$, the 


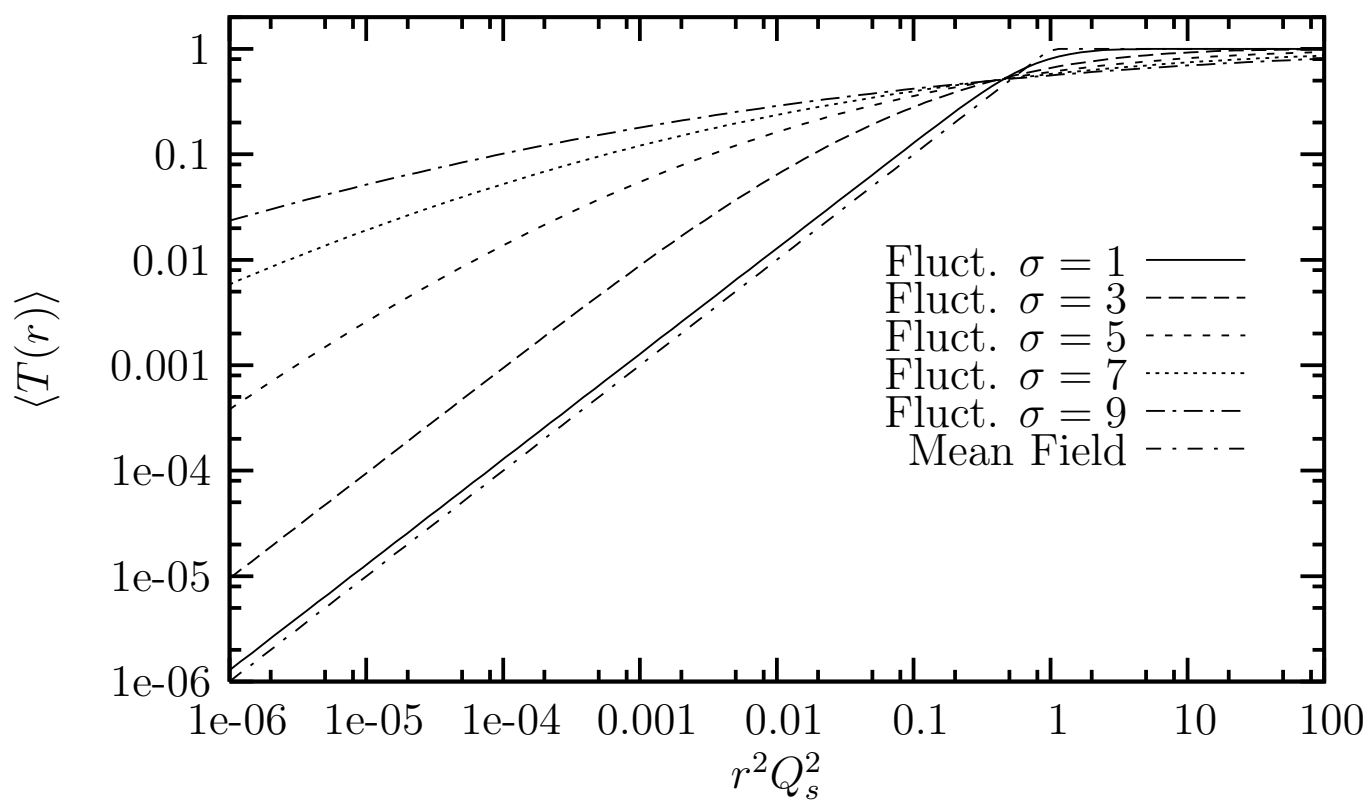

Fig. 10. The average dipole-hadron forward amplitude of Eq. (3.17) (with $\gamma=1$ ) as a function of the "standard" scaling variable $r^{2}\left\langle Q_{s}^{2}\right\rangle$ and for various values of the front dispersion $\sigma$.

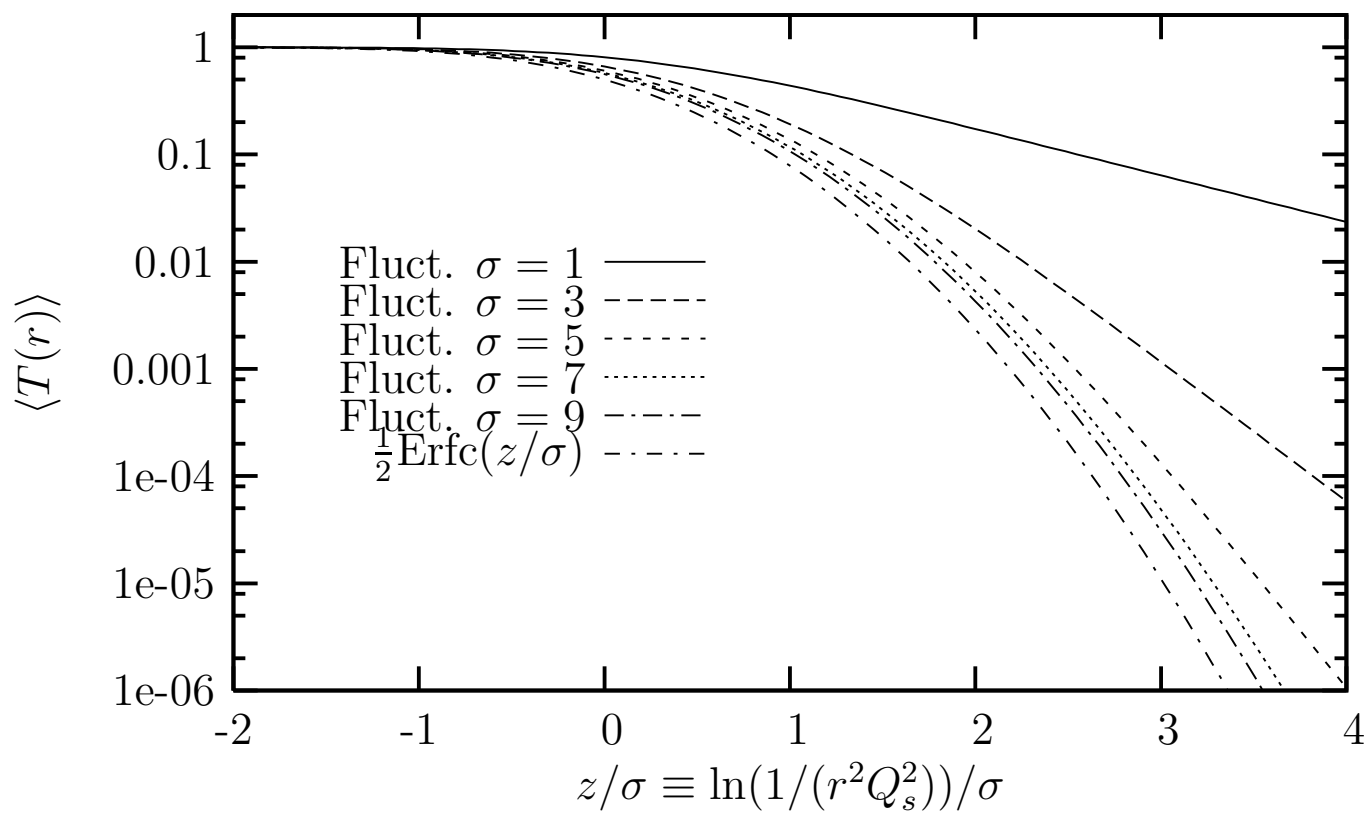

Fig. 11. The same as in Fig. 10 as a function of the "diffusive" scaling variable $z=-\ln \left(r^{2}\left\langle Q_{s}^{2}\right\rangle\right) / \sigma$. As $\sigma$ increases the amplitude approaches the limiting behavior in Eq. (3.13).

average amplitude decreases as a Gaussian in $z=\rho-\left\langle\rho_{s}\right\rangle$, and not as an exponential (as it happens for the single-event front within the geometric scaling window).

This behaviour is illustrated in Figs. 10 and 11, which show the average amplitude in Eq. (3.17) for $\gamma=1$ and various values of $\sigma$, as a function of either the 'geometric scaling' variable $r^{2}\left\langle Q_{s}^{2}\right\rangle$ (in Fig. 10), or the 'diffusive scaling' variable $z=\ln \left(1 / r^{2}\left\langle Q_{s}^{2}\right\rangle\right) / \sigma$ (in Fig. 11). The "mean field" curve in Fig. 10 represents the limit $\sigma \rightarrow 0$, that is, the single-event 
profile in Eq. (3.16). This shows geometric scaling, and would be the actual behaviour in the absence of fluctuations. As manifest on Figs. 10 and 11, when increasing $\sigma$, the average amplitude deviates more and more from geometric scaling and eventually approaches the limiting profile in Eq. (3.13) (displayed as the "Erfc" curve in Fig. 11), which shows diffusive scaling.

These considerations show that the mean field approximations (like the BK equation) completely fail to describe the dipole scattering at high energy. This is particularly striking when one considers multi-dipole correlations : in the high-energy regime (3.11) these correlations are given by Eq. (3.14), which shows that the mean-field factorization (2.32) is maximally violated. For instance, within the range $\sigma \ll z \ll \sigma^{2}$, the average amplitudes are weak, yet strongly correlated :

$$
\langle T(\rho)\rangle \simeq\left\langle T^{2}(\rho)\right\rangle \simeq\left\langle T^{N}(\rho)\right\rangle \approx \frac{1}{2 \sqrt{\pi}} \frac{\sigma}{z} \mathrm{e}^{-z^{2} / \sigma^{2}} \quad \text { for } \quad \sigma \ll z \ll \sigma^{2} .
$$

Accordingly, the $N$-dipole amplitudes with enter the evolution equations with Pomeron loops become as important as the one-dipole amplitude $\langle T(\rho)\rangle_{Y}$ already in the weak scattering regime, and not only in the region where the unitarity corrections are important. This is so because the individual fronts which contribute to these average amplitudes are themselves at saturation, and thus they are sensitive to unitarity corrections in the eventby-event description.

This dominance of saturated gluon configuration within the diffusive scaling region has another interesting consequence, to which we shall refer as the rigidity of the average amplitude: This is the property that, within the intermediate weak scattering regime at $\sigma \ll z \ll \sigma^{2}$, the average amplitude increases unusually slow when increasing $Y$ at fixed dipole size (i.e., at fixed $\rho$ ). To better appreciate this property, recall first the rapid, BFKL, increase of the average amplitude in the weak scattering regime at low and intermediate energies, say, in the geometric scaling window (cf. Eqs. (1.1)):

$$
\frac{\partial\langle T(r)\rangle_{Y}}{\partial Y} \sim \gamma_{0} \lambda \bar{\alpha}_{s}\langle T(r)\rangle_{Y} \quad \text { when } \quad r^{2}\left\langle Q_{s}^{2}\right\rangle \ll 1
$$

On the other hand, Eq. (3.13) implies

$$
\begin{aligned}
\frac{\partial\langle T(r)\rangle_{Y}}{\partial Y} & =\frac{1}{2} \operatorname{Erfc}^{\prime}\left(\frac{z}{\sigma}\right)\left(\frac{1}{\sigma} \frac{\mathrm{d} z}{\mathrm{~d} Y}-\frac{z}{\sigma^{2}} \frac{\mathrm{d} \sigma}{\mathrm{d} Y}\right) \\
& =\bar{\alpha}_{s}\left(\lambda+\frac{D_{\mathrm{fr}}}{2} \frac{z}{\sigma^{2}}\right) \frac{1}{\sqrt{\pi} \sigma} \exp \left(-\frac{z^{2}}{\sigma^{2}}\right) \sim \lambda \bar{\alpha}_{s} \frac{z}{\sigma^{2}}\langle T(r)\rangle_{Y}
\end{aligned}
$$

where we have used $z=\rho-\left\langle\rho_{s}\right\rangle,\left\langle\rho_{s}\right\rangle \simeq \lambda \bar{\alpha}_{s} Y$ and $\sigma^{2} \simeq D_{\text {fr }} \bar{\alpha}_{s} Y$, and the last estimate holds for $\sigma \ll z \ll \sigma^{2}$ (compare to Eq. (3.24)). Clearly, as compared to the intermediate energy (or mean-field) regime in Eq. (3.25), the relative variation of the high-energy amplitude in Eq. (3.26) is smaller by a factor $z / \sigma^{2} \ll 1$. This is so since the individual fronts which contribute to $\langle T(r)\rangle_{Y}$ in this regime are all at saturation, so by themselves they cannot increase when further increasing $Y$; the comparatively slow growth visible in Eq. (3.26) is rather due to the progression of the average front towards larger values of $\rho$. 


\section{Fluctuation effects on deep inelastic scattering at high energy}

In this section, we shall consider the high-energy limit of the inclusive and diffractive cross-sections introduced in Sect. 2, with the purpose of demonstrating the fundamental change of behaviour introduced in this regime by the gluon-number fluctuations. On this occasion, we shall present the first calculation of the DIS cross-sections including the effects of the Pomeron loops, and we shall compare our results with the corresponding predictions of the mean field approximation (the BK equation).

\subsection{The general set-up}

To perform this analysis, we need to complete our previous factorization formulæ with the electromagnetic vertex describing the dissociation of the virtual photon into a $q \bar{q}$ pair. Within the present, high-energy, approximations, this vertex factorizes out and the DIS cross-sections for the (inclusive and diffractive) $\gamma^{*} h$ scattering can be computed as $[70,71]$

$$
\frac{\mathrm{d} \sigma_{\text {tot }}^{\gamma}}{\mathrm{d}^{2} b}\left(Y, Q^{2}\right)=\int_{0}^{1} \mathrm{~d} v \int \mathrm{d}^{2} \boldsymbol{r} \sum_{\alpha=L, T}\left|\psi_{\alpha}^{\gamma}(v, r ; Q)\right|^{2} P_{\text {tot }}(\boldsymbol{b}, \boldsymbol{r} ; Y),
$$

and, respectively (recall that $Y_{\text {gap }}$ denotes the minimal rapidity gap),

$$
\frac{\mathrm{d} \sigma_{\text {diff }}^{\gamma}}{\mathrm{d}^{2} b}\left(Y, Y_{\text {gap }}, Q^{2}\right)=\int_{0}^{1} \mathrm{~d} v \int \mathrm{d}^{2} \boldsymbol{r} \sum_{\alpha=L, T}\left|\psi_{\alpha}^{\gamma}(v, r ; Q)\right|^{2} P_{\text {diff }}\left(\boldsymbol{b}, \boldsymbol{r} ; Y, Y_{\text {gap }}\right) .
$$

In these equations, $\left|\psi_{T / L}^{\gamma}\right|^{2}$ are probability densities for the $q \bar{q}$ dissociation of a virtual photon with either transverse $(T)$ or longitudinal $(L)$ polarization (these can be found in the literature and are displayed, for convenience, in Appendix A), $\boldsymbol{r}=\boldsymbol{x}-\boldsymbol{y}$ and $\boldsymbol{b}=(\boldsymbol{x}+\boldsymbol{y}) / 2$ are the transverse size and the impact parameter of the $q \bar{q}$ pair, $r \equiv|\boldsymbol{r}|$, $v$ is the fraction of the photon longitudinal momentum taken away by the quark, and $P_{\text {tot }}$ and $P_{\text {diff }}$ are the total and, respectively, diffractive probabilities for onium-hadron scattering, as introduced in Eqs. (2.3) and (2.11).

The above formulæ are a priori frame-independent, but in order to be able to use our expression (2.3) for $P_{\text {diff }}$, we need to evaluate the diffractive cross-section (4.2) in the frame where $Y_{0}=Y_{\text {gap }}$. On the other hand, the inclusive cross-section is most simply evaluated in the frame where $Y_{0}=Y$ and the projectile is an elementary $q \bar{q}$ pair:

$$
\frac{\mathrm{d} \sigma_{\text {tot }}^{\gamma}}{\mathrm{d}^{2} b}=\int_{0}^{1} \mathrm{~d} v \int \mathrm{d}^{2} \boldsymbol{r} \sum_{\alpha=L, T}\left|\psi_{\alpha}^{\gamma}(v, r ; Q)\right|^{2} 2 \operatorname{Re}\langle T(\boldsymbol{b}, \boldsymbol{r})\rangle_{Y} .
$$

The factorization formula (4.2) holds within the same approximations as previously used in the calculation of $P_{\text {diff }}$, namely, the leading logarithmic approximations with respect to both $\ln \left(1 / x_{\mathbb{P}}\right) \equiv Y_{\text {gap }}$ and $\ln (1 / \beta) \equiv Y-Y_{\text {gap }}$. Under these assumptions, the diffractive cross-section depends upon $x_{\mathbb{P}}$ and $\beta$ only via the high-energy evolutions of the target and the projectile, respectively. In the high-energy regime of interest, these approximations 
are certainly correct in so far as the $x_{\mathbb{P}}$-dependence is concerned (since $x_{\mathbb{P}}$ is truly small), but they fail to describe the actual $\beta$-dependence in the vicinity of $\beta=1$ (i.e., in the case of a relatively small mass $M_{X}^{2} \lesssim Q^{2}$ for the diffractive system). In fact, within the present approximations, the diffractive cross-section for $\beta \simeq 1$ reduces to its 'elastic' piece in Eqs. (2.1) and (2.2), which is independent of $\beta$. However, for $\beta \simeq 1$, the projectile is simply a $q \bar{q}$ pair, and for that pair the generalization of Eq. (4.2) which provides the correct $\beta$-dependence is known $[36,37]$ and will be exhibited in Appendix B (see Eq. (B.1)). It is therefore quite straightforward to improve the subsequent analysis in the vicinity of $\beta=1$, whenever this is needed. However, since our main interest here is rather in the high-energy limit $x_{\mathbb{P}} \rightarrow 0$ at fixed $\beta$, we find it more convenient to focus on generic values of $\beta \ll 1$, for which the relatively simple factorization formula (4.2) applies as written.

By using $P_{\text {diff }}=P_{\text {el }}+P_{\text {diff }}^{\text {inel }}$, cf. Eq. (2.18), it is further possible to separate the 'elastic' and 'inelastic' components of the diffractive cross-section (4.2), as shown in Eq. (2.1). Although these components cannot be separately measured in DIS, as explained at the end of Sect. 2.1, this decomposition is still useful because, as we shall argue below, the elastic component dominates at high energy, up to relatively large values of $Q^{2}$ (within the region for diffusive scaling). Namely, we shall find that within the high-energy region defined by Eq. (3.11), the diffractive probability is predominantly elastic:

$$
P_{\text {diff }}\left(r ; Y, Y_{\text {gap }}\right) \simeq P_{\text {el }}(r ; Y) \quad \text { when } \quad \sigma \gamma_{0} \gg 1 \text { and } \quad \ln \frac{1}{r^{2}\left\langle Q_{s}^{2}\right\rangle} \ll \sigma^{2}
$$

Since, moreover, the convolutions with the virtual photon wavefunction in Eqs. (4.1) and (4.2) are dominated by dipole sizes $r \sim 1 / Q$ within the whole kinematical region of interest (this will be explicitly checked below), the dominance of the elastic scattering extends to the diffractive cross-section, as anticipated :

$$
\frac{\mathrm{d} \sigma_{\mathrm{diff}}^{\gamma}}{\mathrm{d}^{2} b}\left(Y, Y_{\text {gap }}, Q^{2}\right) \simeq \frac{\mathrm{d} \sigma_{\mathrm{el}}^{\gamma}}{\mathrm{d}^{2} b}\left(Y, Q^{2}\right) \quad \text { when } \quad \sigma \gamma_{0} \gg 1 \quad \text { and } \quad \ln \frac{Q^{2}}{\left\langle Q_{s}^{2}\right\rangle} \ll \sigma^{2}
$$

This in turn implies that, within the present approximations at high energy, $\sigma_{\text {diff }}$ is quasiindependent of the minimal rapidity gap $Y_{\text {gap }}$ and can be simply computed as the elastic scattering of the elementary $q \bar{q}$ pair in the frame where $Y_{0}=Y$ (cf. Eq. (2.10)):

$$
\frac{\mathrm{d} \sigma_{\mathrm{el}}^{\gamma}}{\mathrm{d}^{2} b}=\int_{0}^{1} \mathrm{~d} v \int \mathrm{d}^{2} \boldsymbol{r} \sum_{\alpha=L, T}\left|\psi_{\alpha}^{\gamma}(v, r ; Q)\right|^{2}\left|\langle T(\boldsymbol{b}, \boldsymbol{r})\rangle_{Y}\right|^{2}
$$

In view of this and of Eq. (4.3), we conclude that, for sufficiently high energy and up to relatively large $Q^{2} \gg\left\langle Q_{s}^{2}\right\rangle$ (cf. Eq. (4.5)), the calculation of both the inclusive and the diffractive cross-sections requires merely the knowledge of the forward amplitude $\langle T(\boldsymbol{b}, \boldsymbol{r})\rangle_{Y}$ for the $q \bar{q}$ pair in the frame where the target carries most of the total energy.

The dominance of the elastic over the inelastic scattering in diffractive DIS at large $Q^{2}$ and relatively small $\beta$ is an essential consequence of fluctuations - in the mean field approximation, the opposite situation holds ! $[40,50]$ — , and it might even seem counterintuitive at a first sight: At small $\beta$, the projectile contains many gluons, yet Eq. (4.4) implies that the relative normalization of these many Fock states remains unchanged after 
the collision. But in view of the discussion in Sect. 3, this property is in fact natural, as we explain now:

Note first that Eq. (4.4) becomes natural in the saturation regime at $r^{2}\left\langle Q_{s}^{2}\right\rangle \gg 1$, where $P_{\text {diff }}$ and $P_{\text {el }}$ attain their respective unitarity bounds: $P_{\text {diff }}=P_{\text {el }}=1$. In that regime, all the Fock states in the projectile are equally absorbed, since they are completely absorbed. Thus, for low $Q^{2} \lesssim\left\langle Q_{s}^{2}\right\rangle$ at least, the inelastic diffraction is naturally suppressed. Now, in the discussion in Sect. 3 we have noticed the tendency of the fluctuations to 'pushup the saturation physics', that is, to extend a behaviour which looks natural in the saturation regime up to relatively large values of $Q^{2}$, within a distance $\ln \left(Q^{2} /\left\langle Q_{s}^{2}\right\rangle\right) \sim \sigma^{2}$ above the average saturation momentum which is fixed by the front dispersion. Physically, this is so since, within the relevant region at large $Q^{2}$ (the 'diffusive scaling' region), the cross-section is controlled by 'black spots' in the target wavefunction, i.e., by rare gluon configurations which are already at saturation. Hence, in any scattering event which contributes significantly to the diffractive probability (2.3), the projectile with its all Fock space components is completely absorbed - the diffraction is purely elastic ! - , and the reason where $P_{\text {diff }}$ is nevertheless smaller than one is just because the probability to find such a 'dense spot' is relatively small when $Q^{2} \gg\left\langle Q_{s}^{2}\right\rangle$.

Still from the discussion in Sect. 3, we expect the inelastic probability $P_{\text {diff }}^{\text {inel }}$ to be suppressed with respect to the elastic one $P_{\text {el }}$ by powers of $1 / \sigma$ and/or $z / \sigma^{2}$, and this will be indeed confirmed by the subsequent calculations. But although suppressed in so far as the contribution to the cross-section (4.2) - which, we recall, is 'inclusive' with respect to the rapidity gap (in the sense of including all the gap values from $Y_{\text {gap }}$ to $Y$ ) - is concerned, the inelastic diffraction represents the leading contribution to the differential cross-section per unit rapidity gap at sufficiently small $\beta \ll 1$, and hence cannot be ignored when computing this particular quantity (even at very high energy). In fact, within the present approximations, the contribution of the elastic scattering to the differential cross-section $\mathrm{d} \sigma_{\text {diff }} / \mathrm{d} \ln (1 / \beta)$ is a $\delta$-function peaked at $\beta=1$. This contribution gets smeared when going beyond the leading-log approximation with respect to $\ln (1 / \beta)$ (see Eq. (B.1)), but is in any case localized near $\beta=1$; hence, for $\beta \ll 1$ we expect most of the respective contribution to come from the inelastic diffraction:

$$
\frac{\mathrm{d} \sigma_{\text {diff }}^{\gamma}}{\mathrm{d}^{2} b \mathrm{~d} \ln (1 / \beta)} \simeq \int_{0}^{1} \mathrm{~d} z \int \mathrm{d}^{2} \boldsymbol{r} \sum_{\alpha}\left|\psi_{\alpha}^{\gamma}(z, r ; Q)\right|^{2}\left(-\frac{\partial P_{\text {diff }}^{\text {inel }}}{\partial Y_{\text {gap }}}\left(\boldsymbol{b}, \boldsymbol{r} ; Y, Y_{\text {gap }}\right)\right) \quad \text { for } \quad \beta \ll 1,
$$

where in the r.h.s. $Y_{\text {gap }} \equiv Y-\ln (1 / \beta)$.

To summarize, within the high-energy regime specified in Eq. (4.5), the diffractive crosssection (4.2) is dominated by its elastic component and can be computed with the $q \bar{q}$ pair alone (cf. Eqs. (4.5)-(4.6)), so like the inclusive cross-section (cf. Eq. (4.3)). But the calculation of the inelastic diffraction is still necessary, for two reasons: (i) to explicitly check the dominance of the elastic over the inelastic diffraction, and (ii) to compute the differential diffractive cross-section (4.7) at small $\beta \ll 1$. Within the present approximations, $P_{\text {diff }}^{\text {inel }}$ is given by Eqs. (2.18) and (2.3) which, we recall, are valid so long as the saturation effects in the projectile wavefunction remain negligible (this requires $\bar{\alpha}_{s} \ln (1 / \beta) \ll \ln \left(1 / \alpha_{s}^{2}\right)$, cf. Eq. (2.13)). For any $\beta$ satisfying this condition, Eq. (2.3) can 
be in principle computed by using the dipole probabilities $P_{N}$ obtained from Monte-Carlo simulations of the dipole picture [11] together with the dipole scattering amplitudes constructed in Sect. 3 (or, more generally, obtained by numerically solving the Pomeron loop equations [14]).

But in order to illustrate the main points of physics, it is preferable to use analytic calculations, and this is what we shall do in what follows. For these calculations to be tractable, we shall restrict our computation of $P_{\text {diff }}^{\text {inel }}$ to the simplest non-trivial case - that where the onium wavefunction at the time of scattering contains only two components: a one-dipole state representing the original $q \bar{q}$ pair, and a two-dipole state corresponding to a $q \bar{q} g$ configuration. This is the physical situation in a frame in which the rapidity $Y-Y_{0}=\ln (1 / \beta)$ of the projectile is relatively large, so its high-energy evolution cannot be neglected, but not too large, so that one can restrict oneself to a single step in this evolution: the emission of one gluon with longitudinal momentum fraction $\beta$. This restriction on $\beta$ does not entail any serious loss of generality in so far as the proof of Eq. (4.5) is concerned - as previously explained, the dominance of elastic over inelastic diffraction at high energy follows from general arguments, and our subsequent calculation is merely intended to illustrate such arguments and to render them more quantitative. On the other hand, this restriction would become of problem for phenomenological studies of diffraction at very small values of $\beta$; but in that case, it should be straightforward - at least, at conceptual level — to extend our analysis by using numerical simulations of the dipole picture within Eq. (2.3). Note that the $q \bar{q}$ and the $q \bar{q} g$ components have been already included in previous studies of DIS in the presence of saturation [36, 37, 39-43, 45-47, 49,50,52-54], which were however based on the mean field approximation for the target correlations. The comparison between such previous studies in the literature and our subsequent results will make it easier to emphasize the role of the gluon-number fluctuations at high energy.

To conclude this subsection, let us summarize here, for more clarity, the strategy that we shall adopt in our analysis: In what follows, we shall evaluate the cross-sections in Eqs. (4.3), (4.6) and (4.7) (the latter, at the level of the $q \bar{q} g$ Fock state) by using the highenergy estimates (3.13)-(3.14) for the dipole amplitudes. Note that, whereas the functional form of these amplitudes is universal (in the sense explained in the Introduction), the coefficients $\lambda$ and $D_{\text {fr }}$ which implicitly enter their expressions (via the $Y$-dependencies of $\left\langle\rho_{s}\right\rangle$ and $\sigma^{2}$ ) are not: they depend upon the details of the QCD evolution, and presently they are not well under control, except in the limit $\alpha_{s} \rightarrow 0$ (cf. Sect. 3.2). To avoid specifying these parameters, we shall perform our subsequent analysis in terms of the variables $\rho-\left\langle\rho_{s}\right\rangle$ and $\sigma$. That is, we shall increase the energy by increasing the value of $\sigma$, and we shall measure all dimensionful quantities, like $r^{2}$ or $Q^{2}$, in units of $\left\langle Q_{s}^{2}\right\rangle$. This strategy is physically meaningful, since the proper way to approach the "high-energy limit" is to simultaneously increase $Y$ and $Q^{2}$, in such a way that the ratio $Q^{2} /\left\langle Q_{s}^{2}\right\rangle$ remains within the interesting scaling region in Fig. 1.

To better emphasize the effects of the fluctuations, we shall also estimate the relevant convolutions with the following 'mean-field' dipole amplitude

$$
\bar{T}(r, Y)=\left\{\begin{array}{cc}
\left(r^{2} Q_{s}^{2}(Y)\right)^{\gamma} & \text { for } r \leq 1 / Q_{s}(Y) \\
1 & \text { for } r>1 / Q_{s}(Y)
\end{array}\right.
$$


which is representative for either the Golec-Biernat and Wüsthoff (GBW) 'saturation model' [40] (in that case, $\gamma=1$ ), or for the solution to the BK equation in the regime of geometric scaling (when $\gamma=\gamma_{0} \approx 0.63$ ). More generally, for our present purposes, the amplitude (4.8) is also representative for the actual situation in QCD at intermediate energies, that is, so long as the dispersion of the fronts remains negligible (cf. Eq. (3.20)). To study the transition between this regime and the high-energy regime defined by Eq. (3.11), where the amplitude takes its "error-function" form in Eq. (3.13), we shall perform numerical simulations with the dipole amplitude in Eq. (3.17). Indeed, the latter provides a smooth interpolation between the mean-field amplitude (4.8) at low energy $(\sigma \rightarrow 0)$ and the error-function amplitude (3.13) at high energy $(\sigma \rightarrow \infty)$. In most of the explicit calculations below, we shall choose $\gamma=1$, for simplicity.

To obtain order-of-magnitude estimates in what follows, we shall rely on simplified versions of the convolutions appearing in Eqs. (4.1)-(4.2), which in addition to being simpler, have also the merit to clearly separate the physical origin of the various contributions. The relevant simplifications exploit the properties of the "virtual photon wavefunctions" $\left|\psi_{T / L}^{\gamma}\right|^{2}$, as explained in the Appendix A. The ensuing, simplified, formulæ read then

$$
\int \mathrm{d} v \mathrm{~d}^{2} \boldsymbol{r}\left|\psi_{T}^{\gamma}(v, r ; Q)\right|^{2} f(r) \sim \alpha_{\mathrm{em}} N_{c} \sum_{f} e_{f}^{2}\left[\int_{0}^{2 / Q} \frac{\mathrm{d} r}{r} f(r)+\frac{1}{Q^{2}} \int_{2 / Q}^{\infty} \frac{\mathrm{d} r}{r^{3}} f(r)\right],
$$

and, respectively,

$$
\int \mathrm{d} v \mathrm{~d}^{2} \boldsymbol{r}\left|\psi_{L}^{\gamma}(v, r ; Q)\right|^{2} f(r) \sim \alpha_{\mathrm{em}} N_{c} \sum_{f} e_{f}^{2}\left[Q^{2} \int_{0}^{2 / Q} \mathrm{~d} r r f(r)+\frac{1}{Q^{4}} \int_{2 / Q}^{\infty} \frac{\mathrm{d} r}{r^{5}} f(r)\right]
$$

where in writing the r.h.s.'s we have kept the various parametric dependencies, but ignored all numerical factors. For each of these expressions, the first term within the square brackets corresponds to the symmetric configurations, for which $v \sim 1 / 2$ (in such configurations, the longitudinal momentum of the incoming photon is "democratically" divided among the quark and the antiquark), and the second one, to the aligned jet configurations, for which $v$ is either close to zero, or close to one (either the quark, or the antiquark, carries most of the total longitudinal momentum).

\subsection{The $q \bar{q}$ component}

In this subsection, we shall evaluate the DIS cross-sections which can be computed from the scattering of the $q \bar{q}$ alone, namely, the inclusive cross-section (4.3) and the elastic component of the diffractive cross-section, Eq. (4.6). As anticipated in the previous discussion, Eq. (4.6) is in fact the dominant contribution to the diffractive cross-section (4.2) at high energy. Accordingly, the cross-sections that we shall compute in this subsection represent our main results in this paper.

To render the subsequent discussion more intuitive, it is instructive to see the plots of the integrands in Eqs. (4.3) and (4.6), as numerically computed with the two limiting expres- 

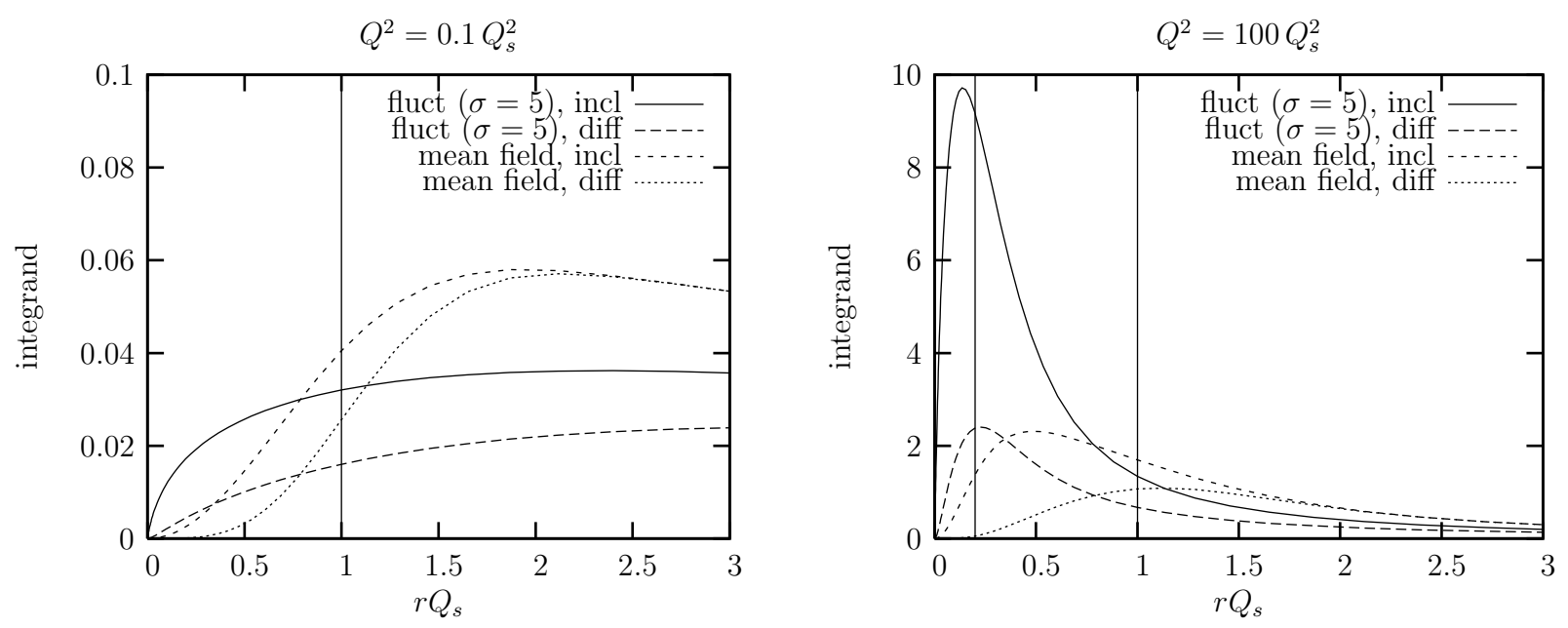

Fig. 12. The $q \bar{q}$ contributions to the integrands in Eqs. (4.3) and (4.6) as computed with two different expressions for the dipole amplitude - the mean-field amplitude (4.8) with $\gamma=1$ and the 'fluctuation' amplitude (3.13) - are plotted as functions of $r Q_{s}$ for two values of $Q^{2}$ : $Q^{2}=0.1 Q_{s}^{2}$ (left) and $Q^{2}=100 Q_{s}^{2}$ (right).

sions for the dipole amplitude: the high-energy approximation (3.13) (the 'fluctuation' piece in the plots in Fig. 12) and the mean-field model of Eq. (4.8) (with $\gamma=1$ for definiteness). As manifest on Fig. 12, and it will be analytically demonstrated in what follows, the strength of the integration is shifted towards smaller dipoles sizes after including the effects of fluctuations. This phenomenon becomes truly spectacular at high $Q^{2}$, where the integrand corresponding to 'fluctuations' appears to be strongly peaked at $r \sim 2 / Q$ (for both inclusive and diffractive cross-sections), while that for the mean field approximation is rather smoothly distributed at all sizes $r \gtrsim 2 / Q$ (with only mild maxima at $r \sim 2 / Q$ for $\sigma_{\text {tot }}$ and, respectively, at $r \sim 1 / Q_{s}$ for $\left.\sigma_{\text {diff }}\right)$.

\subsubsection{The mean-field approximation}

Let us first present the corresponding mean-field estimates, which are well-known (see, e.g., Refs. [39, 40, 54]), but are interesting here as a term of comparison with the forthcoming results which will include the effects of fluctuations. These estimates will be obtained here by using the dipole amplitude (4.8) together with the approximations in Eqs. (4.9)(4.10). To avoid a proliferation of physical situations, we shall focus on the case $Q^{2} \gg Q_{s}^{2}$, where the contrast with the high energy behaviour turns out to be most important. For that case, we shall distinguish between the total and the diffractive cross-sections, and also between transverse and longitudinal polarizations for $\gamma^{*}$. To simplify writing, we shall omit the overall factor $\alpha_{\mathrm{em}} N_{c} \sum_{f} e_{f}^{2}$, which is common to all the cross-sections.

\section{i) Total cross-sections}

Choosing $\gamma=1$ in Eq. (4.8), the transverse contribution to Eq. (4.3) is found as

$$
\frac{\mathrm{d} \sigma_{\mathrm{tot}}^{T}}{\mathrm{~d}^{2} b} \sim \int_{0}^{2 / Q} \frac{\mathrm{d} r}{r} r^{2} Q_{s}^{2}+\frac{1}{Q^{2}} \int_{2 / Q}^{1 / Q_{s}} \frac{\mathrm{d} r}{r^{3}} r^{2} Q_{s}^{2}+\frac{1}{Q^{2}} \int_{1 / Q_{s}}^{\infty} \frac{\mathrm{d} r}{r^{3}} \sim \frac{Q_{s}^{2}}{Q^{2}} \ln \left(\frac{Q^{2}}{Q_{s}^{2}}\right)
$$


where the dominant term, as isolated in the r.h.s., comes from the aligned-jet configurations with dipole sizes $r$ within the range $2 / Q<r<1 / Q_{s}$. For the longitudinal sector, one similarly obtains

$$
\frac{\mathrm{d} \sigma_{\mathrm{tot}}^{L}}{\mathrm{~d}^{2} b} \sim Q^{2} \int_{0}^{2 / Q} \mathrm{~d} r r^{3} Q_{s}^{2}+\frac{1}{Q^{4}} \int_{2 / Q}^{1 / Q_{s}} \frac{\mathrm{d} r}{r^{3}} Q_{s}^{2}+\frac{1}{Q^{4}} \int_{1 / Q_{s}}^{\infty} \frac{\mathrm{d} r}{r^{5}} \sim \frac{Q_{s}^{2}}{Q^{2}}
$$

where the leading-order term comes from dipole sizes $r \sim 1 / Q$, and it receives equally important contributions from both the symmetric and the aligned-jet configurations.

The above results show that, at sufficiently large $Q^{2}$, the transverse sector dominates over the longitudinal one, by a $\operatorname{logarithm} \ln \left(Q^{2} / Q_{s}^{2}\right)$. However, this dominance disappears at lower values of $Q^{2}$, within the geometric scaling window in Fig. 1. Indeed, in that regime one must rather use $\gamma=\gamma_{0} \approx 0.63$, and then one finds that both $\sigma_{\text {tot }}^{T}$ and $\sigma_{\text {tot }}^{L}$ are of the same order, namely $\sim\left(Q_{s}^{2} / Q^{2}\right)^{\gamma_{0}}$, and they are dominated by dipole sizes $r \sim 1 / Q$.

After similarly treating the case $Q^{2} \ll Q_{s}^{2}$, one finds the following limiting behaviours for the total $\gamma^{*} h$ cross-section in this mean-field scenario :

$$
\frac{\mathrm{d} \sigma_{\text {tot }}^{\gamma}}{\mathrm{d}^{2} b} \approx \begin{cases}\ln \left(Q_{s}^{2} / Q^{2}\right) & \text { for } Q^{2} \ll Q_{s}^{2} \\ Q_{s}^{2} / Q^{2} \ln \left(Q^{2} / Q_{s}^{2}\right) & \text { for } Q^{2} \gg Q_{s}^{2}\end{cases}
$$

\section{ii) Diffractive cross-sections}

The diffractive cross-section (4.6) involves the dipole amplitude squared, and this leads to important differences with respect to the inclusive cross-section, at least in this meanfield scenario. The contribution of the small dipoles is now strongly suppressed, because $\bar{T}^{2} \simeq\left(r^{2} Q_{s}^{2}\right)^{2 \gamma}$ for $r \ll 1 / Q_{s}$. Accordingly, the dominant contribution to $\sigma_{\text {diff }}$ comes from relatively large dipole sizes, of the order of the saturation length: $r \sim 1 / Q_{s}[34,35,40,68]$.

To see this, let us focus on the contribution of the aligned-jet configurations within the transverse sector (this turns out to be the dominant piece). The relevant integral reads

$$
\frac{\mathrm{d} \sigma_{\mathrm{diff}}^{T}}{\mathrm{~d}^{2} b} \sim \frac{1}{Q^{2}} \int_{2 / Q}^{\infty} \frac{\mathrm{d} r}{r^{3}} \bar{T}^{2} \sim \frac{1}{Q^{2}} \int_{2 / Q}^{1 / Q_{s}} \frac{\mathrm{d} r}{r^{3}}\left(r^{2} Q_{s}^{2}\right)^{2 \gamma}+\frac{1}{Q^{2}} \int_{1 / Q_{s}}^{\infty} \frac{\mathrm{d} r}{r^{3}} \sim \frac{Q_{s}^{2}}{Q^{2}},
$$

where for more clarity we have split the integration domain into two: $2 / Q<r<1 / Q_{s}$ and $r>1 / Q_{s}$. It can be easily checked that, so long as $\gamma>1 / 2$, both domains contribute on equal footing to the leading order result $\sim Q_{s}^{2} / Q^{2}$, which is generated by dipole sizes $r \sim 1 / Q_{s}$, as anticipated. Indeed, the integral over the first domain is dominated by large values of $r$, of the order of the upper cutoff $1 / Q_{s}$, while that over the second domain is saturated by small values of $r$, close to the respective lower cutoff $1 / Q_{s}$. By comparison, the other contributions to diffraction - that of the symmetric transverse configurations, and the whole longitudinal contribution - are of 'higher-twist order', that is, they are suppressed by powers of $Q_{s}^{2} / Q^{2} \ll 1$ with respect to the leading contribution (4.14).

We thus arrive at the (by now) standard picture of DIS diffraction in the presence of 
saturation $[34,35,40]$, in which $\sigma_{\text {diff }}$ is a leading-twist quantity at high- $Q^{2}$, so like the inclusive cross-section (4.11). Accordingly, the ratio between the diffractive and the inclusive cross-sections is only slowly varying with $Q^{2}$ and with the total energy [40,47]

$$
R \equiv \frac{\left(\mathrm{d} \sigma_{\mathrm{diff}} / \mathrm{d}^{2} b\right)}{\left(\mathrm{d} \sigma_{\mathrm{tot}} / \mathrm{d}^{2} b\right)} \sim \frac{1}{\ln \left(Q^{2} / Q_{s}^{2}(Y)\right)}
$$

(this behaviour is illustrated by the 'mean-field' curve in Fig. 17), in rough agreement with the pattern observed in the corresponding HERA data at small $x$. This agreement has represented one of the main successes of the saturation models applied to the phenomenology at HERA [40,47].

Notice the qualitative difference between the ways that the inclusive and diffractive crosssections get constructed - $\sigma_{\text {tot }}$ receives leading-twist contributions from all dipole sizes, with a slight preference though for $r \sim 1 / Q$, whereas $\sigma_{\text {diff }}$ is dominated by dipoles with $r \sim 1 / Q_{s}$-, which nevertheless leads to quantitatively similar results (cf. Eq. (4.15)). For later reference, let us display here the $q \bar{q}$ contribution to the diffractive cross-section in the mean-field approximation for both small and large $Q^{2}$ :

$$
\frac{\mathrm{d} \sigma_{\mathrm{diff}}^{q \bar{q}}}{\mathrm{~d}^{2} b} \approx \begin{cases}\ln \left(Q_{s}^{2} / Q^{2}\right) & \text { for } Q^{2} \ll Q_{s}^{2} \\ Q_{s}^{2} / Q^{2} & \text { for } Q^{2} \gg Q_{s}^{2}\end{cases}
$$

The above results depend crucially upon the property of the mean-field amplitude $\bar{T}(r)$ to decrease very fast when decreasing $r$ below $1 / Q_{s}: \bar{T} \sim\left(r^{2} Q_{s}^{2}\right)^{\gamma}$ with $\gamma>1 / 2$. From the discussion in Sect. 3, we know that this behavior is eventually washed out by fluctuations when increasing energy. As we shall shortly see, this change of behaviour has dramatic consequences for the high-energy limit of both inclusive and diffractive DIS cross-sections.

\subsubsection{The high-energy behaviour: 'black spots' and diffusive scaling}

We now turn to the most interesting case for us here, namely the high-energy regime of Eq. (3.11). The kinematical region where the effects of the fluctuations are most visible, and that we shall concentrate on in what follows, is the high- $Q^{2}$ range defined by

$$
\sigma(Y) \gg 1 / \gamma_{0} \quad \text { and } \quad \sigma(Y) \ll Z \equiv \ln \frac{Q^{2}}{\left\langle Q_{s}^{2}\right\rangle} \ll \sigma^{2}(Y)
$$

For much smaller $Q^{2}$, such that $Q^{2} \ll Q_{s}^{2}(Y)$, the cross-sections are dominated by 'saturated' dipoles with sizes $r \gtrsim 1 / Q_{s}$, for which the mean field approximation works quite well. For much larger $Q^{2}$, such that $Z \gg \sigma^{2}(Y)$, one enters the standard perturbative regime where the amplitudes show color transparency and evolve according to the DGLAP equation.

We shall soon verify that, within the interesting range (4.17), the convolutions yielding the DIS cross-sections are dominated by dipole sizes $r \sim 1 / Q$ (at variance with the mean field case, especially in the case of diffraction). It is therefore appropriate to evaluate these convolutions with the expression (3.13) for the dipole amplitude, which is valid for 
$z \ll \sigma^{2}(Y)$. By using that, we shall be able to compute exactly the dominant behaviour of the cross-sections at high energy. But before describing the exact calculations, we shall present some parametric estimates which have the merit to simply demonstrate the physical points that we would like to emphasize here.

\section{i) Total cross-sections}

We start with the transverse sector and, for more clarity, we separate the respective contributions of the symmetric and the aligned-jet configurations:

$$
\frac{\mathrm{d} \sigma_{\text {tot }}^{T}}{\mathrm{~d}^{2} b}=S_{\text {tot }}^{T}+A_{\text {tot }}^{T},
$$

where (with $\left.z \equiv \ln \left(1 / r^{2}\left\langle Q_{s}^{2}\right\rangle\right)\right)$ :

$$
\begin{aligned}
& S_{\text {tot }}^{T} \sim \int_{0}^{2 / Q} \frac{\mathrm{d} r}{r}\langle T(r)\rangle_{Y} \sim \int_{Z}^{\infty} \mathrm{d} z \operatorname{Erfc}\left(\frac{z}{\sigma}\right), \\
& A_{\text {tot }}^{T} \sim \frac{1}{Q^{2}} \int_{2 / Q}^{\infty} \frac{\mathrm{d} r}{r^{3}}\langle T(r)\rangle_{Y} \sim \mathrm{e}^{-Z} \int_{-\infty}^{Z} \mathrm{~d} z \mathrm{e}^{z} \operatorname{Erfc}\left(\frac{z}{\sigma}\right) .
\end{aligned}
$$

Consider the large $Q^{2}$ case, such that $\sigma \ll Z \ll \sigma^{2}$. It is then easy to verify that: (a) the integrals giving $S_{\mathrm{tot}}^{T}$ and $A_{\mathrm{tot}}^{T}$ are dominated by their respective endpoints at $z=Z$ (i.e., by $r \sim 1 / Q$ ), (b) the dominant behaviour at large $Z$ is given by the Gaussian $\exp \left(-z^{2} / \sigma^{2}\right)$, and (c) after also computing the prefactors in front of this Gaussian, the dominant contribution is found to come from the symmetric configurations.

To demonstrate this, we shall first consider the symmetric integral:

$$
\begin{aligned}
S_{\text {tot }}^{T} & \sim \sigma \int_{Z / \sigma}^{\infty} \mathrm{d} x \operatorname{Erfc}(x) \sim \sigma \int_{Z / \sigma}^{\infty} \frac{\mathrm{d} x}{x} \mathrm{e}^{-x^{2}} \sim \sigma \int_{Z^{2} / \sigma^{2}}^{\infty} \frac{\mathrm{d} t}{t} \mathrm{e}^{-t} \\
& \sim \frac{\sigma^{3}}{Z^{2}} \exp \left(-\frac{Z^{2}}{\sigma^{2}}\right),
\end{aligned}
$$

where $x=z / \sigma, t=x^{2}$, and we have used the asymptotic behaviour of $\operatorname{Erfc}(x)$, cf. Eq. (3.18) (recall that $Z / \sigma \gg 1$ ). It is now clear that the integral over $t$ is dominated by its lower end at $t=Z^{2} / \sigma^{2}$, i.e., by $z=Z$, as anticipated.

The aligned-jet contribution can be similarly treated: because of the exponential factor $\mathrm{e}^{z}$ within the integrand, the integral in Eq. (4.20) is dominated by its upper cutoff at $z=Z$, which yields

$$
A_{\text {tot }}^{T} \sim \frac{\sigma}{Z} \exp \left(-\frac{Z^{2}}{\sigma^{2}}\right) .
$$

As anticipated, this contribution is suppressed (by a factor $Z / \sigma^{2} \ll 1$ ) with respect to the contribution (4.21) of the symmetric configurations. 
Similar conclusions hold for the longitudinal sector : the dominant contribution is again generated by dipole sizes $r \sim 1 / Q$ and it is of the same order as $A_{\text {tot }}^{T}$, Eq. (4.22), hence it is parametrically suppressed. We thus conclude that the inclusive cross-section in the highenergy limit and for relatively large $Q^{2}$ is dominated by symmetric dipole configurations with typical sizes $r \sim 1 / Q$ within the transverse sector.

It is in fact quite straightforward to go beyond the parametric estimate in Eq. (4.21) and compute the dominant behaviour exactly. We present here only the final results, since a similar calculation will be explained in more detail below, for the case of diffraction. Specifically, after also reintroducing the overall electromagnetic factor $F \equiv\left(N_{c} \alpha_{\mathrm{em}} / 2 \pi^{2}\right) \sum_{f} e_{f}^{2}$, one finds that the longitudinal part of the total cross-section is given by

$$
\frac{\mathrm{d} \sigma_{\text {tot }}^{L}}{\mathrm{~d}^{2} b} \simeq \frac{\pi F}{3} \operatorname{Erfc}\left(\frac{Z}{\sigma}\right)
$$

while the respective transverse part reads

$$
\frac{\mathrm{d} \sigma_{\mathrm{tot}}^{T}}{\mathrm{~d}^{2} b} \simeq \frac{\pi F}{3} \sigma \Phi_{1}\left(\frac{Z}{\sigma}\right)
$$

Here we have found convenient to define the function

$$
\Phi_{1}(x) \equiv \int_{x}^{\infty} \mathrm{d} v \operatorname{Erfc}(v)=\frac{1}{\sqrt{\pi}} \exp \left(-x^{2}\right)-x \operatorname{Erfc}(x)
$$

which has the following behavior in the various limits

$$
\Phi_{1}(x)= \begin{cases}2|x|+\frac{\exp \left(-x^{2}\right)}{2 \sqrt{\pi} x^{2}} & \text { for } x \ll-1 \\ \frac{1}{\sqrt{\pi}} & \text { for } x=0 \\ \frac{\exp \left(-x^{2}\right)}{2 \sqrt{\pi} x^{2}} & \text { for } x \gg 1 .\end{cases}
$$

Eqs. (4.23) and (4.24) hold for $Z \ll \sigma^{2}$ and are correct up to corrections of relative order $Z / \sigma^{2}$ and/or $1 / \sigma$. They confirm the previous estimates like (4.21) and imply the following, final, result for the total DIS cross-section in the high-energy regime:

$$
\frac{\mathrm{d} \sigma_{\mathrm{tot}}^{\gamma}}{\mathrm{d}^{2} b} \simeq \frac{\mathrm{d} \sigma_{\mathrm{tot}}^{T}}{\mathrm{~d}^{2} b} \simeq \frac{\pi F}{3} \sigma \Phi_{1}\left(\frac{\ln \left(Q^{2} /\left\langle Q_{s}^{2}\right\rangle\right)}{\sigma}\right) \quad \text { for } \sigma \gamma_{0} \gg 1 \text { and } \ln \frac{Q^{2}}{\left\langle Q_{s}^{2}\right\rangle} \ll \sigma^{2} .
$$

Note that the quantity $\left(\mathrm{d} \sigma_{\text {tot }}^{\gamma} / \mathrm{d}^{2} b\right) / \sigma$ shows diffusive scaling, i.e., it depends upon the kinematical variables $Q^{2}$ and $Y$ only via the dimensionless variable $Z / \sigma \equiv \ln \left(Q^{2} /\left\langle Q_{s}^{2}\right\rangle\right) / \sigma$.

Eq. (4.27) also allows us to study the behaviour of the cross-section at relatively low $Q^{2}$ (below and around $\left\langle Q_{s}^{2}\right\rangle$ ). Deeply inside the saturation region, one finds

$$
\frac{\mathrm{d} \sigma_{\text {tot }}^{\gamma}}{\mathrm{d}^{2} b} \approx \frac{2 \pi F}{3} \ln \frac{\left\langle Q_{s}^{2}\right\rangle}{Q^{2}} \quad \text { for } \quad \ln \left(\left\langle Q_{s}^{2}\right\rangle / Q^{2}\right) \gg \sigma
$$

which is in agreement, as expected, with the mean-field behaviour in Eq. (4.13). However, the difference with respect to the mean field situation occurs already in the vicinity of 


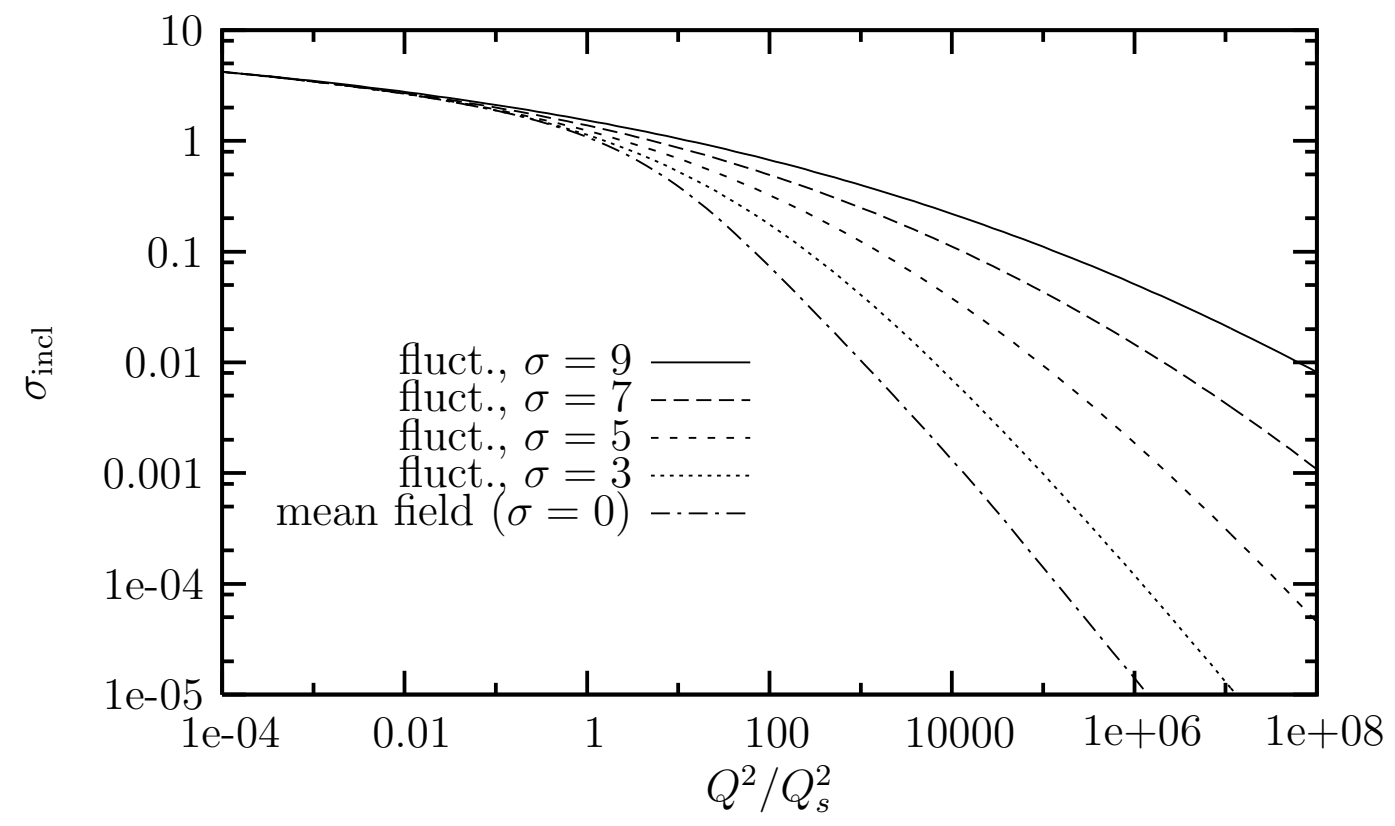

Fig. 13. The $\gamma^{*} h$ total cross section as a function of $Q^{2} /\left\langle Q_{s}^{2}\right\rangle$ and for various values of the front dispersion $\sigma$.

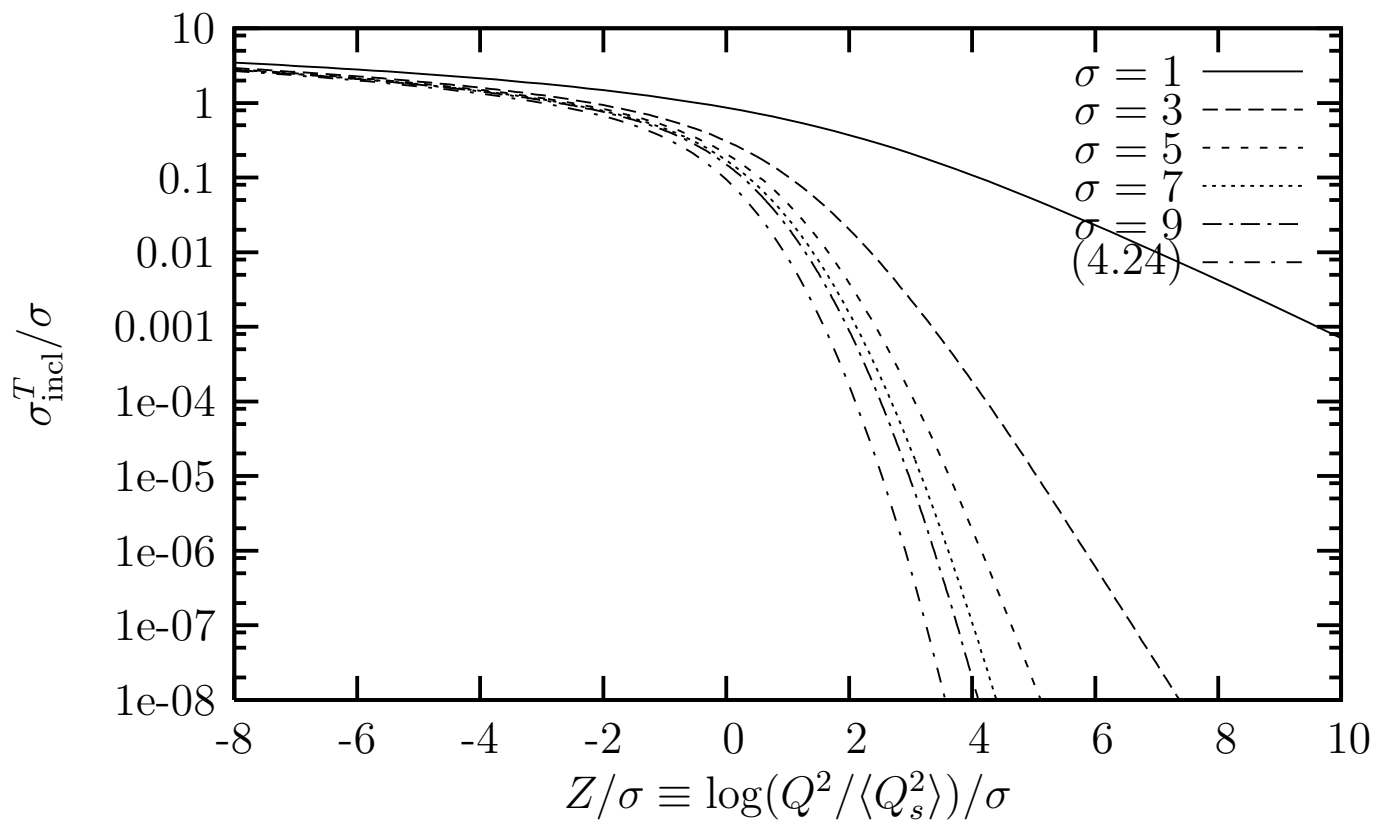

Fig. 14. The $\gamma^{*} h$ total cross section (divided by the front dispersion) as a function of the "diffusive" scaling variable $Z=\ln \left(Q^{2} /\left\langle Q_{s}^{2}\right\rangle\right) / \sigma$.

the (average) saturation line $Z=0$ : Within a wide interval $\left|\ln \left(Q^{2} /\left\langle Q_{s}^{2}\right\rangle\right)\right| \ll \sigma$ around this line, the cross-section (4.27) is rather large - since proportional to $\sigma$ - and $Q^{2}-$ independent:

$$
\frac{\mathrm{d} \sigma_{\mathrm{tot}}^{\gamma}}{\mathrm{d}^{2} b} \approx \frac{\sqrt{\pi} F}{3} \sigma \quad \text { for } \quad\left|\ln \left(Q^{2} /\left\langle Q_{s}^{2}\right\rangle\right)\right| \ll \sigma .
$$


The cross-section starts to fall only when $Q^{2}$ becomes so large that $\ln \left(Q^{2} /\left\langle Q_{s}^{2}\right\rangle\right) \gg \sigma$, and then it has a rapid fall, of the Gaussian type (cf. Eq. (4.21)).

This behaviour is illustrated in Figs. 13 and 14, which are the analog at the level of the DIS inclusive cross-section of Figs. 10 and 11 for the dipole amplitude. Namely, these figures represent results obtained via the numerical evaluation of the total cross-section (4.3) with the dipole amplitude in Eq. (3.17) with $\gamma=1$. Fig. 13 shows the increase in the deviation from the mean-field behaviour (and thus from geometric scaling) when increasing $\sigma$, whereas Fig. 14 demonstrates the emergence of the diffusive scaling and the convergence of the cross-section towards its high-energy asymptotic in Eq. (4.27).

\section{ii) Diffractive cross-sections}

Moving to the diffractive sector, one can immediately notice an important difference with respect to the mean-field scenario of Sect. 4.2.1: Whereas in that case, the replacement of the dipole amplitude by its square has strongly suppressed the small-dipole configurations and thus shifted the strength of the integration from $r \sim 1 / Q$ to $r \sim 1 / Q_{s}$, in the present, high-energy, case, there is no similar suppression, because both the Gaussian $\exp \left(-z^{2} / \sigma^{2}\right)$ and its square $\exp \left(-2 z^{2} / \sigma^{2}\right)$ decay rather slowly (as compared to the exponential $\mathrm{e}^{-z}$ ) within the interesting range at $\sigma \ll z \ll \sigma^{2}$. Accordingly, the aligned-jet contribution to high-energy $q \bar{q}$ diffraction, namely (compare to Eq. (4.14))

$$
A_{\mathrm{diff}}^{T} \sim \frac{1}{Q^{2}} \int_{2 / Q}^{\infty} \frac{\mathrm{d} r}{r^{3}}\langle T(r)\rangle_{Y}^{2} \sim \mathrm{e}^{-Z} \int_{-\infty}^{Z} \mathrm{~d} z \mathrm{e}^{z} \operatorname{Erfc}^{2}\left(\frac{z}{\sigma}\right) .
$$

is dominated by its lower end at $r \sim 1 / Q$ (that is, by $z=Z$ ), so like the corresponding contribution (4.20) to the inclusive cross-section.

This points towards an important physical difference between the mean-field (or intermediate energy) behaviour and the behaviour at high energy, which deserves a more qualitative explanation. To that aim, notice that the measure $\mathrm{d} r / r^{3}$ in the aligned-jet integral favors the small dipoles, so the integral would be naturally dominated by its lower end at $r \sim 1 / Q$ if there was not for the strong suppression of the dipole sizes $r \ll 1 / Q_{s}$ introduced by the dipole amplitude squared $T^{2}$. Such a strong suppression occurs, as we have seen, for the mean-field amplitude $\bar{T}$, and also for the typical events in the statistical ensemble in QCD at high energy and large $Q^{2} \gg\left\langle Q_{s}^{2}\right\rangle$ : indeed, a typical event has $Q_{s} \sim\left\langle Q_{s}\right\rangle$ and thus yields a small contribution, of order $\left\langle Q_{s}^{2}\right\rangle / Q^{2}=\mathrm{e}^{-Z}$, to the integral. However, due to the front dispersion in the presence of fluctuations, the statistical ensemble contains also fronts which are at saturation at the minimal size $1 / Q$, and each such a front yields a relatively large contribution, of $O(1)$. Although such fluctuations are relatively rare, their contribution weighted by the respective probability $\sim \exp \left(-Z^{2} / \sigma^{2}\right)$ is still larger than that of the typical fronts, which behaves like $\mathrm{e}^{-Z}$. In other terms, the convolution peaks up those rare gluon configurations, or 'black spots', in the target wavefunction which are at saturation at the scale $Q^{2}$ set by the virtual photon.

Therefore, at high energy - and in contrast to what happens in the mean-field approximation, or at intermediate energies - the inclusive and diffractive cross-sections receive 
their dominant contributions from the same physical configurations, namely the small dipoles with size $r \sim 1 / Q$. In fact, a qualitative analysis similar to that in Eqs. (4.18)(4.22) reveals that the analogy between inclusive and diffractive processes at high energy is even stronger: In the large- $Q^{2}$ regime defined by Eq. (4.17), both types of cross-sections are dominated by symmetric dipole configurations within the transverse sector. In particular, the dominant behaviour of $\sigma_{\text {diff }}^{q \bar{q}}$ can be estimated as in Eq. (4.21), and reads

$$
\frac{\mathrm{d} \sigma_{\text {diff }}^{q \bar{q}}}{\mathrm{~d}^{2} b} \simeq S_{\text {diff }}^{T} \sim \frac{\sigma^{4}}{Z^{3}} \exp \left(-\frac{2 Z^{2}}{\sigma^{2}}\right) \quad \text { for } \quad \sigma \ll Z \ll \sigma^{2},
$$

which implies the following estimate for the (diffractive/inclusive) ratio $R$ :

$$
R \sim \frac{\sigma}{Z} \exp \left(-\frac{Z^{2}}{\sigma^{2}}\right) \quad \text { for } \quad \sigma \ll Z \ll \sigma^{2} .
$$

This is a scaling function, which decreases quite rapidly with $Q^{2}$ at fixed $Y$ (unlike the respective mean field estimate in Eq. (4.15)), but one should notice that this decrease becomes significant only at very large $Q^{2}$, such that $Z \gg \sigma$.

To make these estimates more precise, let us compute the dominant behaviour of $\sigma_{\text {diff }}^{q \bar{q}}$ in the regime $\sigma \gamma_{0} \gg 1$ and $Z \ll \sigma^{2}$. This is obtained by evaluating the convolution in Eq. (4.6) with the dipole amplitude $\langle T(r)\rangle$ in Eq. (3.13). Consider first the longitudinal cross section. Using Eq. (A.2) and defining $u=r Q$ and $\tau=Q /\left\langle Q_{s}\right\rangle$ we have

$$
\frac{\mathrm{d} \sigma_{\mathrm{diff}}^{L}}{\mathrm{~d}^{2} b}=\frac{\pi F}{2} \int_{0}^{1} \mathrm{~d} v \int_{0}^{\infty} \mathrm{d} u 4 v^{2}(1-v)^{2} u \mathrm{~K}_{0}^{2}(u \sqrt{v(1-v)}) \operatorname{Erfc}^{2}\left(\frac{\ln \left(\tau^{2} / u^{2}\right)}{\sigma}\right) .
$$

When $\sigma$ is large we can set $u=1$ in the argument of the error function; indeed, as previously explained, the convolution is dominated by $r \sim 1 / Q$. Then the integrals over $u$ and $v$ are easily performed and we arrive at (note that $\ln \tau^{2}=\ln \left(Q^{2} /\left\langle Q_{s}^{2}\right\rangle\right)=Z$ )

$$
\frac{\mathrm{d} \sigma_{\mathrm{diff}}^{L}}{\mathrm{~d}^{2} b}=\frac{\pi F}{6} \operatorname{Erfc}^{2}\left(\frac{Z}{\sigma}\right)
$$

Now let us look at the transverse cross section. It reads

$$
\frac{\mathrm{d} \sigma_{\mathrm{diff}}^{T}}{\mathrm{~d}^{2} b}=\frac{\pi F}{2} \int_{0}^{1} \mathrm{~d} v \int_{0}^{\infty} \mathrm{d} u v(1-v)\left[v^{2}+(1-v)^{2}\right] u \mathrm{~K}_{1}^{2}(u \sqrt{v(1-v)}) \operatorname{Erfc}^{2}\left(\frac{\ln \left(\tau^{2} / u^{2}\right)}{\sigma}\right) .
$$

The behavior of the last factor in the small- $u$ region is crucial since it cancels the logarithmic singularity of the remaining part of the integrand at $u=0$. Hence, we cannot set $u=1$ as we did for the longitudinal case. Here it is convenient to define the function

$$
\Phi_{2}(x) \equiv \int_{x}^{\infty} \mathrm{d} v \operatorname{Erfc}^{2}(v)=\frac{2}{\sqrt{\pi}} \exp \left(-x^{2}\right) \operatorname{Erfc}(x)-x \operatorname{Erfc}^{2}(x)-\sqrt{\frac{2}{\pi}} \operatorname{Erfc}(\sqrt{2} x),
$$

which has the following behavior in the various limits 


$$
\Phi_{2}(x)= \begin{cases}4|x|-\frac{2 \sqrt{2}}{\sqrt{\pi}}-\frac{\exp \left(-2 x^{2}\right)}{4 \pi|x|^{3}} & \text { for } x \ll-1 \\ \frac{2-\sqrt{2}}{\sqrt{\pi}} & \text { for } x=0 \\ \frac{\exp \left(-2 x^{2}\right)}{4 \pi x^{3}} & \text { for } x \gg 1 .\end{cases}
$$

Using Eq. (4.36) we can write the last factor in Eq. (4.35) as

$$
\operatorname{Erfc}^{2}\left(\frac{\ln \left(\tau^{2} / u^{2}\right)}{\sigma}\right)=\frac{\sigma}{2} u \frac{\mathrm{d}}{\mathrm{d} u} \Phi_{2}\left(\frac{\ln \left(\tau^{2} / u^{2}\right)}{\sigma}\right),
$$

and then the $u$-integration in Eq. (4.35) can be performed by parts. The boundary term vanishes and therefore the virtual photon transverse cross section becomes

$$
\begin{gathered}
\frac{\mathrm{d} \sigma_{\mathrm{diff}}^{T}}{\mathrm{~d}^{2} b}=\frac{\pi F}{2} \sigma \int_{0}^{1} \mathrm{~d} v \int_{0}^{\infty} \mathrm{d} u[v(1-v)]^{3 / 2}\left[v^{2}+(1-v)^{2}\right] \Phi_{2}\left(\frac{\ln \left(\tau^{2} / u^{2}\right)}{\sigma}\right) \\
\times u^{2} \mathrm{~K}_{0}(u \sqrt{v(1-v)}) \mathrm{K}_{1}(u \sqrt{v(1-v)}) .
\end{gathered}
$$

Now we can safely set $u=1$ in the argument of the $\Phi_{2}$ function since the remaining part of the integrand is well-defined for any value of $u$. The integration over $u$ and $v$ becomes straightforward and we obtain

$$
\frac{\mathrm{d} \sigma_{q \bar{q}}^{T}}{\mathrm{~d}^{2} b}=\frac{\pi F}{6} \sigma \Phi_{2}\left(\frac{Z}{\sigma}\right) .
$$

Comparing Eqs. (4.34) and (4.40) we see that the transverse cross section dominates over the longitudinal one within the whole region of interest (namely, $-\infty<Z \ll \sigma^{2}$ and $\left.\sigma \gamma_{0} \gg 1\right)$, so we finally arrive at

$$
\frac{\mathrm{d} \sigma_{\text {diff }}^{\gamma}}{\mathrm{d}^{2} b} \simeq \frac{\mathrm{d} \sigma_{\text {diff }}^{T}}{\mathrm{~d}^{2} b} \simeq \frac{\pi F}{6} \sigma \Phi_{2}\left(\frac{\ln \left(Q^{2} /\left\langle Q_{s}^{2}\right\rangle\right)}{\sigma}\right),
$$

up to corrections of relative order $Z / \sigma^{2}$ and/or $1 / \sigma$. As anticipated by the notation in Eq. (4.41), this is in fact the complete dominant contribution to the diffractive crosssection in the high-energy regime of interest (see the discussion in Sect. 4.3). This quantity has the same qualitative behaviour as the inclusive cross-section in Eq. (4.27), and this behaviour is illustrated in Figs. 15 and 16 (to be read by analogy with Figs. 13 and 14). In particular, the rescaled quantity $\left(\mathrm{d} \sigma_{\text {diff }}^{\gamma} / \mathrm{d}^{2} b\right) / \sigma$ exhibits diffusive scaling.

Finally, the ratio $R$ of the diffractive to the total cross section is easily obtained from Eqs. (4.41) and (4.27). It reads

$$
R=\frac{1}{2} \frac{\Phi_{2}\left(\ln \left(Q^{2} /\left\langle Q_{s}\right\rangle^{2}\right) / \sigma\right)}{\Phi_{1}\left(\ln \left(Q^{2} /\left\langle Q_{s}\right\rangle^{2}\right) / \sigma\right)} .
$$

For small values of $Q$ such that $\ln \left(\left\langle Q_{s}^{2}\right\rangle / Q^{2}\right) \gg \sigma$ the ratio approaches 1, it is constant and equal to $1-\sqrt{2} / 2=0.293$ in the wide interval $\left|\ln \left(Q^{2} /\left\langle Q_{s}^{2}\right\rangle\right)\right| \ll \sigma$ around the saturation 


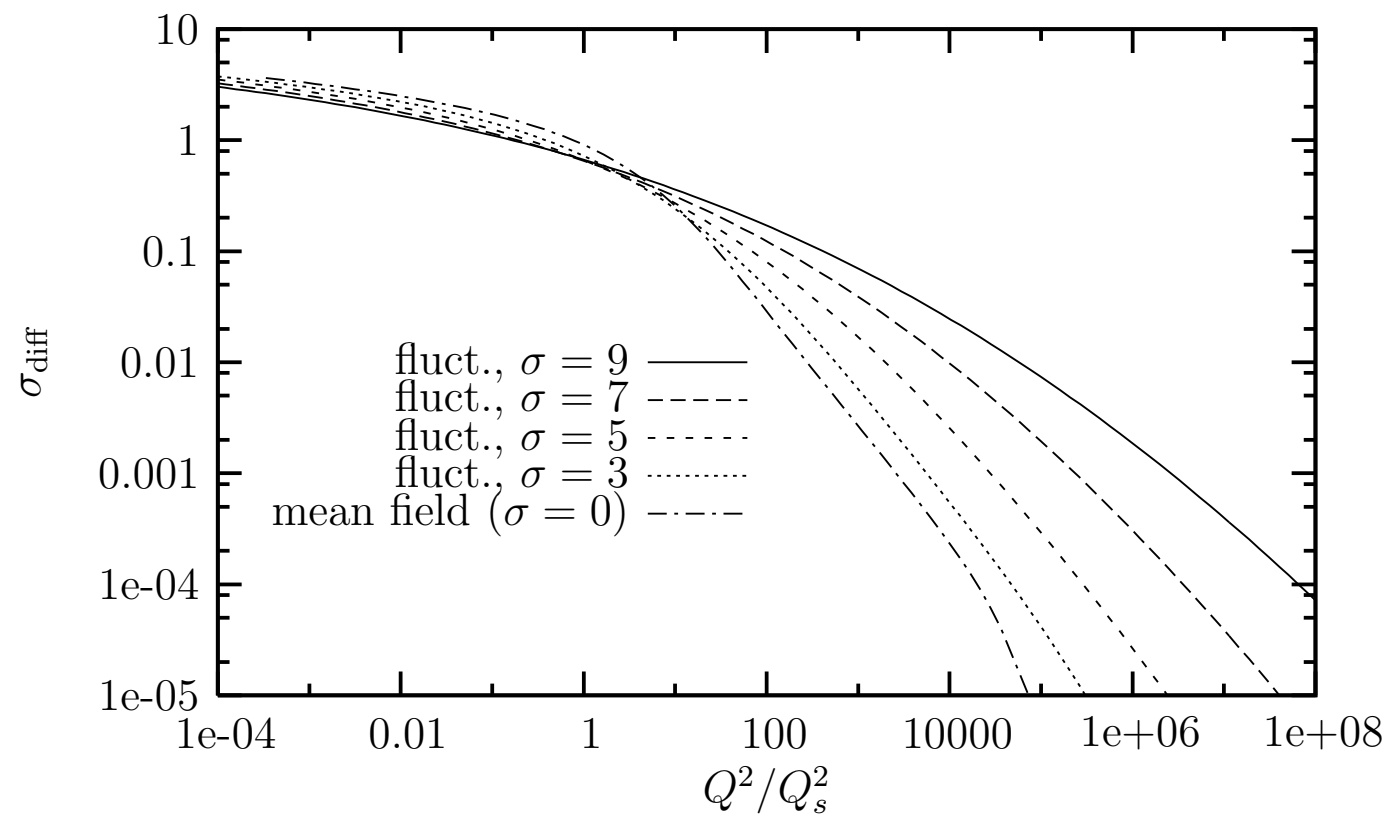

Fig. 15. The contribution of the $q \bar{q}$ component of the onium wavefunction to the diffractive cross section as a function of $Q^{2} /\left\langle Q_{s}^{2}\right\rangle$ and for various values of the front dispersion.

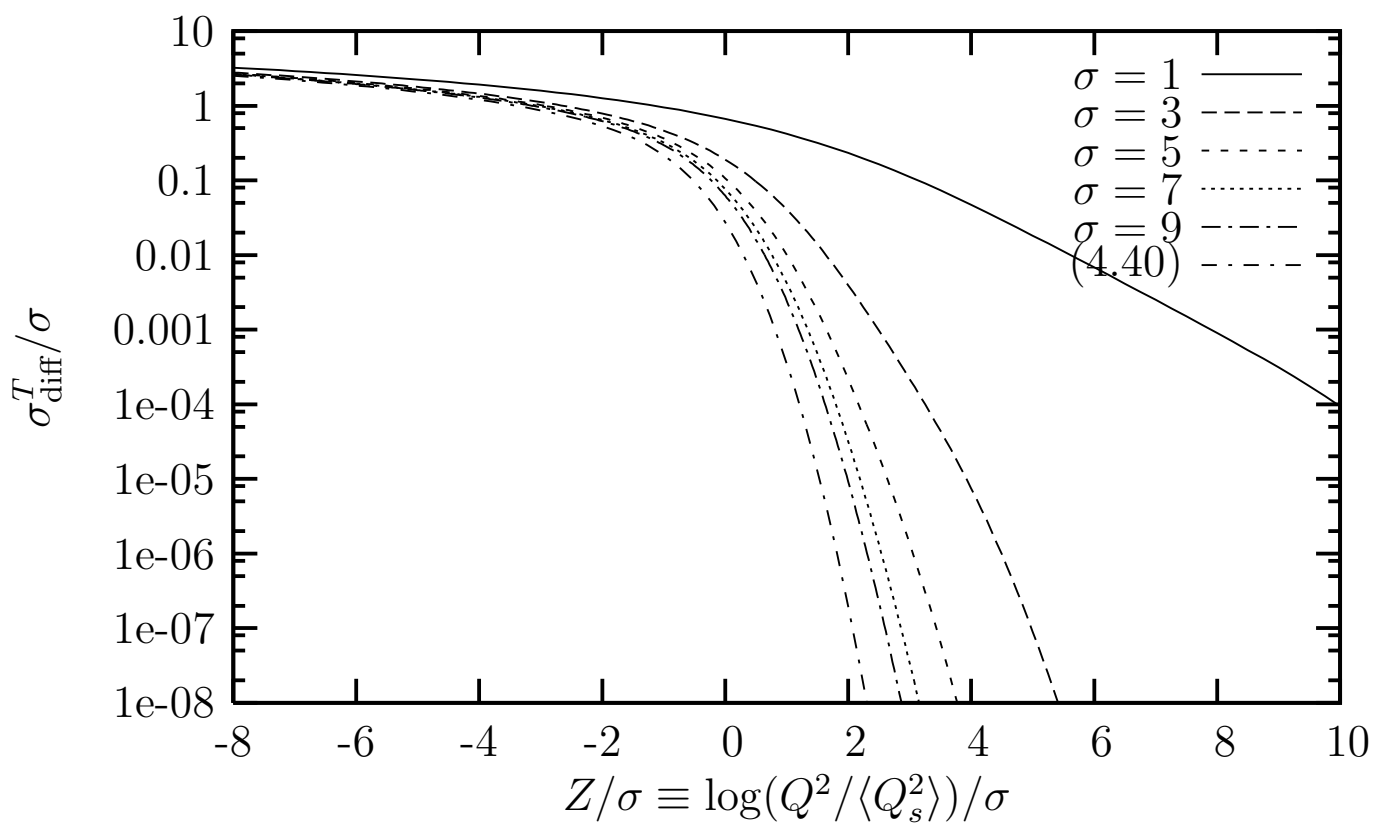

Fig. 16. The same as in Fig. 15 (divided by the front dispersion $\sigma$ ) as a function of $Z / \sigma$.

line, while it falls very fast with increasing momentum when $\ln \left(Q^{2} /\left\langle Q_{s}^{2}\right\rangle\right) \gg \sigma$ with the precise form easily inferred from Eqs. (4.37) and (4.26). This behaviour is graphically illustrated in Fig. 17, and compared to the corresponding mean-field behaviour. 


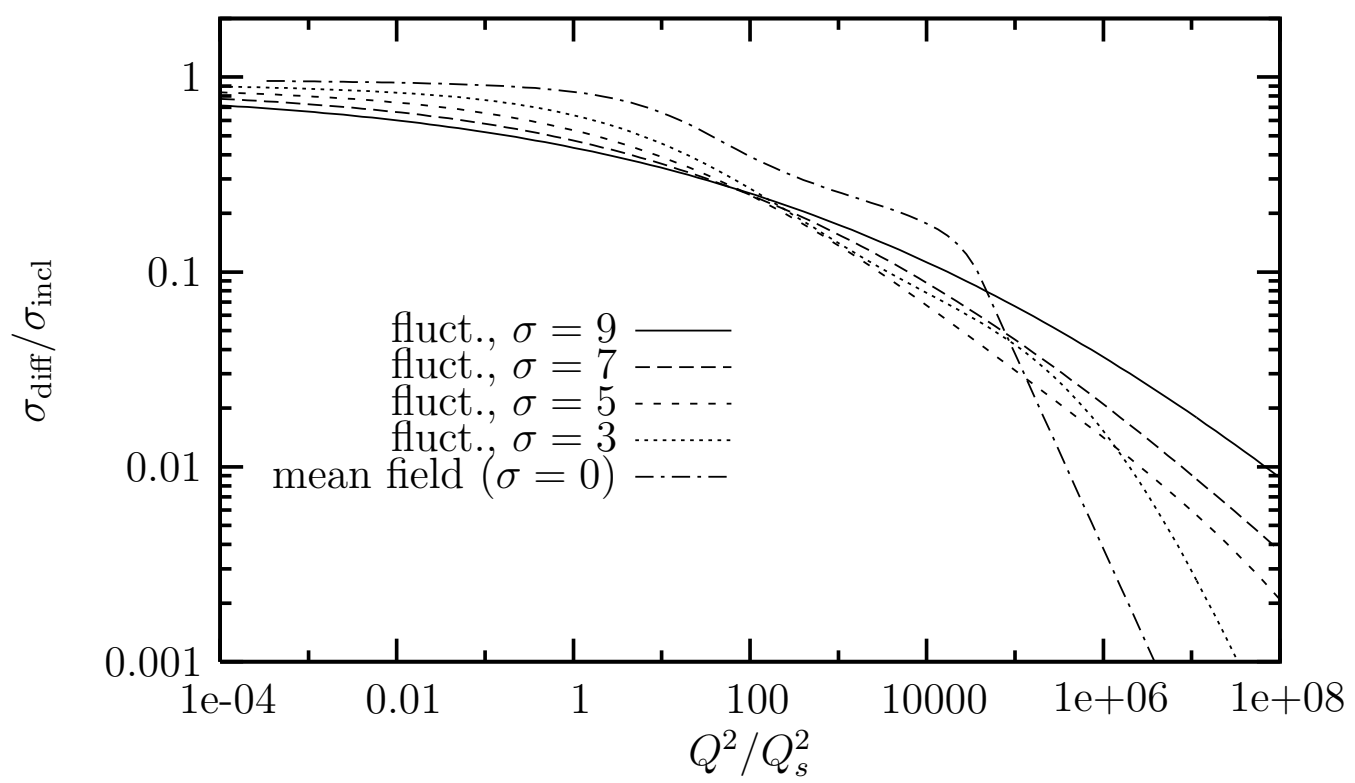

Fig. 17. The ratio of the diffractive (contribution of the $q \bar{q}$ component) to the total cross section as a function of $Q^{2} /\left\langle Q_{s}^{2}\right\rangle$.

\subsection{The $q \bar{q} g$ component}

In this subsection, we shall analyze the first non-trivial contribution to the inelastic diffraction, which arises when the wavefunction of the projectile at the time of scattering is a superposition of two Fock space components: the original $q \bar{q}$ pair (one dipole) and its $q \bar{q} g$ excitation (i.e., two dipoles at large $N_{c}$ ). This configuration is physically relevant for relatively large diffractive mass $M_{X}^{2} \gg Q^{2}$, or small values of $\beta \ll 1$, but such that $\bar{\alpha}_{s} \ln (1 / \beta) \ll 1$, in order for the effects of the higher Fock states in the projectile to be negligible. As we shall see, the two-component configuration generates contributions of order $\bar{\alpha}_{s} \ln (1 / \beta)$ to both the elastic and the inelastic pieces of the diffractive cross-section, but at high energy, the inelastic piece is parametrically suppressed with respect to the elastic one in the whole kinematical range of interest (including the large $-Q^{2}$ domain defined by Eq. (4.17), which extends with $Y$ ). This means that, if one starts at $Y=Y_{\text {gap }}$, where the diffraction is purely elastic (cf. Eq. (2.10)), and one increases $Y$ for a fixed value of $Y_{\text {gap }}$ (i.e., one increases the rapidity of the projectile), then the elastic piece of diffraction will rise faster than the inelastic one, and thus will remain the dominant contribution to $\sigma_{\text {diff }}$ at any $Y \geq Y_{\text {gap }}$ (i.e., for arbitrarily small ${ }^{12} \beta$ ). This situation occurs for sufficiently high values of $Y_{\text {gap }}$, such that $\sigma\left(Y_{\text {gap }}\right) \gg 1$, and is in sharp contrast with the corresponding mean-field scenario $[43,50]$, where for $Q^{2} \gg Q_{s}^{2}$ the inelastic diffraction rises faster than the elastic one with $1 / \beta$ and thus rapidly dominates over the latter.

One should also remind here that the diffractive cross-section that we have in mind, cf. Eqs. (2.1) and (4.2), is integrated over all the values of the rapidity gap between $Y_{\text {gap }}$ and $Y$. But, of course, the differential cross-section $\mathrm{d} \sigma_{\text {diff }} / \mathrm{d} \ln (1 / \beta)$ at sufficiently small $\beta \ll 1$

$\overline{12}$ Strictly speaking, our argument applies so long as $\bar{\alpha}_{s} \ln (1 / \beta) \ll \ln \left(1 / \alpha_{s}^{2}\right)$, i.e., for a projectile which is non-saturated, since this is the validity limit of Eq. (2.3). 
is always dominated by inelastic scattering (cf. Eq. (4.2)), because the corresponding elastic contribution is peaked near $\beta=1$. In what follows, we shall compute the lowest order contribution to this differential cross-section, as given by the $q \bar{q} g$ state.

\subsubsection{Diffractive DIS with one or two dipoles}

Within the present approximations, the scattering probabilities for an onium made with two dipoles are obtained by taking $N=2$ in the general formulæ in Sect. 2 and using the dipole probabilities $P_{1}$ and $P_{2}$ given by Eq. (2.14) with $\mathrm{d} Y=Y-Y_{0}=\ln (1 / \beta)$.

We are primarily interested in diffraction, but in order to separate its elastic and inelastic components, we also need the forward amplitude $\mathcal{A}(\boldsymbol{x}, \boldsymbol{y} ; Y)$, so let us start with this latter. According to Eq. (2.9), the forward amplitude can be expressed either as the scattering of a single $q \bar{q}$ dipole in the frame where the target has rapidity $Y$ :

$$
\mathcal{A}(\boldsymbol{x}, \boldsymbol{y} ; Y)=\langle T(\boldsymbol{x}, \boldsymbol{y})\rangle_{Y}
$$

or as the scattering of an onium composed of two dipoles, in the frame where the target has rapidity $Y_{0}$ (with the simplified notation $T_{\boldsymbol{x} \boldsymbol{y}} \equiv T(\boldsymbol{x}, \boldsymbol{y})$ ) :

$$
\begin{aligned}
\mathcal{A}(\boldsymbol{x}, \boldsymbol{y} ; Y) & =\langle T(\boldsymbol{x}, \boldsymbol{y})\rangle_{Y_{0}}+\ln (1 / \beta) \Delta \mathcal{A}\left(\boldsymbol{x}, \boldsymbol{y} ; Y_{0}\right) \\
\Delta \mathcal{A}\left(\boldsymbol{x}, \boldsymbol{y} ; Y_{0}\right) & \equiv \frac{\bar{\alpha}_{s}}{2 \pi} \int_{\boldsymbol{z}} \mathcal{M}(\boldsymbol{x}, \boldsymbol{y}, \boldsymbol{z})\left\langle T_{\boldsymbol{x} \boldsymbol{z}}+T_{\boldsymbol{z} \boldsymbol{y}}-T_{\boldsymbol{x} \boldsymbol{z}} T_{\boldsymbol{z y}}-T_{\boldsymbol{x} \boldsymbol{y}}\right\rangle_{Y_{0}}
\end{aligned}
$$

The quantity $\Delta \mathcal{A}$ describes the changes in $\mathcal{A}$ brought in by the one-step evolution, namely, the scattering of the two-dipole state (the first three terms within the brackets) together with the 'virtual' correction to the scattering of the one-dipole state (the fourth term there). By comparing Eqs. (4.43) and (4.44) and taking the limit $\mathrm{d} Y \equiv \ln (1 / \beta) \rightarrow 0$, one can immediately deduce an evolution equation for $\left\langle T_{\boldsymbol{x} \boldsymbol{y}}\right\rangle_{Y}$ :

$$
\frac{\partial\left\langle T_{\boldsymbol{x} \boldsymbol{z}}\right\rangle_{Y}}{\partial Y}=\frac{\bar{\alpha}_{s}}{2 \pi} \int_{\boldsymbol{z}} \mathcal{M}(\boldsymbol{x}, \boldsymbol{y}, \boldsymbol{z})\left\langle T_{\boldsymbol{x} \boldsymbol{z}}+T_{\boldsymbol{z} \boldsymbol{y}}-T_{\boldsymbol{x} \boldsymbol{y}}-T_{\boldsymbol{x} \boldsymbol{z}} T_{\boldsymbol{z} \boldsymbol{y}}\right\rangle_{Y},
$$

which is recognized as the first equation in both the Balitsky hierarchy [5] and in the hierarchy of equations with Pomeron loops [14]. (The differences between these two hierarchies, which express the effects of gluon number fluctuations, start with the second equation, as obeyed by the two-dipole amplitude $\left.\left\langle T\left(\boldsymbol{x}_{1}, \boldsymbol{y}_{1}\right) T\left(\boldsymbol{x}_{2}, \boldsymbol{y}_{2}\right)\right\rangle_{Y}.\right)$ Within the mean field approximation (2.32), Eq. (4.45) reduces to the BK equation (2.39).

Consider similarly the elastic probability, Eq. (2.7). This too can be evaluated in either frame (the ' $Y$-frame' and the ' $Y_{0}$-frame'), with the following results :

$$
\begin{aligned}
P_{\mathrm{el}}(\boldsymbol{x}, \boldsymbol{y} ; Y) & =\left|\langle T(\boldsymbol{x}, \boldsymbol{y})\rangle_{Y}\right|^{2} \simeq\left|\langle T(\boldsymbol{x}, \boldsymbol{y})\rangle_{Y_{0}}\right|^{2}+\ln (1 / \beta) \Delta P_{\mathrm{el}}\left(\boldsymbol{x}, \boldsymbol{y} ; Y_{0}\right) \\
\Delta P_{\mathrm{el}}\left(\boldsymbol{x}, \boldsymbol{y} ; Y_{0}\right) & \equiv 2 \operatorname{Re}\left\{\langle T(\boldsymbol{x}, \boldsymbol{y})\rangle_{Y_{0}} \Delta \mathcal{A}\left(\boldsymbol{x}, \boldsymbol{y} ; Y_{0}\right)\right\}
\end{aligned}
$$

where the expression in the $Y_{0}$-frame holds to the order of interest in $\bar{\alpha}_{s} \ln (1 / \beta)$. Clearly, $\Delta P_{\mathrm{el}}$ represents the $q \bar{q} g$ contribution to the elastic probability. 
For the diffractive probability (2.3), on the other hand, the choice of the $Y_{0}$-frame is mandatory, since $Y_{0}$ plays also the role of the physical rapidity gap. We thus find:

$$
P_{\text {diff }}\left(\boldsymbol{x}, \boldsymbol{y} ; Y, Y_{0}\right)=P_{\mathrm{diff}}^{q \bar{q}}\left(\boldsymbol{x}, \boldsymbol{y} ; Y_{0}\right)+\ln (1 / \beta) \Delta P_{\mathrm{diff}}\left(\boldsymbol{x}, \boldsymbol{y} ; Y_{0}\right),
$$

where

$$
P_{\text {diff }}^{q \bar{q}}\left(\boldsymbol{x}, \boldsymbol{y} ; Y_{0}\right) \equiv\left|\langle T(\boldsymbol{x}, \boldsymbol{y})\rangle_{Y_{0}}\right|^{2}=P_{\mathrm{el}}\left(\boldsymbol{x}, \boldsymbol{y} ; Y_{0}\right)
$$

is the contribution of the elementary $q \bar{q}$ pair, and is the same as the elastic probability corresponding to a total rapidity $Y_{0}$ (cf. Eq. (4.46)), while

$$
\begin{aligned}
\Delta P_{\mathrm{diff}}\left(\boldsymbol{x}, \boldsymbol{y} ; Y_{0}\right) & \equiv \frac{\bar{\alpha}_{s}}{2 \pi} \int_{\boldsymbol{z}} \mathcal{M}(\boldsymbol{x}, \boldsymbol{y}, \boldsymbol{z})\left\{\left|\left\langle 1-S_{\boldsymbol{x} \boldsymbol{z}} S_{\boldsymbol{z y}}\right\rangle_{Y_{0}}\right|^{2}-\left|\left\langle 1-S_{\boldsymbol{x} \boldsymbol{y}}\right\rangle_{Y_{0}}\right|^{2}\right\} \\
& =\frac{\bar{\alpha}_{s}}{2 \pi} \int_{\boldsymbol{z}} \mathcal{M}(\boldsymbol{x}, \boldsymbol{y}, \boldsymbol{z})\left\{\left|\left\langle T_{\boldsymbol{x} \boldsymbol{z}}+T_{\boldsymbol{z} \boldsymbol{y}}-T_{\boldsymbol{x} \boldsymbol{z}} T_{\boldsymbol{z} \boldsymbol{y}}\right\rangle_{Y_{0}}\right|^{2}-\left|\left\langle T_{\boldsymbol{x} \boldsymbol{y}}\right\rangle_{Y_{0}}\right|^{2}\right\}
\end{aligned}
$$

represents the additional contribution due to the $q \bar{q} g$ state. This contains both elastic and inelastic components, and in order to separate the latter we use $P_{\text {diff }}^{\text {inel }}=P_{\text {diff }}-P_{\text {el }}$, together with Eqs. (4.46) and (4.48). This yields:

$$
\begin{aligned}
P_{\text {diff }}^{\text {inel }}\left(\boldsymbol{x}, \boldsymbol{y} ; Y, Y_{0}\right) & =\ln (1 / \beta) \Delta P_{\text {inel }}\left(\boldsymbol{x}, \boldsymbol{y} ; Y_{0}\right) \\
\Delta P_{\text {inel }}\left(\boldsymbol{x}, \boldsymbol{y} ; Y_{0}\right) & \equiv \Delta P_{\text {diff }}\left(\boldsymbol{x}, \boldsymbol{y} ; Y_{0}\right)-\Delta P_{\mathrm{el}}\left(\boldsymbol{x}, \boldsymbol{y} ; Y_{0}\right) .
\end{aligned}
$$

From the expressions (4.44), (4.46) and (4.49), one immediately finds

$$
\Delta P_{\text {inel }}\left(\boldsymbol{x}, \boldsymbol{y} ; Y_{0}\right)=\frac{\bar{\alpha}_{s}}{2 \pi} \int_{\boldsymbol{z}} \mathcal{M}(\boldsymbol{x}, \boldsymbol{y}, \boldsymbol{z})\left|\left\langle T_{\boldsymbol{x} \boldsymbol{z}}+T_{\boldsymbol{z} \boldsymbol{y}}-T_{\boldsymbol{x} \boldsymbol{y}}-T_{\boldsymbol{x} \boldsymbol{z}} T_{\boldsymbol{z} \boldsymbol{y}}\right\rangle_{Y_{0}}\right|^{2}
$$

Note that the inelastic diffraction starts at order $\bar{\alpha}_{s} \ln (1 / \beta)$, i.e., it requires at least one soft gluon in the wavefunction of the projectile.

From Eqs. (4.7) and (4.50), one can deduce our lowest-order approximation for the differential diffractive cross-section, valid for $\beta \ll 1$ but such that $\bar{\alpha}_{s} \ln (1 / \beta) \ll 1$ :

$$
\frac{\mathrm{d} \sigma_{\text {diff }}^{\gamma}}{\mathrm{d}^{2} b \mathrm{~d} \ln (1 / \beta)} \simeq \int_{0}^{1} \mathrm{~d} z \int \mathrm{d}^{2} \boldsymbol{r} \sum_{\alpha=L, T}\left|\psi_{\alpha}^{\gamma}(z, r ; Q)\right|^{2} \Delta P_{\text {inel }}\left(\boldsymbol{b}, \boldsymbol{r} ; Y_{0}\right)
$$

Note that, for the simple onium configuration at hand, the inelastic diffraction is, by construction, the probability to have two dipoles in the final state. Hence, the quantity (4.52) represents also the cross-section for gluon production in diffractive DIS [45, 52], at the present level of accuracy.

\subsubsection{The inelastic component of diffraction}

The inelastic diffraction probability associated with the $q \bar{q} g$ state is given by Eq. (4.51), which after the trivial change of variable $\boldsymbol{z} \equiv \boldsymbol{x}-\boldsymbol{s}$ is rewritten as (with $\boldsymbol{r}=\boldsymbol{x}-\boldsymbol{y}$ ) : 


$$
\Delta P_{\text {inel }}(r)=\frac{\bar{\alpha}_{s}}{2 \pi} \int_{\boldsymbol{s}} \frac{\boldsymbol{r}^{2}}{\boldsymbol{s}^{2}(\boldsymbol{r}-\boldsymbol{s})^{2}}\langle T(\boldsymbol{s})+T(\boldsymbol{r}-\boldsymbol{s})-T(\boldsymbol{r})-T(\boldsymbol{s}) T(\boldsymbol{r}-\boldsymbol{s})\rangle^{2} .
$$

In line with the previous approximations, we have assumed the high-energy amplitudes to be real and ignored their impact parameter dependence. It is also implicit in our notations that the target expectation values are evaluated at a rapidity $Y_{0}$, and therefore they involve the (average) saturation momentum $\left\langle Q_{s}^{2}\right\rangle\left(Y_{0}\right)$ and the front dispersion $\sigma^{2}\left(Y_{0}\right)=D_{\text {fr }} \bar{\alpha}_{s} Y_{0}$. We shall evaluate this expression in both the mean-field approximation and the general case with fluctuations, to emphasize the difference between these two situations.

\section{i) The mean-field approximation}

In this approximation, the two-dipole amplitude is assumed to factorize $:\langle T(\boldsymbol{s}) T(\boldsymbol{r}-\boldsymbol{s})\rangle \approx$ $\bar{T}(\boldsymbol{s}) \bar{T}(\boldsymbol{r}-\boldsymbol{s})$, with $\bar{T}(\boldsymbol{r})$ the mean-field amplitude of Eq. (4.8). The most interesting situation is when the external dipole is small, $r \ll 1 / Q_{s}$, and for that case one can distinguish between three different physical regions for the internal dipoles $s$ and $\boldsymbol{r}-\boldsymbol{s}$ : (i) one of the two dipoles, say $s$, is much smaller than the external one: $s \ll r$; (ii) both internal dipoles are much larger than the external one, but they are still small as compared to the saturation length: $r \ll s \simeq|\boldsymbol{r}-s| \ll 1 / Q_{s}$; (iii) both internal dipoles are at saturation: $s \simeq|\boldsymbol{r}-\boldsymbol{s}| \gtrsim 1 / Q_{s}$. Corresponding to these three cases, the integral in Eq. (4.53) is decomposed into three pieces, which are estimated as (up to irrelevant numerical factors)

$$
\Delta P_{\text {inel }}(r) \sim \bar{\alpha}_{s}\left[\int_{0}^{r} \frac{\mathrm{d} s}{s}\left(s^{2} Q_{s}^{2}\right)^{2 \gamma}+r^{2} \int_{r}^{1 / Q_{s}} \frac{\mathrm{d} s}{s^{3}}\left(s^{2} Q_{s}^{2}\right)^{2 \gamma}+r^{2} \int_{1 / Q_{s}}^{\infty} \frac{\mathrm{d} s}{s^{3}}\right] \sim \bar{\alpha}_{s} r^{2} Q_{s}^{2}(
$$

The dominant contribution, as isolated in the r.h.s., comes from relatively large internal dipoles with sizes $s \sim 1 / Q_{s} \gg r$ (i.e., from domains (ii) and (iii) alluded to above). Note the similarity between this calculation and that of the $q \bar{q}$ contribution to the diffractive cross-section in the MFA (cf. Eq. (4.14)) : the external dipole size $r$ plays here the same role as the resolution scale $1 / Q$ in Eq. (4.14), and the dipole kernel has limiting behaviours similar to those of the transverse virtual photon wavefunction (cf. Eq. (4.9)).

The case of a relatively large dipole $r \gg 1 / Q_{s}$ is technically more involved, but the analysis in Ref. [50] shows that, in that case, $\Delta P_{\text {inel }}(r)$ vanishes very fast - at least as fast as $\bar{S}^{2}(r) \equiv(1-\bar{T}(r))^{2}$ - when increasing $r$.

To summarize, in the mean field approximation, $\Delta P_{\text {inel }}(r)$ increases with $r$ like $r^{2}$ at $r^{2} Q_{s}^{2} \ll 1$, it decreases very fast at $r^{2} Q_{s}^{2} \gg 1$, and it develops a maximum at $r \sim 1 / Q_{s}$ [50]. This behaviour is manifest on the "mean field" curve in Fig. 18.

\section{ii) The high-energy behaviour with fluctuations}

We shall now extract the dominant behaviour of the integral in Eq. (4.53) in the highenergy regime at $\sigma \gg 1 / \gamma_{0}$ and $z \equiv \ln \left(1 / r^{2}\left\langle Q_{s}^{2}\right\rangle\right) \ll \sigma^{2}$. We anticipate that, in this regime, the dominant contribution comes from internal dipoles with sizes comparable to 
that of the external one, $s \sim|\boldsymbol{r}-\boldsymbol{s}| \sim r$, hence one can use the high-energy estimates (3.13)-(3.14) for all the amplitudes which appear in Eq. (4.53).

Eq. (3.14) implies $\langle T(\boldsymbol{s}) T(\boldsymbol{r}-\boldsymbol{s})\rangle=\left\langle T\left(r_{<}\right)\right\rangle$, where $r_{<}=\min (s,|\boldsymbol{r}-\boldsymbol{s}|)$; thus, the quadratic and one of the linear terms in Eq. (4.53) cancel each other, and the integral reduces to

$$
\Delta P_{\text {inel }}=2 \frac{\bar{\alpha}_{s}}{2 \pi} \int_{\boldsymbol{s}} \frac{\boldsymbol{r}^{2}}{\boldsymbol{s}^{2}(\boldsymbol{r}-\boldsymbol{s})^{2}}[\langle T(\boldsymbol{s})\rangle-\langle T(\boldsymbol{r})\rangle]^{2}
$$

where the integration is now restricted to the half plane $|s| \geq|\boldsymbol{r}-\boldsymbol{s}|$. It is convenient to change the integration variable to $u=s / r$. Then the above equation becomes

$$
\Delta P_{\text {inel }}=4 \frac{\bar{\alpha}_{s}}{2 \pi} \int_{1 / 2}^{\infty} \frac{\mathrm{d} u}{u} \int_{0}^{\phi_{0}} \frac{\mathrm{d} \phi}{1+u^{2}-2 u \cos \phi}[\langle T(u r)\rangle-\langle T(r)\rangle]^{2},
$$

where the upper limit is $\phi_{0}=\arccos (1 / 2 u)$. Since the scattering amplitudes in Eq. (4.56) do not depend on the angle $\phi$, one can perform the angular integration to obtain

$$
\Delta P_{\text {inel }}=8 \frac{\bar{\alpha}_{s}}{2 \pi} \int_{1 / 2}^{\infty} \mathrm{d} u \frac{\arctan \left(\frac{(1+u)}{|1-u|} \sqrt{\frac{2 u-1}{2 u+1}}\right)}{u(1+u)|1-u|}[\langle T(u r)\rangle-\langle T(r)\rangle]^{2} .
$$

One can check that the above integrand vanishes as $1 / u^{3}$ for large $u$. Thus, the dominant contribution comes from $u=O(1)$, that is, from internal dipoles sizes $s$ such that $s \sim r$, as anticipated. By using this property together with the specific form of the amplitude given in Eq. (3.13), we can expand

$$
\langle T(u r)\rangle-\langle T(r)\rangle \simeq-\frac{\ln u^{2}}{\sigma} \frac{\partial\langle T\rangle}{-\partial\left(\ln \left(r^{2}\left\langle Q_{s}^{2}\right\rangle\right) / \sigma\right)}=\frac{\ln u^{2}}{\sqrt{\pi} \sigma} \exp \left(-\frac{\ln ^{2}\left(r^{2}\left\langle Q_{s}^{2}\right\rangle\right)}{\sigma^{2}}\right),
$$

where we have kept only the first term in the Taylor expansion, since the other terms are suppressed by higher powers of $\ln \left(u^{2}\right) / \sigma$ and thus are truly negligible at high energy. After the expansion (4.58), the result of the integration is a pure number, so we arrive at

$$
\Delta P_{\text {inel }}(r) \simeq \frac{\bar{\alpha}_{s}}{2 \pi} \frac{J}{\pi \sigma^{2}} \exp \left(-\frac{2 z^{2}}{\sigma^{2}}\right) \quad \text { for } \quad z \ll \sigma^{2},
$$

with $J$ numerically computed as $J=15.1$.

The above result is valid for both small and large dipoles and shows that $\Delta P_{\text {inel }}(r)$ is an even function of $z \equiv \ln \left(1 / r^{2}\left\langle Q_{s}^{2}\right\rangle\right)$, which rapidly vanishes when $|z| \gg \sigma$ and has a maximum at $r^{2}\left\langle Q_{s}^{2}\right\rangle=1$. This behaviour is compared to the corresponding mean field behaviour in Fig. 18 which shows that, when increasing the energy, the maximum around $r^{2} \sim 1 /\left\langle Q_{s}^{2}\right\rangle$ becomes flatter and flatter. The emergence of the diffusive scaling with increasing $\sigma$ and the convergence towards the asymptotic form (4.59) are illustrated in Fig. 19. (The various curves in Figs. 18 and 19 are obtained by numerically evaluating the r.h.s. of Eq. (4.53) with the dipole amplitude in Eq. (3.17) with $\gamma=1$, together with a corresponding expression for the two-dipole amplitude $\langle T(\boldsymbol{s}) T(\boldsymbol{r}-\boldsymbol{s})\rangle$.) 


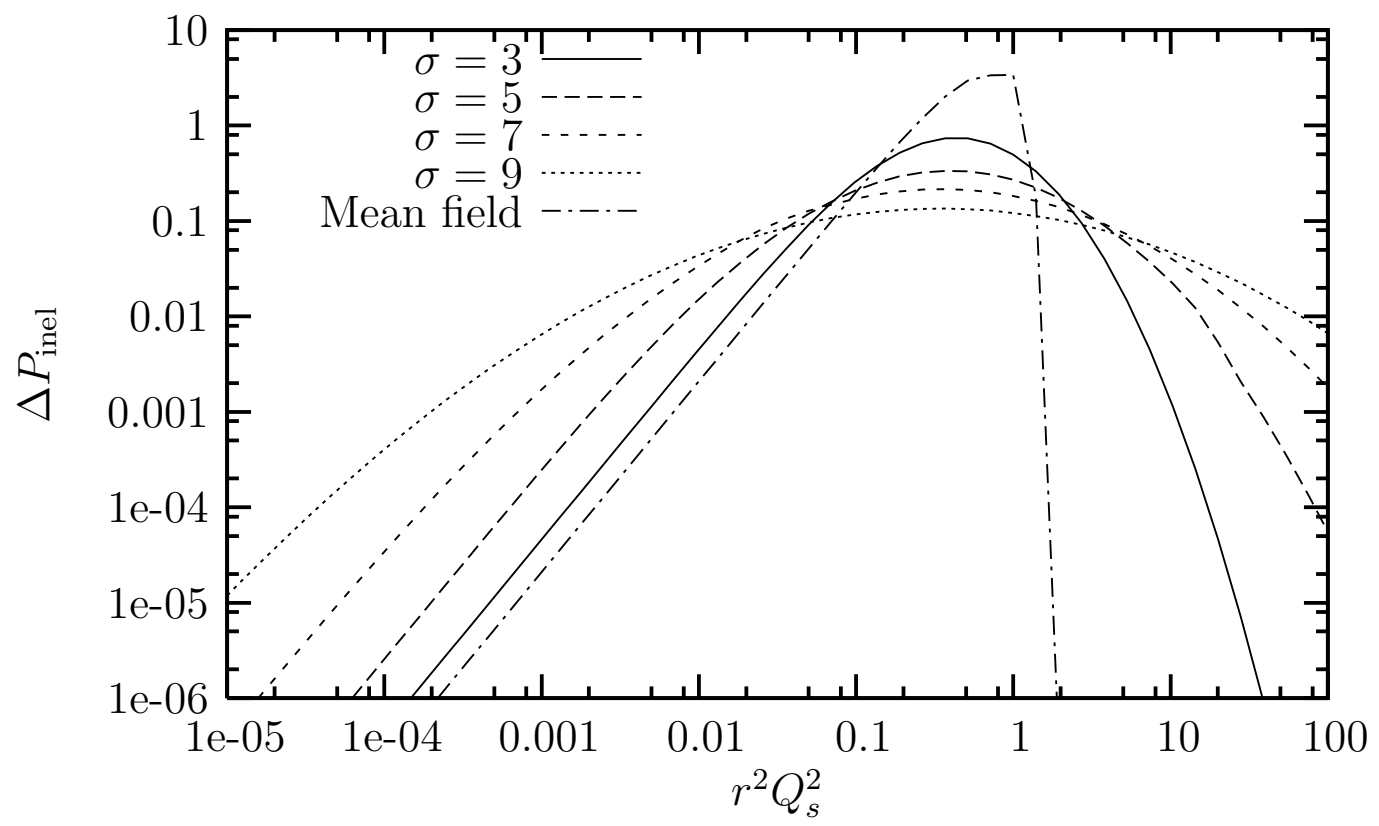

Fig. 18. The contribution of the $q \bar{q} g$ component of the onium wavefunction to the inelastic diffraction probability as a function of $r^{2}\left\langle Q_{s}^{2}\right\rangle$ and for various values of the front dispersion.

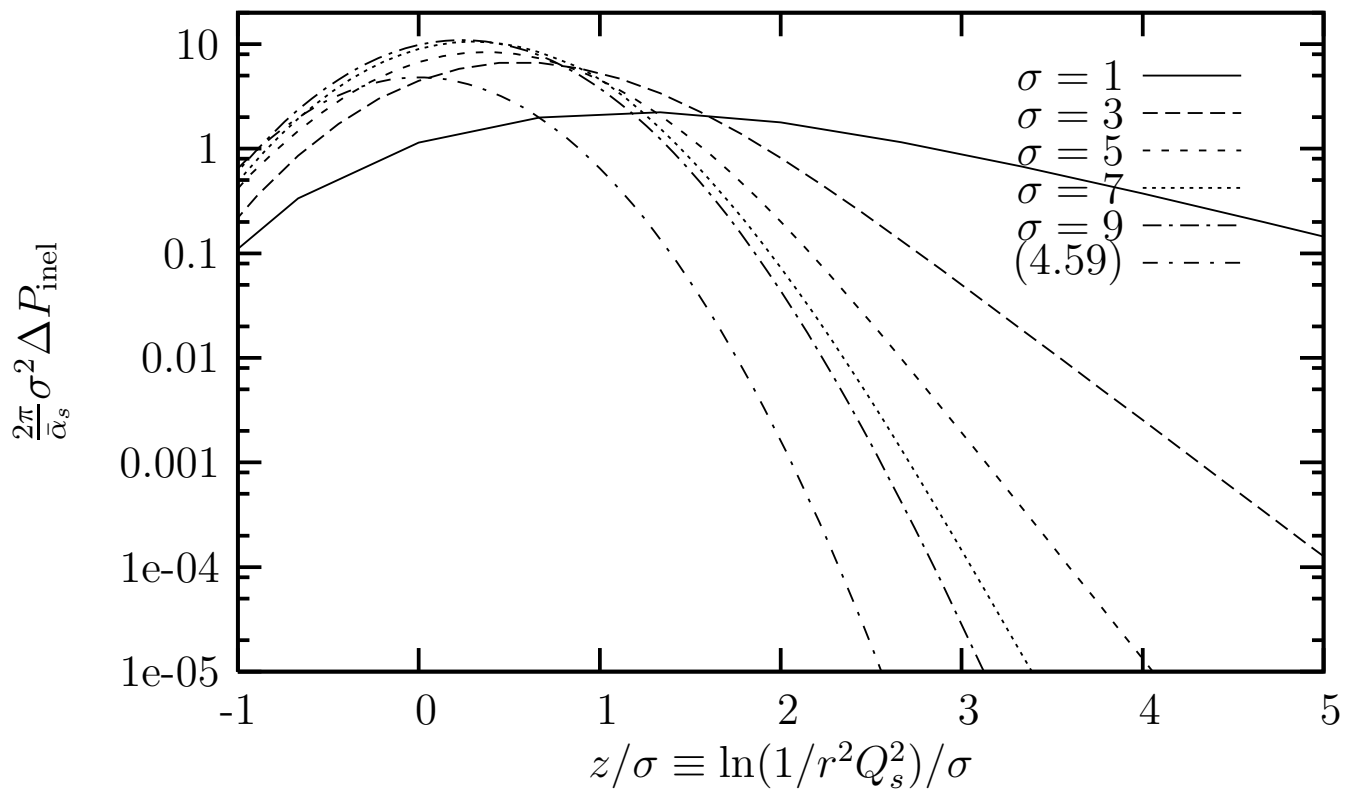

Fig. 19. The same as in Fig. 18 (multiplied by $\sim \sigma^{2}$ ) as a function of $z / \sigma$.

By inserting the above result (4.59) into the r.h.s. of Eq. (4.52) and performing the convolutions with the photon wavefunctions, we can compute the lowest-order contribution to the differential diffractive cross-section at high energy and $\beta \ll 1$. (As previously explained, this quantity is the same as the the cross-section for gluon production in diffractive DIS.) Via manipulations similar to those encountered before (cf. Sect. 4.2.2), one finds 


$$
\frac{\mathrm{d} \sigma_{\mathrm{diff}}^{L}}{\mathrm{~d}^{2} b \mathrm{~d} \ln (1 / \beta)} \simeq \frac{\bar{\alpha}_{s}}{2 \pi} \frac{2 J F}{3} \frac{1}{\sigma^{2}} \exp \left(-\frac{2 Z^{2}}{\sigma^{2}}\right)
$$

for the longitudinal piece and, respectively,

$$
\frac{\mathrm{d} \sigma_{\mathrm{diff}}^{T}}{\mathrm{~d}^{2} b \mathrm{~d} \ln (1 / \beta)} \simeq \frac{\bar{\alpha}_{s}}{2 \pi} \frac{\sqrt{2 \pi} J F}{6} \frac{1}{\sigma} \operatorname{Erfc}\left(\frac{\sqrt{2} Z}{\sigma}\right)
$$

for the transverse one. As usual, $F \equiv\left(N_{c} \alpha_{\mathrm{em}} / 2 \pi^{2}\right) \sum_{f} e_{f}^{2}$, and the above results are valid in the high energy regime at $\sigma \gamma_{0} \gg 1$ and $-\infty<Z \ll \sigma^{2}$, up to corrections of $O\left(Z / \sigma^{2}\right)$.

Comparing Eqs. (4.60) and (4.61) we see that the transverse cross section dominates the longitudinal one everywhere in the region of interest. Therefore,

$$
\frac{\mathrm{d} \sigma_{\mathrm{diff}}^{\gamma}}{\mathrm{d}^{2} b \mathrm{~d} \ln (1 / \beta)} \simeq \frac{\bar{\alpha}_{s}}{2 \pi} \frac{\sqrt{2 \pi} J F}{6} \frac{1}{\sigma} \operatorname{Erfc}\left(\frac{\sqrt{2} \ln \left(Q^{2} /\left\langle Q_{s}^{2}\right\rangle\right)}{\sigma}\right) \quad \text { for } \quad \beta \ll 1 .
$$

Recall that the average saturation scale $\left\langle Q_{s}^{2}\right\rangle$ and the front dispersion $\sigma^{2}$ which enter this equation is to be evaluated at a rapidity $Y_{0}=Y_{\text {gap }}$. The cross-section (4.62) is a monotonically decreasing function of $Q^{2}$ (for fixed $Y$, that is, fixed $\sigma$ and $\left\langle Q_{s}^{2}\right\rangle$ ). Note that after multiplication by $\sigma$, this cross-section exhibits diffusive scaling.

It might be interesting to compare this high-energy estimate to the corresponding result at intermediate energies, as given by the mean field approximation (say, by the solution to the BK equation). In the high- $Q^{2}$ regime at $Q^{2} \gg Q_{s}^{2}$, the latter can be estimated by using $\Delta P_{\text {inel }} \sim \bar{\alpha}_{s} r^{2} Q_{s}^{2}$, cf. Eq. (4.54), which yields [50]

$$
\frac{\mathrm{d} \sigma_{\mathrm{diff}}^{\gamma}}{\mathrm{d}^{2} b \mathrm{~d} \ln (1 / \beta)} \sim \bar{\alpha}_{s} F \frac{Q_{s}^{2}}{Q^{2}} \ln \frac{Q^{2}}{Q_{s}^{2}} \quad \text { for } \quad Q^{2} \gg Q_{s}^{2} .
$$

This mean-field result receives contributions from dipole sizes $r$ uniformly distributed within the range $1 / Q<r<1 / Q_{s}$. By contrast, the high-energy estimate (4.62) is dominated by $r \sim 1 / Q$.

\subsubsection{The elastic diffraction}

In this subsection, we shall evaluate the increase $\Delta P_{\mathrm{el}}$ in the probability for elastic scattering due to the emission of one soft gluon (cf. Eq. (4.46)). Our purpose is to demonstrate that, in the fluctuation-dominated regime at high energy and relatively large $Q^{2}$, cf. Eq. (4.17), this increase is parametrically larger than the corresponding evolution $\Delta P_{\text {inel }}$ in the probability for inelastic diffraction, that we have already computed.

Let us start by briefly discussing the case of the mean field approximation, where the opposite situation holds: for $Q^{2} \gg Q_{s}^{2}$ (or relatively small dipole sizes), the inelastic piece dominates over the elastic one. Indeed, by using $\Delta P_{\text {el }}(r) \equiv 2\langle T(r)\rangle \Delta \mathcal{A}(r)$ together with $\Delta \mathcal{A}=\partial\langle T\rangle / \partial Y$, cf. Eqs. (4.44)-(4.45), and the expression (4.8) for the mean-field amplitude, one immediately finds: $\Delta P_{\mathrm{el}} \sim \bar{\alpha}_{s}\left(r^{2} Q_{s}^{2}\right)^{2 \gamma}$ for $r \ll 1 / Q_{s}$. This is a 'highertwist' effect, much smaller than the corresponding inelastic contribution in Eq. (4.54). 
But at high energy, $\Delta P_{\text {el }}$ turns out to be the dominant contribution, down to very small dipole sizes, namely, so long as $z \ll \sigma^{2}$. Moreover, its calculation turns out to be quite subtle, as we explain now. On one hand, it is straightforward to use $\Delta \mathcal{A}=\partial\langle T(r)\rangle / \partial Y$ together with Eq. (3.26), to deduce :

$$
\Delta P_{\mathrm{el}}(r) \simeq \lambda \bar{\alpha}_{s} \operatorname{Erfc}\left(\frac{z}{\sigma}\right) \frac{1}{\sqrt{\pi} \sigma} \exp \left(-\frac{z^{2}}{\sigma^{2}}\right),
$$

which is indeed parametrically larger (for any $z$ ) than the corresponding inelastic piece, Eq. (4.59). For instance, for small dipole sizes, or large $z \gg \sigma$, this elastic component behaves like

$$
\Delta P_{\mathrm{el}}(r) \simeq \frac{\bar{\alpha}_{s}}{\pi} \frac{\lambda}{z} \exp \left(-\frac{2 z^{2}}{\sigma^{2}}\right) \quad \text { for } \quad \sigma \ll z \ll \sigma^{2},
$$

and dominates over the inelastic piece by a large factor $\lambda \sigma^{2} / z \gg 1$, as anticipated.

On the other hand, the above estimate for $\Delta P_{\text {el }}$ has the drawback to involve the average front velocity $\lambda$, which is generally unknown. It is therefore tempting to try and compute $\Delta \mathcal{A}(r)$ directly from its integral representation (4.44) together with Eqs. (3.13)-(3.14) for the dipole amplitudes. A priori, this calculation is independent of $\lambda$ and should even enable us to determine $\lambda$, by comparison with the expected result in Eq. (3.26). But this expectation appears to be naive: the coefficient of the would-be dominant term at large $Y$ - that term precisely which should be identified as $\lambda / \sigma$ - turns out to be zero. (This can be checked via manipulations similar to those in Eqs. (4.56)-(4.58): without taking the square of the amplitudes inside the integrand, so like in Eq. (4.57), the integral vanishes exactly after the expansion (4.58).) This seems to imply the unphysical result $\lambda=0$, but in reality it only means that the approximations (3.13)-(3.14) - although the correct leading-order estimates for the average amplitudes at high energy - cannot be used to also evaluate the right hand sides of the evolution equations. The latter are sensitive to the subleading terms, which are not under control in the present approximations.

To summarize, in the high-energy regime of interest, the diffractive probability associated with the $q \bar{q} g$ component is controlled by its elastic piece, $\Delta P_{\text {diff }} \approx \Delta P_{\mathrm{el}}$, and has the dominant behaviour displayed in Eq. (4.64). Although not as symmetric as the inelastic contribution (4.59), the function (4.64) has still the properties to vanish for both very small and very large dipole sizes, and to develop a rather flat maximum at $r \sim 1 /\left\langle Q_{s}\right\rangle$. This behaviour is illustrated in Fig. 20, which also shows that, when increasing $\sigma$, the elastic piece rises faster than the inelastic one (compare to Fig. 18) and rapidly dominates over the latter at all dipole sizes, in agreement with our previous estimates.

After replacing $\Delta P_{\text {diff }} \approx \Delta P_{\text {el }}$ in Eq. (4.47) and also using Eq. (4.48), it becomes clear that the diffractive probability coincides with the elastic probability, Eq. (4.46), which demonstrates Eq. (4.4) to the order of interest in $\bar{\alpha}_{s} \ln (1 / \beta)$. Although we have considered here only the simplest evolution of the projectile (namely, the formation of the $q \bar{q} g$ state), we expect the property (4.4) to hold also for smaller values of $\beta$ (when higher Fock-space components come into play), for the reasons explained after Eq. (4.4). Assuming this to be the case, we conclude that, within the high-energy regime at $\sigma \gamma_{0} \gg 1$ and $Z \ll \sigma^{2}$, the 


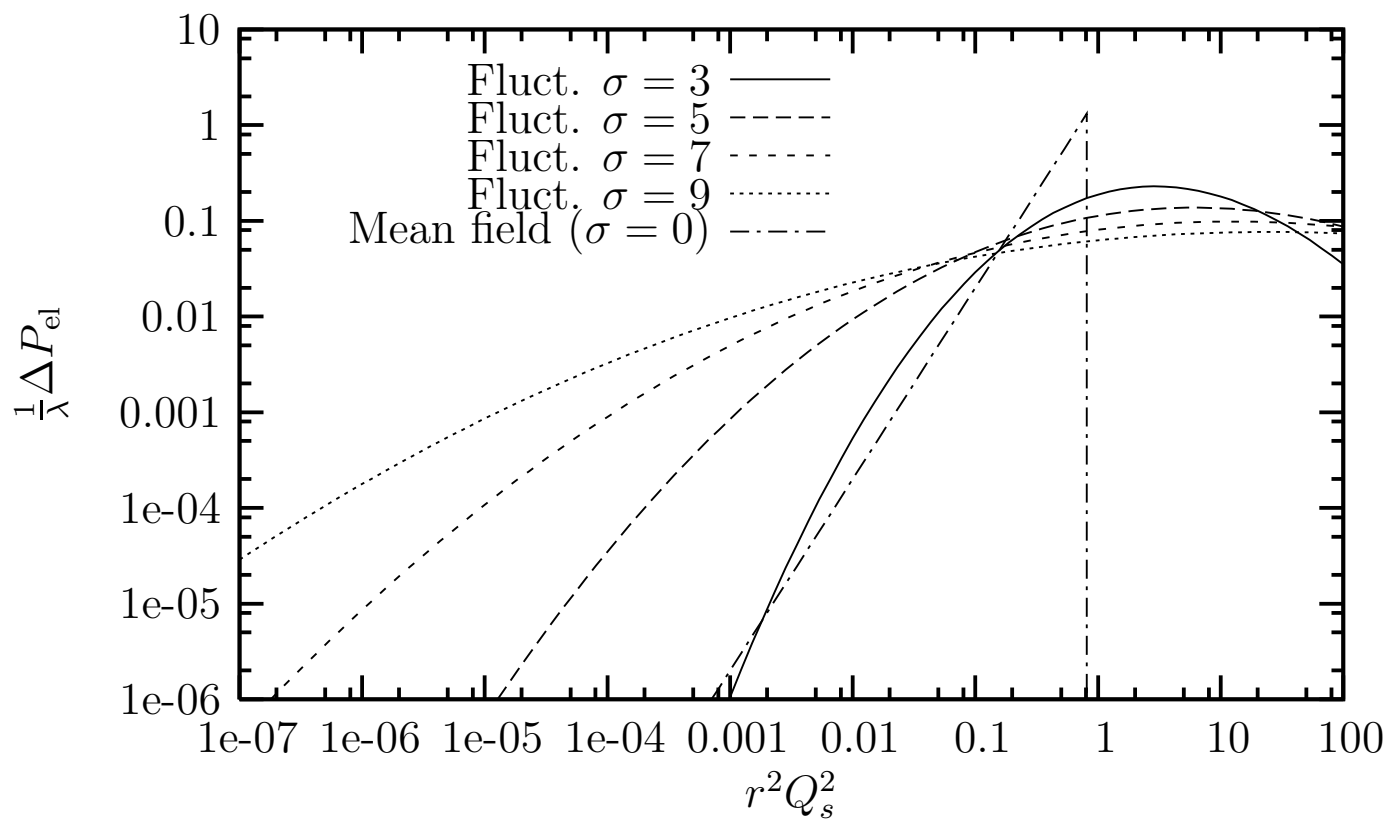

Fig. 20. The contribution of the $q \bar{q} g$ component of the onium wavefunction to the elastic diffraction probability as a function of $r^{2}\left\langle Q_{s}^{2}\right\rangle$. The high-energy estimate (4.64) is shown for various values of $\sigma$ and compared to the mean-field prediction obtained with Eq. (4.8).

diffractive DIS cross-section is dominated by the elastic scattering of the onium, and thus is independent of $\beta$ (for a given value of $Y$ ), within the limits of the leading-logarithmic approximation with respect to $\ln (1 / \beta)$. To that accuracy, the corresponding cross-section has already been evaluated in Eq. (4.41).

Let us conclude with a remark which applies to both the inclusive and the diffractive cross-sections and refers to their behaviour when increasing $Y$ in the high-energy regime: At the end of Sect. 3, we have mentioned the 'rigidity' of the dipole amplitudes at highenergy, namely, their property to increase only slowly when increasing $Y$ at fixed, but large $Q^{2}$, in the weak scattering regime. Not surprisingly, the same 'rigidity' transmits to the DIS cross-section in the diffusive-scaling window defined by Eq. (4.17). For instance, the $Y$-derivative of the diffractive cross-section in Eq. (4.41) is estimated as

$$
\frac{\mathrm{d} \sigma_{\mathrm{diff}}^{\gamma}}{\mathrm{d}^{2} b \mathrm{~d} Y} \simeq \lambda \bar{\alpha}_{s} \frac{\pi F}{6} \operatorname{Erfc}^{2}\left(\frac{\ln \left(Q^{2} /\left\langle Q_{s}^{2}\right\rangle\right)}{\sigma}\right)
$$

which for $Q^{2}$ within the range indicated in Eq. (4.17) behaves like

$$
\frac{\mathrm{d} \sigma_{\text {diff }}^{\gamma}}{\mathrm{d}^{2} b \mathrm{~d} Y} \sim \lambda \bar{\alpha}_{s} \frac{\sigma^{2}}{Z^{2}} \exp \left(-\frac{2 Z^{2}}{\sigma^{2}}\right) \sim \lambda \bar{\alpha}_{s} \frac{Z}{\sigma^{2}} \frac{\mathrm{d} \sigma_{\text {diff }}^{\gamma}}{\mathrm{d}^{2} b} \quad \text { when } \quad \sigma \ll Z \ll \sigma^{2}
$$

that is, it involves an additional suppression factor $Z / \sigma^{2} \ll 1$ as compared to the usual behaviour expected in the context of the BFKL evolution. 


\section{Conclusions}

In this paper, we have formulated the inclusive and diffractive deep inelastic scattering of a virtual photon off a hadronic target in the high-energy limit and in the large $-N_{c}$ approximation. We have worked in a Lorentz frame in which the high-energy limit is achieved by boosting the hadronic target, which then evolves to very high gluon density, whereas the rapidity of the projectile (the virtual photon) remains finite and relatively small. Our main emphasis has been on a novel type of universal behaviour which emerges at sufficiently high energy as a consequence of saturation and gluon-number fluctuations in the target wavefunction. The essential feature of this new regime is the fact that the DIS cross-sections at fixed impact parameter are dominated by the physics of saturation up to very large values of $Q^{2}$, well above the (average) saturation momentum $\left\langle Q_{s}^{2}\right\rangle$ of the target (which itself increases exponentially with $Y$ ). And the distinguished signature of this behaviour is a new, diffusive, scaling law, which should be obeyed by all the DIS cross-sections at sufficiently high energies, and which reflects the Gaussian nature of the probability distribution for the logarithm of the saturation momentum in the target.

To establish this behaviour, we have relied on two main types of ingredients: (i) a set of factorization formulæ which relate the DIS cross-sections to dipole-target scattering amplitudes, and (ii) the high-energy estimates for the latter, as determined by the Pomeron loop equations via the correspondence with statistical physics.

Concerning point (i), let us recall here that we have restricted ourselves to diffractive processes in which the hadron undergoes elastic scattering, and to leading-logarithmic approximations for the high-energy evolutions of both the target and the projectile. Besides, we have assumed the projectile to remain dilute, and thus to obey BFKL evolution. Under these assumptions, we have shown that a physically transparent description of the diffractive processes, à la Good and Walker, emerges when viewing the collision in the frame in which the rapidity of the target is equal to the minimal rapidity gap. In this frame, the final state interactions automatically cancel out, and the projectile can be represented as a distribution of dipoles which elastically scatter off the target. The effect of the collision is to destroy the coherence of the dipole superposition and thus transform the incoming photon into a collection of partons in the final state.

Concerning point (ii), we should emphasize that the asymptotic functional form of the dipole amplitudes at high energy follows from very general and elementary considerations - the average amplitudes are dominated by saturated gluon configurations, for which the single-event amplitudes have reached their unitarity limit $T=1-$, and as such it should be very robust: It is likely that this dominant behaviour will not be modified by higher order perturbative corrections, nor by corrections in $1 / N_{c}$ (but this remains to be investigated). On the other hand, the energy dependencies of the average saturation momentum $\left\langle Q_{s}^{2}\right\rangle$ and of the front dispersion $\sigma^{2}$ are sensitive to the details of the evolution, and are presently unknown for realistic values of $\alpha_{s}$. This is why, in this paper, we have systematically measured $Q^{2}$ in terms of $\left\langle Q_{s}^{2}\right\rangle$, and $Y$ in terms of $\sigma^{2}$.

Perhaps the most interesting, qualitative, conclusion of our analysis is the fact that, at sufficiently high energies and as a result of fluctuations, the physics of gluon saturation 
starts to be determinant at momenta $Q^{2}$ well above the saturation momentum $\left\langle Q_{s}^{2}\right\rangle$, within a logarithmic distance $\ln \left(Q^{2} /\left\langle Q_{s}^{2}\right\rangle\right) \sim \sigma^{2}$ which increases linearly with $Y$. From previous studies of the mean field approximation, we already knew that saturation can manifest itself at momenta larger than $Q_{s}$, via the phenomenon of geometric scaling. In the present analysis, we have shown that this phenomenon is preserved by fluctuations so long as the front dispersion is not too large (i.e., for $\sigma^{2} \ll 1$ ). What is however striking about the high-energy regime at $\sigma^{2}>1$ is that the physics above $\left\langle Q_{s}^{2}\right\rangle$ is not only influenced, but actually dominated by rare configurations at saturation. This has tremendous physical consequences, for both the dipole amplitudes and the DIS cross-sections :

- The strong correlation property $\left\langle T^{2}(r)\right\rangle \simeq\langle T(r)\rangle$, which is natural at saturation where $\langle T\rangle=1$, appears to be satisfied up to very large $Q^{2} \equiv 1 / r^{2}$, where $\langle T\rangle \ll 1$.

- The amplitudes and the DIS cross-sections are very rigid, in the sense that they rise unusually slowly when increasing $Y$ at fixed $Q^{2}$ within the weak scattering regime.

- At relatively large virtualities $Q^{2} \gg\left\langle Q_{s}^{2}\right\rangle$, cf. Eq. (4.17), the convolutions with the photon wavefunction yielding the DIS cross-sections are dominated by small sizes $r \sim$ $1 / Q$ for the $q \bar{q}$ pair, as they select those gluon configurations in the target wavefunction which are at saturation on the resolution scale set by the virtual photon.

- Similarly, the convolutions with the dipole probabilities yielding the average over the BFKL (onium) wavefunction of the projectile are dominated by dipole sizes of the order of their parent dipole - the original $q \bar{q}$ pair — and hence of order $1 / Q$.

- In the high-energy limit, the DIS diffractive cross-section becomes purely elastic (at the level of the $q \bar{q}$ pair), the inelastic contribution being parametrically suppressed.

- Within the high-energy regime defined by Eq. (3.11), all the amplitudes or crosssections show diffusive scaling, that it, they depend upon $Q^{2}$ and $Y$ only via the variable $z / \sigma$ with $z \equiv \ln \left(Q^{2} /\left\langle Q_{s}^{2}\right\rangle\right)$

- Within the diffusive scaling region alluded to above, the 'twist expansion' does not apply anymore: At large $Q^{2} \gg\left\langle Q_{s}^{2}\right\rangle$, the cross-sections decay as a Gaussian of $z / \sigma$, and not as a power of $1 / Q^{2}$.

These properties are in sharp contrast with the corresponding predictions of the MFA (that we have also worked out in this paper, at least qualitatively, for the sake of comparison), and demonstrate the breakdown of the latter at sufficiently high energy.

Note that the above conclusions depend upon the functional form of the dipole amplitudes, but not upon the precise values of coefficients $\lambda$ and $D_{\mathrm{fr}}$. But, of course, the latter are necessary in order to translate our results into physical units for $Y$ and $Q^{2}$. The small- $x$ phenomenology at HERA suggests $\lambda \simeq 0.2 \div 0.3[40,49]$, but we refrain ourselves from suggesting a similar, experimental, measure of the diffusion coefficient $D_{\mathrm{fr}}$, as we do not believe that the high-energy regime that we discuss here has been approached at HERA. (For instance, the diffractive data at HERA indicate the dominance of inelastic diffraction at small $\beta$ [50], at variance with the high-energy behaviour that we predict here.) The conceptually proper way to compute $\lambda$ and $D_{\text {fr }}$ for realistic values of $\alpha_{s}$ (and within the present approximations: high energy, leading-order perturbative QCD, and large $N_{c}$ ) would be by solving the Pomeron loop equations of Ref. [14]. This would also allow one to explicitly test our present, asymptotic, predictions, and to study the preasymptotic behaviour, like the dependence upon the initial conditions or upon the impact parameter. 
Such more detailed calculations should allow one to determine the physical region in which this high-energy behaviour should start to manifest itself and, especially, whether this region might be accessible at the LHC.

\section{Acknowledgments}

We are grateful to A. Mueller and R. Peschanski for discussions and for useful comments. Y. H. and D. T. thank SPhT Saclay for hospitality. This research was supported in part by RIKEN BNL Research Center and the U. S. Department of Energy [Contract No. DE-AC02-98CH10886]. G.S. is funded by the National Funds for Scientific Research (Belgium).

\section{A The virtual photon wavefunction}

In the dipole frame we have adopted throughout this paper, the DIS cross-sections are factorized as the product of the photon wavefunction $\left(\gamma^{*} \rightarrow q \bar{q}\right)$ with the quark-antiquark interaction amplitude. In this appendix, we give the $q \bar{q}$ dissociation probabilities for the virtual photon. This well-known process can be computed in perturbative QED (to lowest order in $\alpha_{\mathrm{em}}$ ) and reads

$$
\begin{aligned}
& \left|\psi_{T}^{\gamma}(v, r ; Q)\right|^{2}=\frac{\alpha_{\mathrm{em}} N_{c}}{2 \pi^{2}} \sum_{f} e_{f}^{2}\left\{\left(v^{2}+(1-v)^{2}\right) \bar{Q}_{f}^{2} \mathrm{~K}_{1}^{2}\left(\bar{Q}_{f} r\right)+m_{f}^{2} \mathrm{~K}_{0}^{2}\left(\bar{Q}_{f} r\right)\right\} \\
& \left|\psi_{L}^{\gamma}(v, r ; Q)\right|^{2}=\frac{\alpha_{\mathrm{em}} N_{c}}{2 \pi^{2}} \sum_{f} e_{f}^{2}\left\{4 Q^{2} v^{2}(1-v)^{2} \mathrm{~K}_{0}^{2}\left(\bar{Q}_{f} r\right)\right\}
\end{aligned}
$$

where $\bar{Q}_{f}^{2} \equiv v(1-v) Q^{2}+m_{f}^{2}, m_{f}$ and $e_{f}$ is the mass and the electric charge of the quark with flavor $f$, and $\mathrm{K}_{0}$ and $\mathrm{K}_{1}$ are the modified Bessel functions.

In order to get estimates of the DIS cross-sections, i.e. to estimate the convolution of this wavefunction with the $q \bar{q}$ interaction amplitude, we shall ignore the quark masses (these are important only for relatively small $Q^{2} \sim m_{f}^{2}$ ) and use simple approximations for the Bessel functions, as quite common in the literature (see, e.g., [40] for similar manipulations). Specifically, since $\mathrm{K}_{\nu}(x)$ decreases exponentially at large $x$, one can restrict the integrations in Eqs. (4.1)-(4.2) to values $v$ and $r$ such that $\bar{Q}_{f} r<1$. Besides, for $x \ll 1$, $\mathrm{K}_{1}(x) \sim 1 / x$ and $\mathrm{K}_{0}(x) \sim \ln (1 / x)$, so the Bessel functions can be approximated as

$$
\mathrm{K}_{0}(x) \sim \Theta(1-x), \quad \mathrm{K}_{1}(x) \sim \frac{1}{x} \Theta(1-x)
$$

where we ignore the overall normalization as well as the logarithmic singularity of $\mathrm{K}_{0}$ as $x \rightarrow 0$ (the latter is innocuous within the relevant convolutions). Another simplification

arises when performing the integral over $v$ : the constraint $v(1-v) Q^{2}<1$ together with the fact that $v(1-v) \leq 1 / 4$ for $0 \leq v \leq 1$ makes it natural to distinguish between

(a) symmetric configurations, for which $Q^{2} r^{2}<4$ ("small dipoles"), and 
(b) aligned jet configurations, for which $4<Q^{2} r^{2}$ ("large dipoles").

In the first case, there is no restriction on the $v$ integral, which is then dominated by symmetric values $v \sim 1 / 2$. In the second case, the dominant contributions correspond to the situation in which one of the two dipoles carries most of the total longitudinal momentum, that is, $v$ is either close to zero, or close to one, which allows us to further simplify the respective integrand. As a result of such simplifications, the convolutions involving the photon wavefunctions can be finally estimated as shown in Eqs. (4.9)-(4.10).

\section{B The diffractive cross-section near $\beta=1$}

In this Appendix we present the result for the differential diffractive cross-section per unit rapidity for $\beta \simeq 1$ as obtained when relaxing the leading-logarithmic approximation in $\ln (1 / \beta)$. As compared to the formulæ in the main text, this quantity represents the integrand which would give the elastic piece in the r.h.s. of Eqs. (2.1) if that quantity was computed beyond the leading-log approximation w.r.t. $\ln (1 / \beta)$. Notice that, within the limits of that approximation, the elastic scattering implies $\beta=1$, hence the corresponding differential distribution is just a $\delta$-function $\sim \delta(\beta-1)$. But this distribution gets smeared after more accurately taking into account the kinematics for the diffractive production of the $q \bar{q}$ pair into which the virtual photon has fluctuated.

In the leading-logarithmic approximation with respect to $\ln (1 / x)$, one finds $[36,37]$

$$
\begin{array}{r}
\frac{\mathrm{d} \sigma_{\mathrm{diff}}^{q \bar{q}}}{\mathrm{~d}^{2} b \mathrm{~d} \ln (1 / \beta)}=\frac{Q^{2}}{4 \pi \beta} \sum_{f} \int \mathrm{d}^{2} \boldsymbol{r} \mathrm{d}^{2} \boldsymbol{r}^{\prime} \mathrm{d} v v(1-v) \Theta\left(\boldsymbol{\kappa}_{f}^{2}\right) \mathrm{e}^{i \boldsymbol{\kappa}_{f} \cdot\left(\boldsymbol{r}^{\prime}-\boldsymbol{r}\right)} \\
\sum_{\alpha=L, T} \Phi_{\alpha}^{f}\left(v, \boldsymbol{r}, \boldsymbol{r}^{\prime}\right)\langle T(\boldsymbol{r}, \boldsymbol{b})\rangle_{Y_{\text {gap }}}\left\langle T\left(\boldsymbol{r}^{\prime}, \boldsymbol{b}\right)\right\rangle_{Y_{\text {gap }}}
\end{array}
$$

where $\boldsymbol{\kappa}_{f}^{2}=v(1-v) M_{X}^{2}-m_{f}^{2}$. The photon wavefunctions $\Phi_{T}^{f}$ and $\Phi_{L}^{f}$ are given by

$$
\begin{array}{r}
\Phi_{T}^{f}\left(v, \boldsymbol{r}, \boldsymbol{r}^{\prime}\right)=\frac{\alpha_{\mathrm{em}} N_{c}}{2 \pi^{2}} e_{f}^{2}\left(\left(v^{2}+(1-v)^{2}\right) \bar{Q}_{f}^{2}\right. \\
\frac{\boldsymbol{r} \cdot \boldsymbol{r}^{\prime}}{r r^{\prime}} \mathrm{K}_{1}\left(\bar{Q}_{f} r\right) \mathrm{K}_{1}\left(\bar{Q}_{f} r^{\prime}\right) \\
\left.+m_{f}^{2} \mathrm{~K}_{0}\left(\bar{Q}_{f} r\right) \mathrm{K}_{0}\left(\bar{Q}_{f} r^{\prime}\right)\right)
\end{array}
$$

(we use the same notations as in Appendix A) and respectively

$$
\Phi_{L}^{f}\left(v, \boldsymbol{r}, \boldsymbol{r}^{\prime}\right)=\frac{\alpha_{\mathrm{em}} N_{c}}{2 \pi^{2}} e_{f}^{2} 4 Q^{2} v^{2}(1-v)^{2} \mathrm{~K}_{0}\left(\bar{Q}_{f} r\right) \mathrm{K}_{0}\left(\bar{Q}_{f} r^{\prime}\right)
$$

with $r=|\boldsymbol{r}|$ and $r^{\prime}=\left|\boldsymbol{r}^{\prime}\right|$. Note that these functions are such that

$$
\sum_{f} \Phi_{\alpha}^{f}(v, \boldsymbol{r}, \boldsymbol{r})=\left|\psi_{\alpha}^{\gamma}(v, r ; Q)\right|^{2}
$$

is the photon wavefunction squared discussed in Appendix A. Note furthermore that the r.h.s. of (B.1) depends upon $\beta$, via $\boldsymbol{\kappa}_{f}^{2}$. (Recall that $\beta=Q^{2} /\left(Q^{2}+M_{X}^{2}\right)$.) 
The cross-section $\left(\mathrm{d} \sigma_{\text {diff }}^{q \bar{q}} / \mathrm{d}^{2} b\right)$ is obtained by integrating (B.1) from $\ln (1 / \beta)=0$ up to $Y-Y_{\text {gap }}^{\min }$. If this upper limit is relatively large, one recovers the $q \bar{q}$ part of our expression in the main text, cf. Eq. (4.6) (with $Y_{\text {gap }}^{\text {min }}$ renoted as $Y_{\text {gap }}$, for simplicity) :

$$
\frac{\mathrm{d} \sigma_{\mathrm{diff}}^{q \bar{q}}}{\mathrm{~d}^{2} b}\left(Y, Y_{\text {gap }}, Q^{2}\right)=\int \mathrm{d}^{2} \boldsymbol{r} \int \mathrm{d} v \sum_{\alpha=L, T}\left|\psi_{\alpha}^{\gamma}(v, r ; Q)\right|^{2}\langle T(\boldsymbol{r}, \boldsymbol{b})\rangle_{Y_{\text {gap }}}^{2} .
$$

This can be seen by changing the integration variable to $\kappa_{f}^{2}$ : the typical values of $r$ and $r^{\prime}$ are set by the photon wavefunctions, and thus are of order $1 / \bar{Q}_{f}$. On the other hand, the integral over $\kappa_{f}^{2}$ fixes the difference $r-r^{\prime}$ to be of order $1 / \kappa_{f}^{\max }$, with $\kappa_{f}^{\max }$ determined by the maximal diffractive mass $M_{X}^{2}$ (the one corresponding to the minimal rapidity gap $Y_{\text {gap }}$ ). When $\kappa_{f}^{\max } \gg \bar{Q}_{f}$ (in practice, this condition amounts to $M_{X}^{2} \gg Q^{2}$ ), the difference $r-r^{\prime}$ is small as compared to both $r$ and $r^{\prime}$, so one can approximate the integral $\int \mathrm{d}^{2} \kappa_{f} e^{i \boldsymbol{\kappa}_{f} \cdot\left(\boldsymbol{r}^{\prime}-\boldsymbol{r}\right)}$ by $\delta^{(2)}\left(\boldsymbol{r}^{\prime}-\boldsymbol{r}\right)$, which then yields (B.5).

Note finally that the differential cross-section is proportional to the diffractive structure function $F_{2}^{D, 3}\left(x, Q^{2}, \beta\right)$, which is measured at HERA:

$$
F_{2}^{D, 3}\left(x, Q^{2}, \beta\right)=\frac{Q^{2} \beta}{4 \pi^{2} \alpha_{\mathrm{em}} x} \frac{\mathrm{d} \sigma_{\mathrm{diff}}^{\gamma}}{\mathrm{d} \ln (1 / \beta)} .
$$

\section{References}

[1] E. Iancu and A.H. Mueller, Nucl. Phys. A730 (2004) 494.

[2] A.H. Mueller and A.I. Shoshi, Nucl. Phys. B692 (2004) 175.

[3] E. Iancu, A.H. Mueller and S. Munier, Phys. Lett. B606 (2005) 342.

[4] E. Iancu and D.N. Triantafyllopoulos, Nucl. Phys. A756 (2005) 419.

[5] I. Balitsky, Nucl. Phys. B463 (1996) 99; Phys. Lett. B518 (2001) 235; "High-energy QCD and Wilson lines", arXiv:hep-ph/0101042.

[6] J. Jalilian-Marian, A. Kovner, A. Leonidov and H. Weigert, Nucl. Phys. B504 (1997) 415; Phys. Rev. D59 (1999) 014014; J. Jalilian-Marian, A. Kovner, H. Weigert, Phys. Rev. D59 (1999) 014015; A. Kovner, J. G. Milhano, H. Weigert, Phys. Rev. D62 (2000) 114005.

[7] H. Weigert, Nucl. Phys. A703 (2002) 823.

[8] E. Iancu, A. Leonidov, L. McLerran, Nucl. Phys. A692 (2001) 583; Phys. Lett. B510 (2001) 133; E. Ferreiro, E. Iancu, A. Leonidov and L. McLerran, Nucl. Phys. A703 (2002) 489.

[9] L. McLerran and R. Venugopalan, Phys. Rev. D49 (1994) 2233; ibid. 49 (1994) 3352; ibid. 50 (1994) 2225.

[10] Yu.V. Kovchegov, Phys. Rev. D60 (1999) 034008; ibid. D61 (1999) 074018.

[11] G.P. Salam, Nucl. Phys. B449 (1995) 589; Nucl. Phys. B461 (1996) 512; A.H. Mueller and G.P. Salam, Nucl. Phys. B475 (1996) 293. 
[12] A.H. Mueller, Nucl. Phys. B415 (1994) 373; A.H. Mueller and B. Patel, Nucl. Phys. B425 (1994) 471; A.H. Mueller, Nucl. Phys. B437 (1995) 107.

[13] L.N. Lipatov, Sov. J. Nucl. Phys. 23 (1976) 338;

E.A. Kuraev, L.N. Lipatov and V.S. Fadin, Zh. Eksp. Teor. Fiz 72, 3 (1977) (Sov. Phys. JETP 45 (1977) 199);

Ya.Ya. Balitsky and L.N. Lipatov, Sov. J. Nucl. Phys. 28 (1978) 822.

[14] E. Iancu and D.N. Triantafyllopoulos, Phys. Lett. B610 (2005) 253.

[15] A.H. Mueller, A.I. Shoshi, S.M.H. Wong, Nucl. Phys. B715 (2005) 440.

[16] E. Iancu and A.H. Mueller, Nucl. Phys. A730 (2004) 460.

[17] A. Kovner and M. Lublinsky, Phys. Rev. D71 (2005) 085004.

[18] A. Kovner and M. Lublinsky, Phys. Rev. Lett. 94 (2005) 181603.

[19] A. Kovner and M. Lublinsky, Phys. Rev. D72 (2005) 074023.

[20] Y. Hatta, E. Iancu, L. McLerran, A. Stasto, and D.N. Triantafyllopoulos, Nucl. Phys. A764 (2006) 423.

[21] C. Marquet, A.H. Mueller, A.I. Shoshi, and S.M.H. Wong Nucl. Phys. A762 (2005) 252.

[22] Y. Hatta, E. Iancu, L. McLerran, and A. Stasto, Nucl. Phys. A762 (2005) 272.

[23] I. Balitsky, Phys. Rev. D72 (2005) 074027.

[24] A. Kovner and M. Lublinsky, Nucl. Phys. A767 (2006) 171.

[25] J.-P. Blaizot, E. Iancu, K. Itakura, D.N. Triantafyllopoulos, Phys. Lett. B615 (2005) 221.

[26] E. Levin and M. Lublinsky, Nucl. Phys. A763 (2005) 172.

[27] P. Rembiesa and A.M. Stasto, Nucl. Phys. B725 (2005) 251.

[28] G. Soyez, Phys. Rev. D72 (2005) 016007.

[29] R. Enberg, K. Golec-Biernat and S. Munier, Phys. Rev. D72 (2005) 074021.

[30] E. Iancu, G. Soyez and D.N. Triantafyllopoulos, "On the Probabilistic Interpretation of the Evolution Equations with Pomeron Loops in QCD” arXiv:hep-ph/0510094.

[31] A. I. Shoshi and B.-W. Xiao, Pomeron loops in zero transverse dimensions, arXiv:hep$\mathrm{ph} / 0512206$.

[32] N. Armesto and J.G. Milhano, "On correlations and discreteness in non-linear QCD evolution", arXiv:hep-ph/0601132.

[33] S. Munier and R. Peschanski, Phys. Rev. Lett. 91 (2003) 232001; Phys. Rev. D69 (2004) 034008; ibid. D70 (2004) 077503.

[34] E. Levin and M. Wüsthoff, Phys. Rev. D50 (1994) 4306.

[35] J. Bartels and M. Wüsthoff, Z. Phys. C66 (1995) 157; ibid. 68 (1995) 121.

[36] A. Bialas and R. Peschanski, Phys. Lett. B378 (1996) 302; Phys. Lett. B387 (1996) 405;

A. Bialas, R. Peschanski and C. Royon, Phys. Rev. D57 (1998) 6899. 
[37] M. Wüsthoff, Phys. Rev. D56 (1997) 4311.

[38] W. Buchmuller, M.F. McDermott and A. Hebecker, Nucl. Phys. B487 (1997) 283; Erratum ibid. B500 (1997) 621; W. Buchmuller, T. Gehrmann and A. Hebecker Nucl. Phys. B537 (1999) 477.

[39] Yu.V. Kovchegov and L. McLerran, Phys. Rev. D 60 (1999) 054025.

[40] K. Golec-Biernat and M. Wüsthoff, Phys. Rev. D59 (1999) 014017; ibid. D60 (1999) 114023.

[41] J. Bartels, H. Jung and M. Wüsthoff, Eur. Phys. J. C11 (1999) 111.

[42] B. Z. Kopeliovich, A. Schaefer and A. V. Tarasov, Phys. Rev. D62 (2000) 0540022.

[43] E. Gotsman, E. Levin, M. Lublinsky, U. Maor and K. Tuchin, Phys. Lett. B492 (2000) 47; Nucl. Phys. A697 (2002) 521.

[44] Yu.V. Kovchegov and E. Levin, Nucl. Phys. B577 (2000) 221.

[45] Yu. V. Kovchegov, Phys. Rev. D64 (2001) 114016.

[46] A. Kovner and U. Wiedemann, Phys. Rev. D64 (2001) 114002.

[47] J. Bartels, K. Golec-Biernat and H. Kowalski, Phys. Rev. D66 (2002) 014001.

[48] E. Gotsman, E. Levin, M. Lublinsky and U. Maor, Eur. Phys. J. C27 (2003) 411.

[49] E. Iancu, K. Itakura and S. Munier, Phys. Lett. B590 (2004) 199.

[50] S. Munier and A. Shoshi, Phys. Rev. D69 (2004) 074022.

[51] J.R. Forshaw, R. Sandapen and G. Shaw, Phys. Lett. B594 (2004) 283; J.R. Forshaw and G. Shaw, JHEP 0412 (2004) 052.

[52] C. Marquet, Nucl. Phys. B705 (2005) 319.

[53] K. Golec-Biernat and C. Marquet, Phys. Rev. D71 (2005) 114005.

[54] M.S. Kugeratski, V.P. Goncalves and F.S. Navarra, "Saturation in diffractive deep inelastic eA scattering", arXiv:hep-ph/0511224.

[55] M. Wüsthoff and A. Martin, J. Phys G25 (1999) R309.

[56] A. Hebecker, Phys. Rept. 331 (2000) 1.

[57] G. Ingelman, "Hard diffraction - 20 years later", arXiv:hep-ph/0512146.

[58] M.L. Good and W.D. Walker, Phys. Rev. 120 (1960) 1857.

[59] J. Pumplin, Phys. Rev. D8 (1973) 2899; H. I. Miettinen and J. Pumplin, Phys. Rev. D18 (1978) 1696.

[60] A.M. Stasto, K. Golec-Biernat and J. Kwiecinski, Phys. Rev. Lett. 86 (2001) 596.

[61] E. Iancu, K. Itakura, and L. McLerran, Nucl. Phys. A708 (2002) 327.

[62] A.H. Mueller and D.N. Triantafyllopoulos, Nucl. Phys. B640 (2002) 331.

[63] D.N. Triantafyllopoulos, Nucl. Phys. B648 (2003) 293. 
[64] A. Kovner and U.A. Wiedemann, Phys. Rev. D66 (2002) 034031; ibidem 051502.

[65] E. Ferreiro, E. Iancu, K. Itakura, and L. McLerran, Nucl. Phys. A710 (2002) 373.

[66] E. Brunet and B. Derrida, Phys. Rev. E56 (1997) 2597; Comp. Phys. Comm. 121-122 (1999) 376; J. Stat. Phys. 103 (2001) 269.

[67] E. Brunet, B. Derrida, A. H. Mueller and S. Munier, "A phenomenological theory giving the full statistics of the position of fluctuating pulled fronts," arXiv:cond-mat/0512021.

[68] L.V. Gribov, E.M. Levin, and M.G. Ryskin, Phys. Rept. 100 (1983) 1.

[69] A.H. Mueller and J. Qiu, Nucl. Phys. B268 (1986) 427.

[70] A. H. Mueller, Nucl. Phys. B335 (1990) 115.

[71] N.N. Nikolaev and B.G. Zakharov, Z. Phys. C49 (1991) 607, ibid. C53 (1992) 331.

[72] E. Levin and M. Lublinsky, Phys. Lett. B607 (2005) 131.

[73] E. Levin and M. Lublinsky, Nucl. Phys. A730 (2004) 191.

[74] M. Braun, Phys. Lett. B483 (2000) 115; "Conformal invariant equations for nucleus-nucleus scattering in perturbative QCD with $N_{c} \rightarrow \infty$ ”, arXiv:hep-ph/0504002.

[75] M. Braun and G.P. Vacca, Eur. Phys. J. C6 (1999) 147; M. Braun, Phys. Lett. B483 (2000) 115.

[76] M. Hentschinski, H. Weigert and A. Schafer, "Extension of the color glass condensate approach to diffractive reactions", arXiv:hep-ph/0509272.

[77] Z. Chen and A. H. Mueller, Nucl. Phys. B451 (1995) 579.

[78] Yu. V. Kovchegov and A. H. Mueller, Nucl. Phys. B529 (1998) 451.

[79] E. Iancu, K. Itakura, and L. McLerran, Nucl. Phys. A724 (2003) 181.

[80] N. Armesto and M. Braun, Eur. Phys. J. C20 (2001) 517; ibid. C22 (2001) 351.

[81] K. Golec-Biernat, L. Motyka and A.M. Staśto, Phys. Rev. D65 (2002) 074037.

[82] E. Levin and M. Lublinsky, Phys. Lett. B521 (2001) 233; Eur. Phys. J. C22 (2002) 647; M. Lublinsky, Eur. Phys. J. C21 (2001) 513.

[83] K. Rummukainen and H. Weigert, Nucl. Phys. A739 (2004) 183.

[84] C. Marquet, R. Peschanski and G. Soyez, Nucl. Phys. A756 (2005) 399; C. Marquet and G. Soyez, Nucl. Phys. A760 (2005) 208.

[85] D. E. Kharzeev, E. Levin and L. McLerran, Phys. Lett. B561 (2003) 93.

[86] E. Iancu, K. Itakura and D.N. Triantafyllopoulos, Nucl. Phys. A742 (2004) 182.

[87] E. Moro, Phys. Rev. E70 (2004) 045102(R).

[88] C. Marquet, R. Peschanski and G. Soyez, "Consequences of strong fluctuations on highenergy QCD evolution", arXiv:hep-ph/0512186.

[89] For a recent review, see W. Van Saarloos, Phys. Rep. 386 (2003) 29. 